\title{
Adaptive Control in the Presence of Input \\ Constraints
}

\author{
by \\ Steingrímur Páll Kárason
}

Submitted to the Department of Mechanical Engineering in partial fulfillment of the requirements for the degree of

Master of Science in Mechanical Engineering

at the

MASSACHUSETTS INSTITUTE OF TECHNOLOGY

$$
\text { May } 1993
$$

(C) Massachusetts Institute of Technology 1993. All rights reserved.

Author ..................

\section{Signature redacted}

Department of Mechanical Engineering

May 18, 1993

Certified by......

\section{Signature redacted}

\author{
Anuradha M. Annaswamy \\ Assistant Professor \\ Thesis Supervisor
}

\section{Signature redacted}

Accepted by ...................

Professor A. A. Sonin Chairman, Departmental Committee on Graduate Students ARCHIVES 


\title{
Adaptive Control in the Presence of Input Constraints
}

by

\author{
Steingrímur Páll Kárason \\ Submitted to the Department of Mechanical Engineering \\ on May 18, 1993, in partial fulfillment of the \\ requirements for the degree of \\ Master of Science in Mechanical Engineering
}

\begin{abstract}
This thesis deals with the problem of adaptively controlling a linear time-invariant plant in the presence of constraints on the input amplitude. A new algorithm is introduced for continuous-time plants and it is shown that it leads to bounded solutions when the initial conditions of the adaptive system lie within a compact set. A similar condition is also put forward for discrete-time plants which ensures bounded trajectories. In both cases the results are valid for open-loop stable as well as unstable plants but are restricted to minimum phase plants. The results are applied to the problem of grasping and manipulating a compliant object using compliant fingerpads.
\end{abstract}

Thesis Supervisor: Anuradha M. Annaswamy

Title: Assistant Professor 


\section{Contents}

$\begin{array}{lll}1 & \text { Introduction } & 7\end{array}$

1. Constraints in Automatic Control . . . . . . . . . . . . . . . 8

1.1 Stability of Input-Constrained Systems . . . . . . . . . . . . 10

2. Adaptive Control with Limits on the Input Amplitude . . . . . . . . 11

3. New Results . . . . . . . . . . . . . . . . . . 12

4. Application of Constrained Adaptive Control . . . . . . . . . . . . . 12

2 Overview 15

1. Constraints in Automatic Control . . . . . . . . . . . . . . . . 15

2. Functional or State Constraints . . . . . . . . . . . . . . . . 16

3. Stability of Input Constrained Systems . . . . . . . . . . . . . 16

4. Adaptive Control with Limits on Input Amplitude . . . . . . . . . . . 21

4.1 Stability of Adaptive Systems with a Saturated Input . . . . . 23

4.2 Conclusion ....................... 26

5. Papers Published on Adaptive Control in the Presence of Constraints 26

3 Adaptive Control of Continuous-Time Systems $\quad 27$

1. A First-Order Plant . . . . . . . . . . . . . . . . . 27

2. State Variables Accessible . . . . . . . . . . . . . . . . . 30

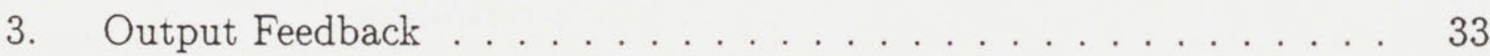

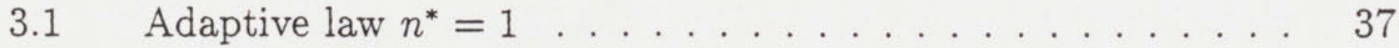

3.2 Adaptive Law, $n^{*} \geq 2$. . . . . . . . . . . . 38

3.3 Boundedness of Signals . . . . . . . . . . . . . . . . . 39 
4. Robustness .......................... 41

4.1 Disturbances ......................... 41

4.2 Modification of Adaptive Law . . . . . . . . . . . . . . . 42

4.3 Stability ....................... . . . . 44

5. Simulations .......................... 45

$5.1 \quad$ 1st Order System . . . . . . . . . . . . . 45

5.2 Output Feedback, Relative degree 2 . . . . . . . . . . . 45

4 Adaptive Control of Discrete-Time Systems $\quad 55$

1. Model Reference Adaptive Control of a First Order Plant . . . . . . . 55

1.1 Stability .......................... 57

2. One-Step-Ahead Adaptive Control of an $n$th Order Plant . . . . . . . 58

2.1 Stability ........................ 60

3. Control in the presence of Bounded Disturbances . . . . . . . . . 62

4. Simulations .......................... 64

4.1 Model Reference Control of a 1st Order System . . . . . . . . 64

4.2 One-Step-Ahead Control of a 2nd Order System . . . . . . . 65

5 Application of Bounded Input Adaptive Control $\quad 75$

1. Compliant Fingerpads . . . . . . . . . . . . . . . . 76

1.1 Computational Theory of Haptics . . . . . . . . . . . . . . 77

2. Dynamic Model . . . . . . . . . . . . . . . 78

2.1 Constraints ..................... 81

2.2 Satisfaction of Constraints . . . . . . . . . . . . . 81

3. Control Design ......................... 83

3.1 An Adaptive Controller . . . . . . . . . . . . . 83

$\begin{array}{lll}6 & \text { Summary and Conclusions } & 87\end{array}$

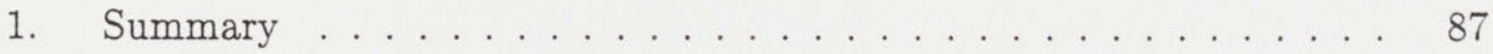

2. Conclusions ......................... 87

$\begin{array}{ll}\text { A Proofs of Theorems in Chapter } 3 & 89\end{array}$ 
1. Proof of Theorem 3.1 . . . . . . . . . . . . . . 89

2. Proof of Theorem 3.2 . . . . . . . . . . . . . . . . 91

3. Proof of Theorem $3.3 \ldots \ldots \ldots$. . . . . . . . . . . . 94

4. Proof of Theorem 3.4 . . . . . . . . . . . . . . . 96

B Proofs for Theorems in Chapter 4 100

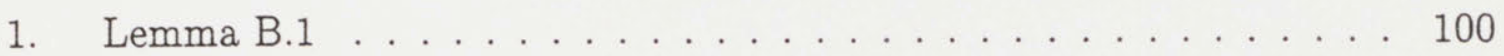

2. Proof of Theorem 4.1 .................. . . 101

3. Proof of Theorem 4.2 . . . . . . . . . . . . . . 104

4. Proof of Theorem $4.3 \ldots \ldots \ldots$. . . . . . . . . . . 107 


\section{List of Figures}

2-1 System Block-Diagram . . . . . . . . . . . . . . 17

3-1 System Block-Diagram with Input-Disturbances. . . . . . . . . . . 42

3-2 Case (a) for Adaptive Control of a 1st-order Continuous-Time Plant. 47

3-3 Case (b) for Adaptive Control of a 1st-order Continuous-Time Plant. 48

3-4 Case (c) for Adaptive Control of a 1st-order Continuous-Time Plant. 49

3-5 Case (d) for Adaptive Control of a 1st-order Continuous-Time Plant. 50

3-6 Case (a) for Adaptive Control of a 2nd-order Continuous-Time Plant. 51

3-7 Case (b) for Adaptive Control of a 2nd-order Continuous-Time Plant. 52

3-8 Case (c) for Adaptive Control of a 2nd-order Continuous-Time Plant. 53

3-9 Case (d) for Adaptive Control of a 2nd-order Continuous-Time Plant. 54

4-1 Case (a) for Adaptive Control of a 1st-order Discrete-Time Plant. . . 67

4-2 Case (b) for Adaptive Control of a 1st-order Discrete-Time Plant. . . 68

4-3 Case (c) for Adaptive Control of a 1st-order Discrete-Time Plant. . . 69

4-4 Case (d) for Adaptive Control of a 1st-order Discrete-Time Plant. . . 70

4-5 Case (a) for One-Step-Ahead Adaptive Control of a Discrete-Time Plant. 71

4-6 Case (b) for One-Step-Ahead Adaptive Control of a Discrete-Time Plant. 72

4-7 Case (c) for One-Step-Ahead Adaptive Control of a Discrete-Time Plant. 73

4-8 Case (d) for One-Step-Ahead Adaptive Control of a Discrete-Time Plant. 74

5-1 A schematic diagram of the dynamic model. . . . . . . . . . . 86 


\section{Chapter 1}

\section{Introduction}

One of the major problems that arises while controlling dynamic systems is the tradeoff between reaching maximum performance and robustness while using only practically realizable control inputs. Physical limitations dictate that hard limits be imposed on the magnitude to avoid damage to or deterioration of the process. Therefore, any active input that is determined on-line should meet the desired control objectives while remaining within certain limits. Also, from an analytical point of view, there may be a need to maintain the process near the desired operating point in order to make a linear control design adequate. This may in turn force the input magnitude to lie within certain bounds.

For example consider two fingers pinch grasping an object and moving it between two points. If both the fingers and the object are compliant then the dynamic characteristics of either one (such as stiffness and damping) influence the compressive force exerted by the fingers on the object. Also, the size of the contact area changes with the applied compressive force which in turn may affect the stability of the grasp. If the object is not to get dropped during manipulation the contact forces must be in compression at all times and the control signals must therefore be constrained to only those that produce compression between the object and both the fingers. Typically, the dynamic characteristics mentioned above are unknown and will vary with different grasp positions. This in turn means that some kind of adaptive control will be needed to ensure successful manipulation. Hence, ensuring that the control input does not 
exceed these bounds while simultaneously realizing the performance objectives is a very important problem.

Over the years, adaptive control methods have been developed to control systems that are poorly known. In particular, parametric uncertainties in linear dynamic systems have been effectively dealt with using adaptive control. Stability as well as robustness issues of adaptively controlling linear time-invariant plants with unknown parameters are well understood and documented in several textbooks and papers $[1,2,3]$. The focus in all these cases has been on determining the least restrictive conditions on the plant under which a control input can be realized to meet various control objectives. There was however no restriction on the magnitude of the control input, which commonly is the case as described in the last paragraph. In this thesis this problem of adaptive control with constrained input will be addressed and new methods and algorithms will be proposed to meet the constraints as well as the control objectives.

Chapter 2 describes how limits on the input amplitude affect the performance and stability of linear systems with emphasis on adaptive control. A review of the existing literature on adaptive control with input constraints is also given.

In Chapter 3 and 4, some new results on the problems described in chapter 2 are stated in Theorems 3.1-3.4 in Chapter 3, and 4.1-4.3 in Chapter 2. There modified control schemes and the stability of the overall system is established and results supported by simulations studies.

Chapter 4 describes an application of the results in Chapter 3. A simplified model of compliant fingerpads is analyzed and it is shown how the control of this model can be represented in the framework of constrained adaptive control.

\section{Constraints in Automatic Control}

Control with constraints on the available control amplitude commonly occurs and is usually described as a linear plant with a nonlinear element describing the constraint, between the controller and the plant. Classical control theory does not have any 
general tools or methods for the treatment of input non-linearities that are readily applicable to input saturation. This is especially true in the case of unstable systems where stability becomes naturally a primary concern if only a limited range of control values is allowed for whatever reason. Then the instability demanding large stabilizing inputs conflicts with the bounds on the input and a well founded stability theory is needed for the control design.

There has been considerable work done in the area of nonlinear systems stability $[4,5,6]$. While they are applicable and quite useful for specific classes of nonlinear systems they are inadequate for our purpose of determining conditions under which a linear time-invariant plant can be controlled inside a frame of input constraints. For instance, Describing Function Analysis consists of replacing a nonlinear element within the system with a specifically constructed linear element and then analyzing the resulting system. It is an approximate method and subject to some assumptions about the system and the nonlinearity and is mostly useful to predict the onset of limit-cycles and related phenomena. One can easily construct a description function for a hard-saturation but the assumptions made are too restrictive to be applicable to adaptive systems with any certainty. The Luré Problem refers to the control of a system whose forward path is linear and has a sector nonlinearity in the feedback path. Although hard saturation is, strictly speaking, not a sector nonlinearity, some of the many solutions developed for the Lure problem have included treatment of hard-saturation and time-varying nonlinearity $[7,5,4]$ but they generally do not explicitly address unstable systems. Popov's Criterion is a solution to a specific case of Luré Problem and gives a condition for systems, which include sector nonlinearities, similar to what Nyquist's criterion gives for linear systems, leading to a condition in the frequency domain. Since it does not treat non-autonomous systems, it is not of use here. The Circle Criterion $[8,9]$ and $[7,10]$ is a more generalized version of Nyquist's Criterion than Popov's Criterion. It can be extended to non-autonomous systems, but like all the methods above the results are interpreted in the frequency domain and hence the result is obtained in global terms. That is, the question if the system can be guaranteed to be globally stable or not is answered. We will show 
that, this approach is inadequate for unstable systems with hard-saturation as they can only be locally stable. None of these methods directly addresses the stability of non-autonomous systems with time-varying linear feedback and hard saturation in the feed-forward path, which is the case for adaptive control with saturation. Finally, one important fact from the viewpoint of design is to have conditions that show what factors affect stability and in what way they do so.

\subsection{Stability of Input-Constrained Systems}

As the main focus of this thesis is on the stability properties of systems with input saturation some of the general stability properties of such systems are stated below. The system considered consists of a linear time-invariant, single-input plant and a controller when there is a hard limit on the amplitude of the command signal going from the control to the plant, (see Fig. 1.) Although this is a fairly general, structure it has some important properties, which are simply stated here but detailed and explained further in chapter 2 .

\section{Open-Loop Stable Plants}

- In a system with a Bounded-Input, Bounded-Output stable (BIBO) plant, the plant can not be made unstable by any controller when there is a limit on the control signal. In such a case the input is always bounded and therefore the plant variables remain bounded.

- Then the stability of the controller is all that remains to be shown to ensure the stability of the overall system

\section{Open-Loop Unstable Plants}

- If the plant is strictly unstable then there exists an initial condition for the plant so that the system is unstable for all subsequent control inputs. This fact becomes obvious if the plant is considered in state description of Jordan form. Therefore the system can only be shown to be stable when the initial conditions 
are within a compact set, which forms a stabilizable region in the state-space, i.e. locally stable.

- It can also be shown that there exist a bounded reference input which can drive the plant out of the stabilizable region for a fairly general class of controllers. Therefore one can not show stability for all finite reference trajectories but only for those that remain within some bounds. Hence, one of the quantities that need to be determined for stability are these bounds.

- If an adaptive controller is used in addition to the above mentioned bounds, the initial condition of the control parameter must be bounded as well, since in that case the control parameter is also a part of the system state. Initial values outside these bounds could drive the plant into instability.

Having these facts in mind, it is clear that stability for an adaptive system with hard saturation is local and the condition must depend in some way on all the factors detailed above.

\section{Adaptive Control with Limits on the Input Amplitude}

Adaptive control is no exception from other control methods in that large input amplitudes must frequently be constrained, although in classical adaptive control theory it is generally assumed that unlimited amplitude is available. Still, if not intentionally avoided, it typically results in high control signals which cannot be implemented by actuators in practice. One possible way to avoid this is to penalize the control input amplitude in some way and methods for that have been developed [3]. Penalizing the control amplitude results in lower amplitudes but does not give any insight into, whether the system will be stable or not. For direct adaptive control of stable discrete-time plants the effect of saturation is not very serious if good care is taken, as the adaptive law can easily be made stable independent of any saturation effect, 
provided that the saturation bounds are known [3]. This is not true for continuoustime systems and algorithms will not work correctly unmodified. A basic structure for modifying the continuous-time adaptive law for effects of saturation limits was proposed by Monopoli in 1975 [11] but no stability proof was provided in this paper. Much of this thesis will be on showing how these algorithms need to be modified.

\section{New Results}

The new results that this thesis contains are in the area of adaptive control of linear time-invariant, SISO plants with input constraints, and are in two parts.

New algorithms for continuous-time systems which ensure that parameters are estimated correctly with saturation, are put forward for the most common cases. That includes, for first-order plants, states-accessible plants, relative-degree equal one, and plants of relative degree two and greater. In all cases the results are restricted to minimum-phase plants as when the control is unconstrained.

Stability theorems are proved for all the algorithms above and also for two prototype cases of discrete-time plants, i.e. for model reference adaptive control of first-order plants and for one-step-ahead direct adaptive control for plants of arbitrary known order.

\section{Application of Constrained Adaptive Control}

As stated earlier in this chapter, manipulation problems with pinch finger grasp typically lead to control with input constraints. Then the mathematical model is based on the assumption that friction between the two surfaces in each contact prevents slip. This assumption can only be true if the contact force is compressive and results in sufficient pressure in the contact area to counteract tangential forces. If the fingerpads and the object are assumed to be compliant the complexity increases since the interaction is dynamic and possibly unknown. The reason for exploring this problem 
is to understand what principles lie behind successful completion of robotic tasks in manufacturing which involve mechanical interactions between the robot and its environment. Examples of such tasks are drilling, grinding, inserting a peg-in-a-hole, and assembly operations. Also, for general purpose robots that need to explore and manipulate objects in their environment, the presence of compliance in the end-effector and explicit modeling of it is desirable for many reasons. The ones that come readily to mind are:

- Compliant fingers an object will generally have a finite area of contact, in contrast to point contact as is the case when the fingers and the object are assumed to be rigid. The introduction of a finite area of contact gives more stable grasp for fewer fingers as then additional resisting and stabilizing torsional torque appears, which resists rotation around normal to the contact area. The effects of this additional torque can well be seen from the fact that the minimum number of finger contacts to ensure a stable grasp decreases from two to three when the finite are of contact is introduced.

- The stresses resulting in the contact region will be much less in the compliant case as the total force is distributed over the area of contact and not concentrated as in the case of a point contact. This leads to increased dexterity as more fragile objects can then be manipulated.

- Exact shape of the object in the contact region will be less important for stability in the compliant case and not as crucial as in the rigid case.

- The explicit modeling of the compliance in the finger and the object will lead to dynamic models of higher order, which to some extent provides increased maneuverability as then the control bandwidth can possibly be increased.

Often, as in the case of a general purpose robot, the objects encountered can possess a wide range of dynamic characteristics. This implies that the grasping and manipulation tasks have to be performed even when the dynamics of the object in 
question is unknown. In such cases, the control forces on the fingerpads need to be generated using an adaptive rather than a fixed controller.

The general problem of contact between two deformable bodies leads to a spatiotemporal analysis. Here, the problem will be simplified by adopting single degree-offreedom lumped parameter model, thus reducing the variables to be purely temporal. The fingers as well as the object are assumed to deform only in one direction. The aim will be able to hold a compliant object with unknown dynamic parameters in a two-finger pinch grasp and move it along any desired path in a three-dimensional, gravity environment, while ensuring that the object does not slip or get crushed.

Because the system essentially describes a motion in free air, the resulting model is unstable, having a double integrator. This is the problem that motivated the work on the control algorithms and stability theorems in this thesis. The results obtained are however applicable to a more general class of problems and form the main subject. 


\section{Chapter 2}

\section{Overview}

\section{Constraints in Automatic Control}

Control amplitude ${ }^{1}$ saturation is often the dominant nonlinearity in control design although there is very little formal theory that addresses it [14]. In the literature, the main emphasis has been on providing schemes that preserve linear behavior under saturation, if possible, and otherwise provide graceful degradation of system performance under saturation. Several ad hoc schemes have been proposed $[14,15,12,16]$, along with others which are analytically more rigorous $[17,18]$. The former schemes are, first and foremost, intended to prevent integrator windup resulting from saturation, but [17] is concentrated on preserving the properties of a linear time-invariant MIMO control design. None of the above schemes is much concerned with the domain of stability for unstable plants except for [18], which discusses the problem but does not give any explicit results on stability. The size of domain of stability is treated for systems with linear feedback in $[5,19,20]$, where the approach is to maximize the domain of stability using numerical search methods.

\footnotetext{
${ }^{1}$ Control input is most often considered to be limited only in the amplitude but a more realistic approach would be to also include a limit on the rate of the control input $[12,13]$. In this thesis, only amplitude constraints will be treated.
} 


\section{Functional or State Constraints}

Input constraints have several implications on the dynamic model and the control design. For instance, they may imply that the mathematical model used is only valid for a certain region of the state space. The region may be where linearity is preserved or where some functioning constraint must be satisfied. The region will be described by some constraint function of the state variables. When carrying out the control design, these constraints may limit the possible reference inputs as well as how the feedback signal is used in the control signal. The combined effect of these is then limited by intentionally restricting the control amplitude to a certain range. Making some assumptions on the frequency content of signals involved and using the limits obtained from the transfer function from the input to the output variable describing the constraint, the limits on the input can be projected to a range in the constraint variable.

\section{Stability of Input Constrained Systems}

Now some general stability properties of systems which consist of a single-input, linear time-invariant plant and a controller with a hard limit on the amplitude of the command signal going from the controller to the plant (see Fig. 2-1). Some of the results are independent of the structure of the controller, but in each case where that is not true the assumed structure will be detailed with the result.

\section{Stable Plants}

A linear time-invariant (LTI) plant which has a hard limit on the control input and is itself strictly open-loop stable, will always remain stable as in the linear case stability and bounded-input bounded output (BIBO) stability are equivalent [1]. The stability of the controller is then the only thing that needs to been ensured. If the controller has a integrator (e.g. a PID controller) then special care has to be taken and the danger of instability is a commonly known problem usually called Integrator windup. 


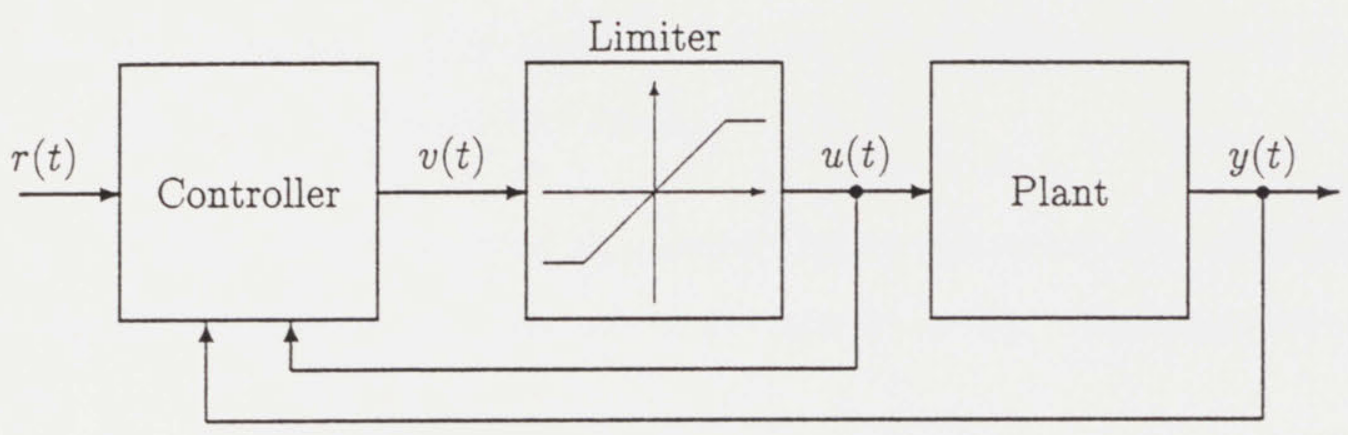

Figure 2-1: System Block-Diagram

The system that this section is devoted to investigate. The figure defines variables $r(t)$ as the reference input, $v(t)$ the control signal before saturation, $u(t)$ the control signal after saturation and $y(t)$ as the plant output

The results of $[15,14,16]$ suggest control schemes which can overcome this problem.

\section{Plants with multiple integrators}

If the plant has in it a higher order integrators but is otherwise stable, the problem of stability becomes more complicated. However results for continuous-time plants exist that give globally stabilizing controllers with bounded control amplitude, using nonlinear feedback [21]. It can further be shown that a saturating linear time-invariant feedback control, cannot possibly globally stabilize a plant with integrators of order greater than or equal to $3[22]$.

\section{Unstable Plants}

If the plant is strictly unstable, i.e. having at least one unstable pole, some important facts can be stated about the stability of the system. A clear statement of these facts cannot been found in the literature. They will therefore be stated and proved here as Lemma 1 and 2. The first result is independent of what controller is used so the controller generating the signal $u(t)$ is left unspecified. 
Let a linear, time-invariant plant have a state-space description given by

$$
\dot{x}(t)=\Lambda x(t)+b u(t) \quad|u(t)| \leq u_{\max } \forall t \geq t_{0}
$$

and assume that it is of Jordan form, with a diagonal matrix $\Lambda \in \mathbb{R}^{n \times n}$. The following lemma states that if the system in Eq. (2.1) is unstable it cannot be globally stabilized using any bounded control input.

Lemma 2.1 There exist a constant $x_{\max }$ and an initial condition $x\left(t_{0}\right)$, both of which are finite, with $\left\|x\left(t_{0}\right)\right\| \geq x_{\max }$ for which the system in Eq. (2.1) has unbounded solutions.

\section{Proof:}

Let $\lambda_{j}$ be the eigenvalue of $\Lambda$ for $j=1,2, \ldots n$, and $\lambda_{i}$ be such that it is real and

$$
\lambda_{i} \geq \operatorname{Re}\left(\lambda_{j}\right) \forall j=1,2, \ldots n
$$

Since $\Lambda$ is in a diagonal form, the equation corresponding to the eigenvalue $\lambda_{i}$ can be written as

$$
\dot{x}_{i}(t)=\lambda_{i} x_{i}(t)+b_{i} u(t) .
$$

Since $\Lambda$ is unstable, $\lambda_{i}>0$. It follows that

$$
\begin{aligned}
\frac{1}{2} \frac{d}{d t} x_{i}^{2}(t) & =\lambda_{i} x_{i}^{2}(t)+x_{i}(t) b_{i} u(t) \\
& \geq \lambda_{i}\left|x_{i}(t)\right|^{2}-\left|x_{i}(t)\right|\left|b_{i}\right| u_{\max } \\
& >0 \quad \forall\left|x_{i}(t)\right| \geq x_{\max }
\end{aligned}
$$

where

$$
x_{\max } \triangleq \frac{\left|b_{i}\right| u_{\max }}{\lambda_{i}}
$$

If $\lambda_{i}$ is complex, then the complex conjugate $\lambda_{i}^{*}$ is also an eigenvalue of $\Lambda$. Using 
the unitary state transform

$$
T \triangleq=\frac{1}{\sqrt{(2)}}\left[\begin{array}{cc}
1 & -j \\
1 & j
\end{array}\right]
$$

in defining

$$
\rho(t) \triangleq T\left[\begin{array}{c}
x_{i}(t) \\
x_{i+1}(t)
\end{array}\right] \text { and } \beta \triangleq T^{-1}\left[\begin{array}{c}
b_{i} \\
b_{i+1}
\end{array}\right] .
$$

Then the state-space spanned by the eigenvectors corresponding to these two eigenvalues satisfy the equation

$$
\dot{\rho}(t)=\mu_{i} I \rho+\omega_{i} J \rho+\beta u(t) \quad J \triangleq\left[\begin{array}{cc}
0 & 1 \\
-1 & 0
\end{array}\right],
$$

$\rho$ and $\beta$ are real, $I$ is the identy matrix and $\mu_{i}$ and $\omega_{i}$ denote, respectively, the real and the imaginary parts of $\lambda_{i}$. This gives the relation

$$
\begin{aligned}
\frac{1}{2} \frac{d}{d t} \rho_{i}^{T}(t) \rho_{i}(t)=\rho_{i}^{T}(t) \dot{\rho}_{i}(t) & =\mu_{i}\left\|\rho_{i}(t)\right\|^{2}+\rho_{i}^{T}(t) \beta u(t) \\
& \geq \mu_{i}\|\rho(t)\|^{2}-\|\rho(t)\|\|\beta\| u_{\max } \\
& >0 \quad \forall\|\rho(t)\| \geq x_{\max }
\end{aligned}
$$

where

$$
x_{\max } \triangleq \frac{\|\beta\| u_{\max }}{\mu_{i}} .
$$

From Eq. (2.4) and (2.5) it is notes that a constant $x_{\max }$ exists satisfying the conditions stated in Lemma 2.1 and hence the proof.

Now it will be shown that a bounded reference input, $r(t)$ can be found for which a feedback controller destabilizes the system defined in Fig. (2-1) and Eq. (2.1)

Let the feedback controller be described by

$$
v(t)=f(x, t)+f_{0}(x, t) r(t)
$$


where

$$
\sup _{\|x(t)\| \leq x_{\max }}\left[\frac{f(x, t)}{f_{0}(x, t)}\right] \triangleq R(t) \in \mathcal{L}^{\infty}
$$

and $f$ and $f_{0}$ are such that condition giving existence of solutions are satisfied. Further assume that the system is controllable for all times. Then we have the following lemma.

Lemma 2.2 There exists a bounded reference trajectory that destabilizes the plant in Eq. (2.1) with the controller structure in Eq. (2.7) satisfying Eq. (2.8).

Proof: As in the proof of Lemma 2.1, assume that $\lambda_{i}$, defined in Eq. (2.2) is real. If

$$
r(t)=\frac{1}{f_{0}(x, t)}\left[-f(x, t)+\varepsilon_{1} \frac{1}{b_{i}}\right] \quad \varepsilon_{1}>0
$$

then the control signals defined in Fig. 2-1 will be, $u=v=\varepsilon_{1}$ for sufficiently small $\varepsilon_{1}$. Also assume without loss of generality that $x\left(t_{0}\right) \geq 0$. This results, for this case, in

$$
\dot{x}_{i}(t)=\lambda_{i} x_{i}+\varepsilon_{1}>0 \forall t \geq t_{0}
$$

or $x_{i}(t) \rightarrow \infty$ as $t \rightarrow \infty$. Hence, any reference input of the form in Eq. (2.9) drives the system into the unstable region. It is further noted from Eq. (2.8) that $r(t)$ is bounded.

- A similar proof can be given, even if $\lambda_{i}$ is complex.

It is worth noting that a special case of the controller in Eq. (2.7) is the linear feedback controller

$$
v(t)=f(t)^{T} x(t)+f_{0}(t) r(t)
$$

which satisfies Eq. (2.8) if $f(t)$ is bounded and $f_{0}(t) \neq 0$.

Both Lemma 1 and 2 hold for discrete-time systems as well. The proofs are almost identical and are therefore omitted. For sake of completeness the discrete- time form of Lemma 2.1 will now be stated.

Lemma 2.3 (Discrete-time system) If the discrete-time system

$$
\varphi(t+1)=\Lambda_{d} \varphi(t)+b_{d} u(t) \quad|u(t)| \leq u_{\max }
$$


is unstable, with $\lambda_{d_{i}}$ defined as the eigenvalue of $\Lambda_{d} \in \mathbb{R}_{n \times n}$ with the largest modulus, $\left|\lambda_{d_{i}}\right|$. Also $b_{d_{i}}$ defined as the entry in $b_{d}$ corresponding to $\lambda_{d_{i}}$. Then there exist a constant

$$
\varphi_{\max } \triangleq \frac{\left|b_{d_{i}}\right| u_{\max }}{\left|\lambda_{d_{i}}\right|-1}
$$

and an initial condition $\varphi\left(t_{0}\right)$, both of which are finite, with $\left\|\varphi\left(t_{0}\right)\right\| \geq \varphi_{\max }$ for which the system has unbounded solutions.

\section{Adaptive Control with Limits on Input Am- plitude}

Most adaptive control algorithms that have been developed so far are for plants with a known structure but poorly known parameters. For linear plants this typically means that the order, and the relative order of the plant's transfer function are known but its coefficients are unknown. Further a distinction is made between direct and indirect adaptive control. Indirect control refers to that an intermediate step is taken, in estimating explicitly the plant parameters to be used to calculate the control parameters, whereas in direct adaptive control this step is omitted and the control parameters are estimated directly. Although both these approaches are equally valid, only the case of direct adaptive control will be treated in this thesis.

In direct adaptive control, the parameters of the controller are adapted over time, in principle, based on a comparison of the expected and the resulting plant output. The rule for the adjustment of the parameter is expressed as an adaptive law, usually in the form of a differential equation for continuous-time systems and a difference equation for discrete-time systems. The state of the overall system will therefore contain the time-varying parameters of the controller in addition to the plant and other controller states. The system state space will then necessarily be composed of linear and nonlinear subsystems which makes the overall system nonlinear and time-varying. For continuous systems the controller and the adaptive law take the 
form

$$
\begin{aligned}
v(t) & =\theta_{1}^{T}(t) x(t)+\theta_{r}(t) r(t) \\
& =\theta^{T}(t) \omega(t) \\
u(t) & =\left\{\begin{array}{ccc}
v(t) & \text { if } & |v(t)| \leq u_{\max } \\
u_{\max } \operatorname{sgn}(v(t)) & \text { if } & |v(t)|>u_{\max }
\end{array}\right. \\
\dot{\theta}(t) & =f_{c}(\omega, \theta, t)
\end{aligned}
$$

where $x(t)$ is the system state or the output from a state estimator,

$$
\omega(t) \triangleq\left[x^{T}(t), r(t)\right]^{T} \text { and } \theta(t) \triangleq\left[\theta_{1}^{T}(t), \theta_{r}(t)\right]^{T} .
$$

The function $f_{c}(\cdot)$ describes the adaptive law, of which there are many variants. The exact form is not important here and therefore not elaborated upon.

For the discrete-time case the controller structure is

$$
\begin{aligned}
v(t) & =\hat{\theta}_{x}^{T}(t) \varphi_{u}(t)+\hat{\theta}_{u}(t)+\hat{\theta}_{r}(t) r(t) \\
& =\hat{\theta}^{T}(t) \varphi(t) \\
u(t) & =\left\{\begin{array}{ccc}
v(t) & \text { if } & |v(t)| \leq u_{\max } \\
u_{\max } \operatorname{sgn}(v(t)) & \text { if } & |v(t)|>u_{\max }
\end{array}\right. \\
\hat{\theta}(t+1) & =f_{d}(\varphi, \hat{\theta}, t) .
\end{aligned}
$$

Here $\varphi_{y}$ and $\varphi_{u}$ contain a measurement history of the plant output, $y(t)$ and the control input, $u(t)$,respectively, $\hat{\theta} \triangleq\left[\hat{\theta}_{y}^{T}, \hat{\theta}_{u}^{T}, \hat{\theta}_{r}\right]$ and $\varphi \triangleq\left[\varphi_{y}^{T}, \varphi_{u}^{T}, \varphi_{r}\right]$. As in the continuous-time case, the function $f_{d}(\cdot)$ stands for an adaptive law of some form. Here $\varphi$ plays the role a state vector in a nonminimal state-representation of the plant.

For continuous-time systems, the introduction of a limiter seriously affects the adaptive law in a way which is not trivially compensated for. In [11] Monopoli proposed the addition of correcting signals in the adaptive law. The treatment is however, not complete and not quite rigorous in view of the advances in the field since then.

Discrete-time systems on the other hand, do not need any modifications in the adaptive law; standard adaptive laws naturally accommodate saturation effects [3]. 
In both cases of continuous and discrete-time control, a knowledge of the actual control signal amplitude, $u(t)$, i.e. the control signal after saturation is needed. When the measurement is not available, the saturator can be implemented inside the controller, hence effectively moving the saturator inside the controller. A lower bound on the actual, external saturation limit must then be known.

\subsection{Stability of Adaptive Systems with a Saturated Input}

The presence of a limiter ensures bounded inputs. This implies that if the plant is stable, all the state-variables of the plants are bounded. All that remains to be shown is that the signals in the controller remain bounded. In most cases, this boils down to proving the boundedness of the control parameter. For unstable plants, the problem becomes more complicated. It should be noted that the results of Lemmas 2.1 and 2.2 are applicable in this case.

Lemma 1 is applicable to any unstable system with a LTI plant and is therefore valid in that case here. It is also noted that the controller structure is the same as is assumed for Lemma 2, although it is too early to say whether the other conditions of Lemma 2 are satisfied.

\section{Continuous-Time Constrained Adaptive Control}

In continuous-time adaptive control, the general approach is to construct a reference model which describes the desired behavior of the closed-loop system and would be implemented if all the plant parameters were perfectly known. The fundamental assumption made is that a nominal value for the control parameter, $\theta=\theta^{*}$ exists for which the system behaves exactly like the reference model. This is one of the reason behind the restriction that the structure of the plant must known, as it can argued that a knowledge of the structure is necessary to make the plant behave identically to the model by merely choosing the right control parameter. This control parameter is what the adaptive law is designed to search for. It is not guaranteed, although, that it will find the true parameter value. Only, that it will find some value that makes the system behave like the model for the reference inputs used. However, if the system is 
given a sufficiently "rich" set of reference inputs ${ }^{2}$ then the control parameter estimate will converge to the correct value. That the parameter vector is a part of state for the overall system suggests, in view of Lemma 1, that the initial conditions for that parameter vector must also be bounded. This is indeed the case as will be stated in the following lemma. Let $f_{c}(\cdot)$ be such that it is a bounded function of $\theta$ and also bounded for bounded $(\omega, t)$, i.e. $\exists F_{c}(\omega, t)$ such that

$$
\left\|f_{c}(\omega, \theta, \tau)\right\| \leq F_{c}(\omega, t)<\infty \forall \theta
$$

when $t$, and $\omega$ are bounded.

Lemma 2.4 If the controller structure in Eq. (2.11) satisfying Eq. (2.13) is used for system (2.1) then there exists a finite $\theta\left(t_{0}\right)$ such that the origin of the linear subsystem is unstable.

Proof: Instability means that there exists a point, in the linear subsystem, arbitrarily close to the origin and a bounded initial condition for the control parameter that lead to unbounded trajectories.

As in the proofs for Lemmas 2.1 and 2.2, consider the most unstable eigenvalue, $\lambda_{i}$ of the linear subsystem, and the corresponding decoupled, differential equation

$$
\dot{x}_{i}(t)=\lambda_{i} x_{i}(t)+b_{i} u(t)
$$

Without loss of generality assume $b_{i}>0$ and let $x_{i}\left(t_{0}\right)=\varepsilon x_{\max }$, where $0<\varepsilon<1$ and $x_{\max }$ is defined in (2.4). Let $\bar{\omega}$ and $\bar{\theta}$ be the the state vector and the parameter vector, respectively, obtained by excluding the entries corresponding to $x_{i}$ from $\omega$ and $\theta$. The system is linear and in view of Lemma (2.2) only bounded reference inputs are considered, then $\exists \gamma, c_{1}, c_{0}>0$ such that

$$
\|\bar{\omega}(t)\| \leq c_{1} e^{\gamma\left(t-t_{0}\right)}+c_{0} \triangleq \bar{\omega}_{\max }\left(t-t_{0}\right) \forall t \geq t_{0} .
$$

\footnotetext{
${ }^{2}$ The signals that satisfy this richness condition are referred to as persistently exciting in adaptive control theory [1].
} 
Let

$$
F_{c}^{\max }\left(t, t_{0}\right) \triangleq \sup _{\substack{\|\bar{\omega}(t)\| \leq \tilde{\omega}\left(t-t_{0}\right) \\ t_{0} \leq \tau \leq t}} F_{c}(\omega, \tau)
$$

then if $\bar{\theta}\left(t_{0}\right)=0$ it follows that

$$
\|\bar{\theta}(t)\| \leq\left(t-t_{0}\right) F_{c}^{\max }\left(t, t_{0}\right),
$$

and by choosing

$$
\theta_{i}\left(t_{0}\right)=\left(t_{u}-t_{0}\right) F_{c}^{\max }\left(t_{u}, t_{0}\right)\left[1+\frac{\bar{\omega}_{\max }\left(t_{u}-t_{0}\right)}{\varepsilon x_{\max }}\right]
$$

where

$$
t_{u} \triangleq t_{0}+\frac{1}{\operatorname{Re}\left(\lambda_{i}\right)} \log \frac{1}{\varepsilon} .
$$

The result obtained is that

$$
\begin{aligned}
u(t) & =\bar{\theta}^{T}(t) \bar{\omega}(t)+\theta_{i}(t) x_{i}(t) \\
& \geq-\bar{\omega}_{\max }\left(t-t_{0}\right) F_{c}^{\max }\left(t, t_{0}\right)\left(t-t_{0}\right)+\left(\theta_{i}\left(t_{0}\right)-F_{c}^{\max }\left(t, t_{0}\right)\left(t-t_{0}\right)\right) x_{i}(t),
\end{aligned}
$$

giving $u\left(t_{0}\right) \geq 0$, so $\dot{x}_{i}\left(t_{0}\right)$ is increasing and because Eq. (2.14) holds for all $x_{i}(t) \geq$ $\varepsilon x_{\max }$ when $t_{0} \leq t \leq t_{u}$, it follows that

$$
\begin{aligned}
\dot{x}_{i}(t) & =\lambda_{i} x_{i}(t)+b_{i} u(t) \\
& \geq \lambda_{i} x_{i}(t) \quad \forall t \in\left[t_{0}, t_{u}\right]
\end{aligned}
$$

and

$$
x_{i}\left(t_{u}\right) \geq \varepsilon x_{\max } e^{\lambda_{i}\left(t_{u}-t_{0}\right)}=x_{\max }
$$

and from Lemma 2.1, the proof follows.

As before with Lemmas 2.1 and 2.1, Lemma 2.4 applies equally to discrete-time systems. 


\subsection{Conclusion}

The following conclusions can be drawn from Lemmas 2.1, 2.2 and 2.4.

To guarantee stability for an adaptive system as described in Eqns. (2.1), (2.3) and (2.11) where the plant is not necessarily stable the following factors must be included.

1. A bound on the initial conditions of the plant.

2. A bound on the magnitude of the reference input.

3. A bound on the initial conditions of the parameter estimate.

Exactly how these factors contribute to stability will be the main subject of chapters 3 and 4 .

\section{Papers Published on Adaptive Control in the Presence of Constraints}

As stated before, the basic structure for modifying the adaptive law for effects of saturation limits was proposed by Monopoli in 1975 [11]. Papers that have dealt with this problem in the past few years include [23] -[31]. In [23]-[29] it is assumed that the plant is open-loop stable. In $[24,25]$ the problem of discrete time, direct adaptive control is addressed In [27]-[30] the problem of discrete time, adaptive poleplacement control is addressed and by and large all employ projection to guarantee bounded parameter estimates and based on that derive global stability for type-1 plants. In [29] the case of continuous time indirect adaptive control is explored and there again projection is used in the adaptive algorithm and stability results only for open-loop stable plants given. In [31] the proposed approach of Monopoli in [11] is followed in continuous time direct adaptive control, i.e. to modify the adaptive law by augmenting the error signal with correcting signals to establish parameter boundedness as before results apply only to open-loop stable plants. 


\section{Chapter 3}

\section{Adaptive Control of}

\section{Continuous-Time Systems}

In this chapter some new results obtained on the problems described in the previous chapter are stated in Theorems 3.1-3.4 where modified control schemes and stability of the overall system is established. Simulations are presented to support the theoretical derivations.

\section{A First-Order Plant}

Statement of the Problem: A plant with an input-output pair $\left\{u(\cdot), x_{p}(\cdot)\right\}$ is described by the scalar equation

$$
\dot{x}_{p}(t)=a_{p} x_{p}(t)+b_{p} u(t)
$$

where $a_{p}$ and $b_{p}$ are unknown, but the sign of $b_{p}$ is assumed to be known. The input $u(t)$ is additionally subject to the magnitude constraint

$$
|u(t)| \leq u_{0}
$$

where $u_{0}$ is a known constant. 
A reference model is described by the first-order differential equation

$$
\dot{x}_{m}(t)=a_{m} x_{m}(t)+b_{m} r(t)
$$

where $a_{m}$ is a known negative constant, $b_{m}$ is known, and $r(t)$ is a piecewise-continuous bounded function whose magnitude is such that

$$
|r(t)| \leq r_{0} .
$$

It is assumed that $a_{m}, b_{m}$ and $r(t)$ have been chosen so that $x_{m}(t)$ represents the output desired of the plant at time $t$. The aim is to determine a bounded control input $u(t)$ so that all signals in the system remain bounded, for a given set of initial conditions, and $x_{p}$ tracks $x_{m}$ as closely as possible.

If the plant parameter is known, a feedback controller of the form

$$
u(t)=\theta^{*} x_{p}(t)+k^{*} r(t), \quad \text { where } \theta^{*}=\frac{a_{m}-a_{p}}{b_{p}} \quad \text { and } k^{*}=\frac{b_{m}}{b_{p}}
$$

suffices. Since $a_{p}, b_{p}$ and hence $\theta^{*}, k^{*}$ are unknown the control input is chosen to have the form

$$
\begin{aligned}
& v(t)=\begin{array}{ccc}
\theta(t) x_{p}(t)+k(t) r(t) \\
u(t) & \text { if } & |v(t)| \leq u_{0}
\end{array} \\
& u(t)=\left\{\begin{array}{ccc}
v(t) \mid>u_{0} \\
u_{0} \operatorname{sgn}(v(t)) & \text { if } & \mid v(t)
\end{array}\right.
\end{aligned}
$$

where $\theta(t)$ is a time-varying control parameter. Then the closed-loop system can be described by

$$
\dot{x}_{p}(t)=\left(a_{p}+b_{p} \theta(t)\right) x_{p}(t)+b_{p} \Delta u(t)+b_{p} k(t) r(t)
$$

where $\Delta u(t)=u(t)-v(t)$. If the output error $e(t)$ and the parameter errors $\phi(t)$ and $\psi(t)$ are defined as

$$
e(t) \triangleq x_{p}(t)-x_{m}(t), \quad \phi(t) \triangleq \theta(t)-\theta^{*}(t), \quad \psi(t)=k(t)-k^{*}
$$


the obtained error equation is from Eqs. (3.2) and (3.4) as

$$
\dot{e}(t)=a_{m} e(t)+b_{p} \phi(t) x_{p}(t)+b_{p} \psi(t) r(t)+b_{p} \Delta u(t) .
$$

In order to remove the effect of $\Delta u$, which can be considered as a known disturbance, a signal $e_{\Delta}(t)$ is generated as the output of a differential equation

$$
\dot{e}_{\Delta}(t)=a_{m} e_{\Delta}(t)+k_{\Delta}(t) \Delta u(t) \quad e_{\Delta}\left(t_{0}\right)=0 .
$$

If $e_{u}(t)=e(t)-e_{\Delta}(t)$, it is obtained that

$$
\dot{e}_{u}(t)=a_{m} e_{u}(t)+b_{p} \phi(t) x_{p}(t)+b_{p} \psi r(t)+\kappa(t) \Delta u(t) .
$$

Where $\kappa(t)=b_{p}-k_{\Delta}(t)$. Eq. (3.5) is in a standard error model form for which the following adaptive laws can be used

$$
\begin{aligned}
\dot{\phi}(t) & =-\gamma_{1} \operatorname{sgn}\left(b_{p}\right) e_{u} x_{p} \\
\dot{\psi}(t) & =-\gamma_{2} \operatorname{sgn}\left(b_{p}\right) e_{u} r \\
\dot{\kappa}(t) & =-\gamma_{3} e_{u} \Delta u
\end{aligned}
$$

where $\gamma_{i}>0 i=1,2,3$. This results in a Lyapunov function

$$
V=\frac{1}{2}\left[e_{u}^{2}+\left|b_{p}\right|\left(\frac{1}{\gamma_{1}} \phi^{2}+\frac{1}{\gamma_{2}} \psi^{2}\right)+\frac{1}{\gamma_{3}} \kappa^{2}\right]
$$

since $\dot{V}=a_{m} e_{u}^{2} \leq 0$. Hence $\phi(t), \psi(t), \kappa(t)$ and $e_{u}(t)$ are bounded $\forall t \geq t_{0}$. From (3.7) it can be deduced that $\exists \phi_{\max }<\infty$ such that $|\phi(t)|<\phi_{\max }$ and $|\psi(t)|<\alpha \phi_{\max } \forall t \geq$ $t_{0}$, where

$$
\alpha=\sqrt{\frac{\gamma_{1}}{\gamma_{2}}}
$$

Theorem 3.1 The adaptive system in Eqs. (3.1), (3.2), (3.3), and (3.6) has bounded 
solutions $\forall|r(t)| \leq r_{0}$ if
(i) $\left|x_{p}\left(t_{0}\right)\right| \leq\left|\frac{b_{p} u_{0}}{a_{p}}\right|$ and
(ii) $\sqrt{V\left(t_{0}\right)} \leq \sqrt{\frac{\left|b_{p}\right|}{2 \gamma_{1}}} \frac{\left|a_{m}\right|-k^{*} \frac{r_{0}}{u_{0}}\left|a_{p}\right|}{\left|b_{p}\right|+\alpha \frac{r_{0}}{u_{0}}\left|a_{p}\right|}$.

Further,

$$
\left|x_{p}(t)\right| \leq\left|\frac{b_{p} u_{0}}{a_{p}}\right| \quad \forall t \geq t_{0} .
$$

Proof: See Appendix A

Theorem 1 implies that adaptive control of a first-order plant in Eq. (3.1) leads to bounded solutions with the controller in Eq. (3.3) and the adaptive law in Eq. (3.6) provided conditions (i) and (ii) are met. The boundedness of all signals in the adaptive system was established in two steps. First the parameter error $\phi(\cdot)$ was shown to be bounded which was done using the adaptive law in (3.6). Next the boundedness of the state $x_{p}$ of the plant was derived, if the initial conditions are restricted to remain within a bounded set. Additional comments regarding the bounds on the initial conditions are made at the end of section 2 ..

\section{State Variables Accessible}

In this section the plant considered is an $n$th order one, whose states are accessible and described by the vector differential equation

$$
\dot{x}_{p}(t)=A_{p} x_{p}(t)+b_{p} u(t)
$$

where the entries $A_{p} \in \mathbb{R}^{n \times n}$ and $b_{p} \in \mathbb{R}^{n}$ are unknown. It is assumed that a reference model described by

$$
\dot{x}_{m}(t)=A_{m} x_{m}(t)+b_{m} r(t)
$$

can be determined where $r(t)$ is a piecewise-continuous bounded function with $|r(t)| \leq$ $r_{0}$, and $A_{m} \in \mathbb{R}^{n \times n}$ is an asymptotically stable matrix, which is such that a vector 
$\theta^{*}$ and a scalar $k^{*}$ exists and are solutions of

$$
\begin{aligned}
A_{p}+b_{m} \theta^{* T} & =A_{m} \\
k^{*} b_{p} & =b_{m} .
\end{aligned}
$$

As in the scalar case the, input $u(t)$ is subjected to the magnitude constraint

$$
|u(t)| \leq u_{0}
$$

where $u_{0}$ is a known constant. The aim is to determine a bounded control input $u(t)$ so that $x_{p}$ tracks $x_{m}$ as closely as possible while all signals in the system remain bounded. The main result is summarized in the following theorem.

Theorem 3.2 Let an adaptive controller be chosen as

$$
\begin{aligned}
& v(t)=k(t) \theta^{T}(t) x_{p}(t)+k(t) r(t) \\
& u(t)=\left\{\begin{array}{ccc}
v(t) & \text { if } & |v(t)| \leq u_{0} \\
u_{0} \operatorname{sgn}(v(t)) & \text { if } & |v(t)|>u_{0}
\end{array}\right. \\
& \dot{\phi}(t)=-\Gamma x_{p} e_{u}^{T} P b_{m} \\
& \dot{\psi}(t)=-\gamma_{1} u e_{u}^{T} P b_{m} \\
& \dot{e}_{\Delta}(t)=A_{m} e_{\Delta}(t)+b_{m} k(t)^{-1} \Delta u(t) \quad e_{\Delta}\left(t_{0}\right)=0 \\
& e_{u}(t) \triangleq e(t)-e_{\Delta}(t) \quad \phi(t) \triangleq \theta(t)-\theta^{*}(t) \quad \psi(t) \triangleq \frac{1}{k^{*}}-\frac{1}{k(t)}
\end{aligned}
$$

where $A_{m}^{T} P+P A_{m}=-Q$ and $Q=Q^{T}>0$. Let $q_{0}=\lambda_{\min }(Q), \alpha=\sqrt{\frac{\gamma_{1}}{\lambda_{\max }(\Gamma)}}, p_{b}=$ $\left\|P b_{m}\right\|$ and $\rho=\sqrt{\frac{\lambda_{\max }(P)}{\lambda_{\min }(P)}}$, where $\lambda_{\max }$ and $\lambda_{\min }$ denote the maximum and minimum eigenvalue, respectively. If a Lyapunov function candidate $V$ is defined as

$$
V\left(e_{u}, \phi, \psi\right)=e_{u}^{T} P e_{u}+\phi^{T} \Gamma^{-1} \phi+\frac{1}{\gamma_{1}} \psi^{2} \quad \Gamma=\Gamma^{T}>0 \quad \gamma_{1}>0
$$


then the adaptive system in Eq. (3.10) has bounded solutions if

$$
\begin{aligned}
\text { (i) } x_{p}^{T}\left(t_{0}\right) P x_{p}\left(t_{0}\right) & <\lambda_{\min }(P)\left[\frac{2 p_{b} u_{0}}{\left|k^{*}\right|\left|2 p_{b}\right|\left|\theta^{*}\right|\left|-q_{0}\right|}\right]^{2} \\
\text { and (ii) } \sqrt{V\left(t_{0}\right)} & <\frac{1}{\sqrt{\lambda_{\max }(\Gamma)}} \frac{q_{0}-\rho\left|k^{*}\right| \frac{r_{0}}{u_{0}}\left|2 p_{b}\left\|\theta^{*}\right\|-q_{0}\right|}{2 p_{b}+\alpha\left|k^{*}\right|\left[q_{0}+2 p_{b}\left\|\theta^{*}\right\|\right]} .
\end{aligned}
$$

Further,

$$
x_{p}^{T}(t) P x_{p}(t) \leq \lambda_{\min }(P) \frac{2 p_{b} u_{0}}{\left|k^{*}\right|\left|2 p_{b}\right|\left|\theta^{*} \|-q_{0}\right|} \quad \forall t \geq t_{0} .
$$

Proof: See Appendix A

\section{Remarks:}

1. Theorems 3.1 and 3.2 imply that if the initial conditions of the state as well as the parameter error lie within a certain bound, then the adaptive system will have bounded solutions. The local nature of the stability result is to be expected in view of Lemmas 2.1-2.4, since the control input was restricted to lie within certain limits. This condition can only be removed by restricting the open-loop poles to lie in the left half plane. Theorems 3.1-3.4 extend this statement to the adaptive case, where the control parameter is also a state-variable of the overall nonlinear system, and hence requires not only a bound on $\left\|x\left(t_{0}\right)\right\|$ but also on the norm of the parameter error $\left\|\phi\left(t_{0}\right)\right\|$.

2. Condition (ii) implies that prior information regarding an upper bound on $\left\|\theta^{*}\right\|$ needs to be known so that the matrix $Q$ and hence $q_{0}$ can be chosen to make the right hand side in condition (ii) positive. Since the feedback control input has a component due to the external input and a component due to the plant-model mismatch, it is not surprising that for a given parameter $\left\|\theta^{*}\right\|$, the scaling factor $r_{0} / u_{0}$ must be small. That is, the magnitude of the reference input $r(t)$ and, hence, of the state of the model $x_{m}$ that the plant state must track, must be appropriately scaled. As the mismatch between the plant and the model gets reduced, the factor $r_{0} / u_{0}$ can be increased. 
3. It should be noted that the adaptive algorithm in Eq. (3.12) leads to the boundedness of the control parameter $\theta$. Since the plant input $u$ is always bounded, this implies that if the plant is open-loop stable, the boundedness of the remaining signals in the closed-loop system immediately follows, without any restriction on the initial conditions of the plant or the controller, leading to global stability. Conditions (i) and (ii) in Theorems 3.1 and 3.2 are therefore required only for unstable plants.

4. It is interesting to note that the results will hold for any adaptive law which guarantees boundedness of the parameter error and will therefore extend to the algorithms proposed in [31] as well.

\section{Output Feedback}

Now a look will be taken at the case when only the output is available for feedback. The plant considered is single-input single-output system described by

$$
\dot{x}_{p}(t)=A_{p} x_{p}(t)+b_{p} u(t) \quad y_{p}(t)=h_{p}^{T} x_{p}(t)
$$

where the input $u$ is subjected to the constraint

$$
|u(t)| \leq u_{0} \quad \forall t \geq t_{0},
$$

and $x_{p}: \mathbb{R}^{+} \rightarrow \mathbb{R}^{n}$ is the $n$-dimensional state vector of the plant. Eq. (3.15) is written equivalently as a transfer function

$$
W_{p}(s)=k_{p} \frac{Z_{p}(s)}{R_{p}(s)}=h_{p}^{T}\left(s I-A_{p}\right)^{-1} b_{p}
$$

where $Z_{p}(s)$ and $R_{p}(s)$ are monic polynomials in the differential operator $s$.

The following standard assumptions are made regarding $W_{p}(s)$ :

1. The sign of the high frequency gain, $k_{p}$, is known. 
2. An upper bound, $(n)$, on the order of the plant is known.

3. The relative degree, $\left(n^{*}\right)$, of the plant is known.

4. The roots of $Z_{p}(s)$ are known to be in the open left half of the complex plane, i.e. $Z_{p}(s)$ is Hurwitz.

5. The system $\left\{h_{p}^{T}, A_{p}, b_{p}\right\}$ is controllable and observable.

The objective is to let the plant output $y_{p}(t)$ follow a reference trajectory $y_{m}(t)$ as closely as possible. To achieve that a a reference model is introduced, described by a linear time-invariant, asymptotically stable system with an input-output pair $\left\{r(\cdot), y_{m}(\cdot)\right\}$, and described by a transfer function

$$
W_{m}(s)=k_{m} \frac{Z_{m}(s)}{R_{m}(s)}
$$

with a relative degree $n_{m}^{*}>n^{*}$, where $Z_{m}(s)$ and $R_{m}(s)$ are monic polynomials in the differential operator $s$, and $k_{m}$ is assumed to be positive. The reference input $r(t)$ is assumed to be a uniformly bounded piecewise-continuous function of time, and that

$$
|r(t)| \leq r_{0} \quad \forall t \geq t_{0}
$$

Similar to the standard adaptive controller in [1], a controller is chosen of the form

$$
\begin{aligned}
\dot{\omega}_{1}(t) & =\Lambda \omega_{1}(t)+l u(t) \\
\dot{\omega}_{2}(t) & =\Lambda \omega_{2}(t)+l y_{p}(t) \\
\omega(t) & =\left[r(t), \omega_{1}^{T}(t), y_{p}(t), \omega_{2}^{T}(t)\right] \\
\theta(t) & =\left[k(t), \theta_{1}^{T}(t), \theta_{0}(t), \theta_{2}^{T}(t)\right] \\
v(t) & =\theta(t)^{T} \omega(t) \\
u & =\left\{\begin{array}{lll}
v(t) & \text { if }|v(t)| \leq u_{0} \\
u_{0} \operatorname{sgn}(v(t)) & \text { if }|v(t)|>u_{0}
\end{array}\right.
\end{aligned}
$$


where $k: \mathbb{R}^{+} \rightarrow, \theta_{1}, \omega_{1}: \mathbb{R}^{+} \rightarrow \mathbb{R}^{n-1}, \theta_{0}: \mathbb{R}^{+} \rightarrow \mathbb{R}, \theta_{2}, \omega_{2}: \mathbb{R}^{+} \rightarrow \mathbb{R}^{n-1}, \Lambda$ is an asymptotically stable matrix and $\operatorname{det}(s I-\Lambda)=\lambda(s)$. It follows that when the control parameters $k(t), \theta_{1}(t), \theta_{0}(t), \theta_{2}(t)$ assume the constant values $k_{c}, \theta_{1 c}, \theta_{0 c}, \theta_{2 c}$, respectively, the transfer function of the feedforward and the feedback controllers become respectively

$$
\frac{\lambda(s)}{\lambda(s)-C(s)} \quad \text { and } \quad \frac{D(s)}{\lambda(s)}
$$

where $C(s)$ is determined by $\theta_{1 c}$ and $D(s)$ by $\theta_{2 c}, \theta_{0 c}$ or

$$
\frac{C(s)}{\lambda(s)}=\theta_{1 c}^{T}(s I-\Lambda)^{-1} l, \quad \frac{D(s)}{\lambda(s)}=\theta_{0 c}+\theta_{2 c}^{T}(s I-\Lambda)^{-1} l .
$$

The overall transfer function of the plant together with the controller can be expressed as

$$
W_{o}(s)=\frac{k_{c} k_{p} Z_{p}(s) \lambda(s)}{(\lambda(s)-C(s)) R_{p}(s)-k_{p} Z_{p}(s) D(s)}
$$

where $k_{c}=\frac{k_{m}}{k_{p}}$. If we now chose $\lambda(s)$ so that it contains $Z_{m}(s)$ as a factor, $\lambda(s)=$ $Z_{m}(s) \lambda_{1}(s)$ then (3.18) becomes

$$
W_{o}(s)=\frac{k_{c} k_{p} Z_{p}(s) \lambda_{1}(s) Z_{m}(s)}{(\lambda(s)-C(s)) R_{p}(s)-k_{p} Z_{p}(s) D(s)} .
$$

The existence of the control parameter $\theta^{*}$, which lets $W_{o}(s) \equiv W_{m}(s)$, is equivalent to the existence of the polynomials $C^{*}(s), D^{*}(s)$, and the gain $k^{*}$ such that the denominator polynomial in Eq. (3.19) becomes $Z_{p}(s) \lambda_{1}(s) R_{m}(s)$. Identifying $R_{p}(s)$ with $Q(s),-k_{p} Z_{p}(s)$ with $P(s)$ and $Z_{p}(s) \lambda_{1}(s) R_{m}(s)$ with $Q^{*}(s)$ the problem is to determine $C^{*}(s)$ and $D^{*}(s)$ such that

$$
\left[\lambda(s)-C^{*}(s)\right] Q(s)+D^{*}(s) P(s)=Q^{*}(s),
$$

which is the Bezout's identity and it guarantees the existence of $D^{*}(s)$, and $C^{*}(s)$ if $Q(s)$ and $P(s)$ (or equivalently, $R_{p}(s)$ and $Z_{p}(s)$ ) are relatively prime, i.e. the controllability/observability assumption made earlier are sufficient conditions for a 
solution to exist. The differential equation describing the plant together with the controller can be represented as

$$
\begin{aligned}
& \dot{x}_{p}(t)=A_{p} x_{p}(t)+b_{p}\left(\theta(t)^{T} \omega(t)+\Delta u(t)\right) \\
& \dot{\omega}_{1}(t)=\Lambda \omega_{1}(t)+l\left(\theta(t)^{T} \omega(t)+\Delta u(t)\right) \\
& \dot{\omega}_{2}(t)=\Lambda \omega_{2}(t)+l\left(h_{p}^{T} x_{p}\right)
\end{aligned}
$$

where $\Delta u(t)=u(t)-v(t)$. With the controller in Eq. (3.16), and the parameter errors defined as

$$
\begin{aligned}
& \psi(t)=k(t)-k^{*} \quad \phi_{0}(t)=\theta_{0}(t)-\theta_{0}^{*} \quad \phi_{1}(t)=\theta_{1}(t)-\theta^{*} \\
& \phi_{2}(t)=\theta_{2}(t)-\theta_{2}^{*}, \quad \phi(t)=\left[\psi(t), \phi_{1}^{T}(t), \phi_{0}(t), \phi_{2}^{T}(t)\right] .
\end{aligned}
$$

the overall system becomes

$$
\dot{x}(t)=A_{m n} x(t)+b_{m n}\left[\phi^{T}(t) \omega(t)+k^{*} r(t)+\Delta u(t)\right]
$$

where $\Delta u(t) \triangleq u(t)-v(t)$

$$
\begin{aligned}
& A_{m n}=\left[\begin{array}{ccc}
A_{p}+b_{p} \theta_{0}^{*} h_{p}^{T} & b_{p} \theta_{1}^{* T} & b_{p} \theta_{2}^{* T} \\
l \theta_{0}^{*} h_{p}^{T} & \Lambda+l \theta_{1}^{* T} & l \theta_{2}^{* T} \\
l h_{p}^{T} & 0 & \Lambda
\end{array}\right] \quad b_{m n}=\left[\begin{array}{c}
b_{p} \\
l \\
0
\end{array}\right] \\
& h_{m n}=\left[h_{p}^{T}, 0,0\right]^{T}, \quad \text { and } \quad x=\left[x_{p}^{T}, \omega_{1}, \omega_{2}\right] .
\end{aligned}
$$

Since $W_{o}(s) \equiv W_{m}(s)$ when $\theta(t) \equiv \theta^{*}$ it follows that the reference model can be described non-minimally by the $(3 n-1)^{\text {th }}$ order differential equation

$$
\dot{x}_{m n}(t)=A_{m n} x_{m n}(t)+b_{m n} k^{*} r(t) \quad y_{m}(t)=h_{m n}^{T} x_{m n}(t)
$$

where $x_{m n}(t)=\left[x^{*}(t), \omega_{1}^{*}(t), \omega_{2}^{*}(t)\right]$. The signals in $x_{m n}(t)$ can be considered as 
signals in the reference model corresponding to $x(t)$ in the overall system. Hence, the error equation for the overall system may be expressed as

$$
\dot{e}(t)=A_{m n} e(t)+b_{m n}\left[\phi^{T}(t) \omega(t)+\Delta u(t)\right], \quad e_{1}(t)=h_{m n}^{T} e(t) .
$$

If $e_{1}=y_{p}-y_{m}$, the error model is given by

$$
e_{1}(t)=\frac{k_{p}}{k_{m}} W_{m}(s)\left[\phi^{T}(t) \omega(t)\right]
$$

in the absence of the limiter. With the limiter, if $\Delta u=u-v$, it can be shown in a straightforward manner that

$$
e_{1}(t)=\frac{k_{p}}{k_{m}} W_{m}(s)\left[\phi^{T}(t) \omega(t)+\Delta u(t)\right] .
$$

In what follows, the cases $n^{*}=1$ and $n^{*} \geq 2$ are treated separately to describe the adaptive law which establishes the boundedness of the parameter error. Once this is done, the boundedness of the rest of the state variables of the adaptive system for arbitrary $n^{*}$ is established in section 2.3.3.

\subsection{Adaptive law $n^{*}=1$}

As in the standard adaptive control problem, the adaptive law is considerably simpler when $n^{*}=1$ than when $n^{*} \geq 2$ since in the former case $W_{m}(s)$ can be made strictly positive real (SPR). The $\Delta u(t)$ term in Eq. (3.23) is compensated for by generating the signal

$$
e_{\Delta}(t)=W_{m}(s) k_{\Delta}(t) \Delta u(t)
$$

where $k_{\Delta}(t)$ is a time-varying gain, adjusted according to an adaptive law that is to be determined. If a compensated error $e_{u 1}$ is defined as $e_{u 1}=e_{1}-e_{\Delta}$, and a parameter error $\psi_{\Delta}$ as $\psi_{\Delta}=1-k^{*} k_{\Delta}$, it is obtained that

$$
e_{u 1}(t)=\frac{1}{k^{*}} W_{m}(s)\left[\phi_{u}^{T}(t) \omega_{u}((t)]\right.
$$


where

$$
\phi_{u}(t)=\left[\psi_{\Delta}, \phi^{T}(t)\right]^{T} \quad \omega_{u}(t)=\left[\Delta u(t), \omega^{T}(t)\right]^{T} .
$$

The error model is now have in a recognizable form, and hence an adaptive law can be found by inspection as

$$
\dot{\phi}=-\operatorname{sgn}\left(k_{p}\right) \Gamma \omega e_{u 1} \quad \dot{\psi}_{\Delta}=-k^{*} \gamma_{\Delta} e_{u 1} \Delta u \quad \text { where } \Gamma=\Gamma^{T}>0 \text { and } \gamma_{\Delta}>0 .
$$

It should be noted that the adaptive law in Eq. (3.24) is implementable since $\dot{k}_{\Delta}=$ $\gamma_{\Delta} e_{u 1} \Delta u$. The use of Kalman-Yakubovich lemma then gives us that $e_{u 1}, \phi, \psi_{\Delta} \in \mathcal{L}^{\infty}$, with a Lyapunov function

$$
V\left(e_{u}, \phi, \psi_{\Delta}\right)=e_{u}^{T} P e_{u}+\frac{1}{\left|k^{*}\right|} \phi^{T} \Gamma^{-1} \phi+\frac{1}{\gamma_{\Delta} k^{* 2}} \psi_{\Delta}^{2}
$$

where $e_{u}$ is the state corresponding to the output $e_{u 1}$.

\subsection{Adaptive Law, $n^{*} \geq 2$}

Here, as in the standard adaptive control problem, an augmented error is used to determine the adaptive laws. First define the signal

$$
e_{\Delta}(t)=k_{\Delta}(t) W_{m}(s) \Delta u(t)
$$

where $k_{\Delta}(t)$ is a time-varying gain, to compensate for the term $\Delta u(t)$ in Eq. (3.23). If $e_{u 1}=e_{1}-e_{\Delta}$, then

$$
e_{u 1}(t)=\frac{1}{k^{*}} W_{m}(s)\left[\phi^{T}(t) \omega(t)\right]+\psi_{\Delta}(t) W_{m}(s) \Delta u(t)
$$

where $\psi_{\Delta}(t)=\frac{1}{k^{*}}-k_{\Delta}(t)$. Then the auxiliary error and the augmented error are determined as

$$
\begin{gathered}
e_{2}(t)=\left[\theta^{T} W_{m}(s) I-W_{m}(s) \theta^{T}(t)\right] \omega(t) \\
\varepsilon_{1 u}(t)=e_{u 1}(t)+k_{1}(t) e_{2}(t) .
\end{gathered}
$$


It follows that

$$
\varepsilon_{1 u}=\frac{1}{k^{*}} \phi^{T}(t) \xi(t)+\psi_{\Delta}(t) \xi_{\Delta}(t)+\psi_{1}(t) e_{2}(t)+\delta(t)
$$

where $\xi(t)=W_{m}(s) I \omega(t), \xi_{\Delta}(t)=W_{m}(s) \Delta u(t)$, and $\delta(t)$ is an exponentially decaying term due to initial conditions. The adaptive laws can be found by inspection as

$$
\begin{aligned}
& \dot{\theta}(t)=\dot{\phi}(t)=-\operatorname{sgn}\left(k_{p}\right) \Gamma \frac{\varepsilon_{1 u}(t) \xi(t)}{1+\xi^{T}(t) \xi(t)} \\
& \dot{k}_{1}(t)=\dot{\psi}_{1}(t)=-\gamma_{1} \frac{\varepsilon_{1 u}(t) e_{2}(t)}{1+\xi^{T}(t) \xi(t)} \\
& \dot{k}_{\Delta}(t)=-\dot{\psi}_{\Delta}(t)=\gamma_{\Delta} \frac{\varepsilon_{1 u}(t) \xi_{\Delta}(t)}{1+\xi^{T}(t) \xi(t)}
\end{aligned}
$$

which gives us that $\varepsilon_{1 u}, \phi, \psi_{1}, \psi_{\Delta} \in \mathcal{L}^{\infty}$ with

$$
V\left(\phi, \psi_{1}, \psi_{\Delta}\right)=\frac{1}{2}\left[\frac{1}{\left|k^{*}\right|} \phi^{T} \Gamma^{-1} \phi+\frac{1}{\gamma_{1}} \psi_{1}^{2}+\frac{1}{\gamma_{\Delta}} \psi_{\Delta}^{2}\right]
$$

as a Lyapunov function.

\subsection{Boundedness of Signals}

The results in sections 2.3.1 and 2.3.2 imply that adaptive laws can be found which imply that, for any relative degree,

$$
\|\phi(t)\| \leq \phi_{\max } \quad \forall t \geq t_{0}
$$

in Eq. (3.21). Define $P$ as the symmetric positive definite solution to Lyapunov equation, and $\rho$ as the ratio between the maximum and the minimum eigenvalues of $P$ so that

$$
A_{m n}^{T} P+P A_{m n}=-Q \quad \rho=\sqrt{\frac{\lambda_{\max }(P)}{\lambda_{\min }(P)}}
$$


Let $q_{0}=\lambda_{\min }(Q)$ and $p_{b}=\left\|b_{m n}^{T} P\right\|, \bar{\omega}=\left[\omega_{1}^{T}, y_{p}, \omega_{2}^{T}\right]^{T}, \bar{\phi}=\left[\phi_{1}^{T}, \phi_{0}, \phi_{2}^{T}\right]^{T}$ and $\overline{\theta^{*}}=\left[\theta_{1}^{T}, \theta_{0}, \theta_{2}^{T}\right]^{T}$. If

$$
C=\left[\begin{array}{ccc}
0 & I & 0 \\
h_{p}^{T} & 0 & 0 \\
0 & 0 & I
\end{array}\right]
$$

then $\bar{\omega}=C x$. Also, since $\phi=\left[k, \bar{\phi}^{T}\right]^{T}$, we have $\|\bar{\phi}(t)\| \leq \phi_{\max }$ and $|k(t)| \leq\left|k^{*}\right|+\phi_{\max }$ for all $t \geq t_{0}$. Now the main result will be stated, which proves the boundedness of all signals in the adaptive system.

Theorem 3.3 The system in Eq. (3.21) has bounded solutions if

$$
\begin{aligned}
& \text { (i) } \sqrt{V\left(t_{0}\right)} \leq \frac{1}{\sqrt{2\left|k^{*}\right| \lambda_{\max }(\Gamma)}} \frac{q_{0}-\left|k^{*}\right| \rho \frac{r_{0}}{u_{0}}\left|2 p_{b}\right|\left|\bar{\theta}^{* T} C \|-q_{0}\right|}{\rho \frac{r_{0}}{u_{0}}\left|2 p_{b}\right|\left|\bar{\theta}^{* T} C\left\|-q_{0} \mid+2 p_{b}\right\| C \|\right.} \\
& \text { (ii) } \quad x^{T}\left(t_{0}\right) P x\left(t_{0}\right) \leq \lambda_{\min }(P)\left[\frac{2 p_{b} u_{0}}{\left|2 p _ { b } \left\|\left|\bar{\theta}^{* T} C \|-q_{0}\right|\right.\right.}\right]^{2} .
\end{aligned}
$$

Further

$$
x^{T}(t) P x(t) \leq \lambda_{\min }(P)\left[\frac{2 p_{b} u_{0}}{\left|2 p _ { b } \left\|\left|\bar{\theta}^{* T} C \|-q_{0}\right|\right.\right.}\right]^{2} \quad \forall t \geq t_{0} .
$$

Proof: See Appendix A.

It is noted that condition (ii) in Theorem 3.3 is quite difficult to check since the quantity on the right hand side requires a fair amount of knowledge of the plant parameters. It may be argued that the bounds on the initial conditions in (i) and (ii) establish the existence of such values for which the overall adaptive system is stable. However as is seen in the simulation results in the next section, these bounds are indeed conservative and the initial conditions can be increased further before instability occurs. 


\section{Robustness}

The stability results given in Theorems 3.1-3.3 can readily be extended to include bounded disturbances. Here only the most general case of Theorem 3.3 will be treated as the modifications needed for 3.1 and 3.2 follow quite trivially.

Assume that a disturbance $\nu(t)$, where $|\nu(t)| \leq \nu_{0}$, enters the system, between the limiter and the plant (see Fig 3-1). The problem is to adaptively control such a plant in the presence of parametric uncertainties, and input constraints, so that all the signals in the closed-loop system remain bounded and the output $y_{p}$ tracks the model output $y_{m}$ as closely as possible.

\subsection{Disturbances}

Disturbances due to many different sources can be accounted for with a model in Fig. 3-1, and are not limited to input disturbances. Unmodelled dynamics, as well as measurement disturbances can relatively easily be cast into the form shown in Fig. 3-1. It is fairly easy to see that the latter can be reduced to the problem in Fig 3-1 while the former may not be obvious. That case will therefore be investigated further.

\section{Unmodelled Dynamics}

Let $\widetilde{W}_{p}(s)$ stand for a additive perturbation of the plant so that

$$
\bar{W}_{p}(s)=W_{p}(s)+\widetilde{W}_{p}(s)
$$

then it can be shown [1] that this perturbation can be considered as a input- disturbance of the form in Fig. 3-1, where

$$
\nu(t)=\left[1-W_{1}(s) W_{m}(s)\right] W_{m}^{-1}(s) \widetilde{W}_{p}(s) u_{c}(t)
$$




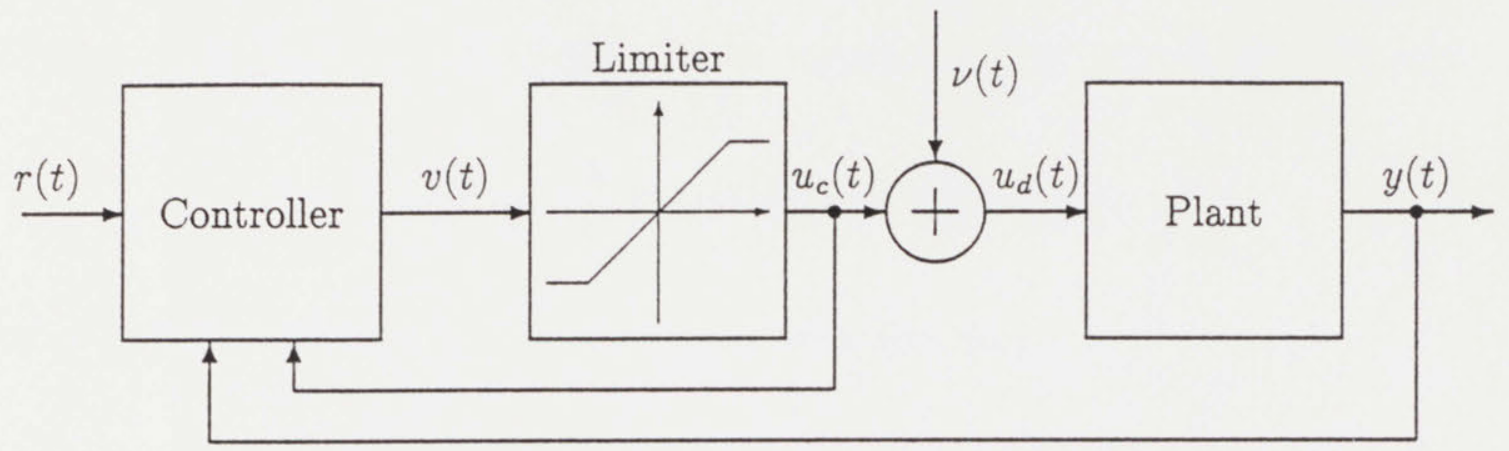

Figure 3-1: System Block-Diagram with Input-Disturbances.

The system with added input-disturbances. The figure defines additional variables $u_{c}(t)$ as controller output, $\nu(t)$ as the external disturbance, and $u_{d}(t)$ as the actual input with the disturbance included.

where $W_{1}(s)$ is defined as the feedback controller transfer function which from Eq. (3.17) is equal to

$$
W_{1}(s) \triangleq \frac{D^{*}(s)}{\lambda(s)}=\theta_{0}^{*}+\theta_{2}^{* T}(s I-\Lambda)^{-1} l .
$$

If $\widetilde{W}_{p}(s)$ is stable and has a relative degree higher than or equal to that of $W_{m}(s)$ then $\nu(t)$ will be bounded as $u_{c}(t)$ is bounded. It is noted that the introduction of the limiter simplifies the problem as it makes it possible to deduce the boundedness of $\nu$, whereas without the limiter that cannot be done.

\subsection{Modification of Adaptive Law}

As the disturbance enters additively at the input then the actual input that enters the plant is

$$
u_{d}(t)=u_{c}(t)+\nu(t)
$$


The system as described in Eq (3.21) without disturbance, becomes with disturbances

$$
\dot{x}(t)=A_{m n} x(t)+b_{m n}\left[\phi^{T}(t) \omega(t)+k^{*} r(t)+\Delta u(t)+\nu(t)\right] .
$$

After the correcting signal generated from $\Delta u(t)=u_{c}(t)-v(t)$ has been subtracted from the error equation, the corrected error equation for the $n^{*}=1$ case becomes

$$
e_{u 1}(t)=\frac{1}{k^{*}} W_{m}(s)\left[\phi_{u}^{T}(t) \omega_{u}((t)+\nu(t)]\right.
$$

For $n^{*} \geq 2$ the equation for the augmented error becomes

$$
\varepsilon_{1 u}=\frac{1}{k^{*}} \phi^{T}(t) \xi(t)+\psi_{\Delta}(t) \xi_{\Delta}(t)+\psi_{1}(t) e_{2}(t)+\frac{1}{k^{*}} W_{m}(s) \nu(t)+\delta(t) .
$$

Because $W_{m}(s)$ is asymptotically stable the disturbance term can be replaced by $\bar{\nu}(t)$ which is also bounded by

$$
|\bar{\nu}(t)| \leq\left\|\frac{1}{k^{*}} W_{m}(s)\right\|_{\infty} \nu_{0} \triangleq \bar{\nu}_{0}
$$

Several methods have been developed to guarantee parameter boundedness. The Dead-Zone scheme is the most basic one, but others include. Bound on $\|\theta\|$, the $\sigma$ modification scheme, and the $e_{1}$-modification scheme [1]. Using the dead-zone method 
as an example for the $n^{*} \geq 2$ case, the modified adaptive laws become

$$
\begin{aligned}
& \dot{\phi}(t)=\left\{\begin{array}{ccc}
-\operatorname{sgn}\left(k_{p}\right) \Gamma \frac{\varepsilon_{1 u}(t) \xi(t)}{1+\xi^{T}(t) \xi(t)} & \text { if } & \left|\varepsilon_{u 1}\right| \geq \bar{\nu}_{0}+\delta \\
0 & \text { if } & \left|\varepsilon_{u 1}\right|<\bar{\nu}_{0}+\delta
\end{array}\right. \\
& \dot{\psi}_{1}(t)=\left\{\begin{array}{ccc}
-\gamma_{1} \frac{\varepsilon_{1 u}(t) e_{2}(t)}{1+\xi^{T}(t) \xi(t)} & \text { if } & \left|\varepsilon_{u 1}\right| \geq \bar{\nu}_{0}+\delta \\
0 & \text { if } & \left|\varepsilon_{u 1}\right|<\bar{\nu}_{0}+\delta
\end{array}\right. \\
& \dot{\psi}_{\Delta}(t)=\left\{\begin{array}{ccc}
-\gamma_{\Delta} \frac{\varepsilon_{1 u}(t) \xi_{\Delta}(t)}{1+\xi^{T}(t) \xi(t)} & \text { if } & \left|\varepsilon_{u 1}\right| \geq \bar{\nu}_{0}+\delta \\
0 & \text { if } & \left|\varepsilon_{u 1}\right|<\bar{\nu}_{0}+\delta
\end{array}\right.
\end{aligned}
$$

\subsection{Stability}

The boundedness of all parameter errors has now been shown and it is therefore possible to modify Theorem 3.3 and arrive to the following result.

Theorem 3.4 The system in Eq. (3.26) has bounded solutions for all $\nu_{0} \leq u_{0}$ if

$$
\begin{aligned}
& \text { (i) } \sqrt{V\left(t_{0}\right)} \leq \frac{1}{\sqrt{2\left|k^{*}\right| \lambda_{\max }(\Gamma)}} \frac{q_{0}-\rho \frac{\left|k^{*}\right| r_{0}+\nu_{0}}{\left|u_{0}-\nu_{0}\right|}\left|2 p_{b}\right|\left|\bar{\theta}^{* T} C \|-q_{0}\right|}{\rho \frac{r_{0}}{u_{0}-\nu_{0}}\left|2 p_{b}\right|\left|\bar{\theta}^{* T} C\left\|-q_{0} \mid+2 p_{b}\right\| C \|\right.} \\
& \text { (ii) } \quad x^{T}\left(t_{0}\right) P x\left(t_{0}\right) \leq \lambda_{\min }(P)\left[\frac{2 p_{b}\left|u_{0}-\nu_{0}\right|}{\left|2 p_{b}\right|\left|\bar{\theta}^{* T} C \|-q_{0}\right|}\right]^{2} .
\end{aligned}
$$

Further

$$
x^{T}(t) P x(t) \leq \lambda_{\min }(P)\left[\frac{2 p_{b}\left|u_{0}-\nu_{0}\right|}{\left|2 p_{b}\left\|\bar{\theta}^{* T} C\right\|-q_{0}\right|}\right]^{2} \quad \forall t \geq t_{0} .
$$

Proof: See Appendix A. 


\section{Simulations}

\subsection{1st Order System}

The following adaptive control problem was simulated to study the behavior of solutions for a first order plant. The transfer functions of the plant and the model were chosen to be

$$
W_{p}(s)=\frac{1}{s-1} \quad \text { and } \quad W_{m}(s)=\frac{1}{s+1}
$$

respectively. The controller in Eq. (3.3) in section 2.1 was used with $u_{0}=10$ as the bound on the control signal amplitude and with two adjustable parameters $\theta$ and $k$. The outputs of the plant and the model output as well as the feedback signal $v$ and the control input are shown in Figs. 3-2-3-5 for the cases (a) $r(t) \equiv 0.0$, (b) $r(t) \equiv 5,(\mathrm{c}) r(t)=5 \sin (0.5 t)$ and (d) $r(t)=5 \operatorname{sgn}[\sin (0.5 t)]$. Initial conditions for the plant and the model were chosen as $x_{p}(0)=-9.9, x_{m}(0)=-9.9, \theta(0)=-2.2$ and $k(0)=1.1$. The following observations were made.

- The output error tends asymptotically to zero in all cases.

- The initial conditions chosen in the simulation satisfy conditions (i) and (ii) in Theorem 3.1. It was observed that the system became unstable when the conditions were violated even slightly, for example with $x_{p}(0)=-10.01$.

\subsection{Output Feedback, Relative degree 2}

The following adaptive control problem was simulated to study the behavior of solutions when the relative degree of the plant is greater than 1 . The transfer functions of the plant and the model were chosen to be

$$
W_{p}(s)=\frac{1}{s^{2}-1} \quad \text { and } \quad W_{m}(s)=\frac{1}{s^{2}+2 s+1}
$$

respectively. The controller was implemented as in Eq. (3.16) with the fixed control parameters $\Lambda=-1$ and $l=1$. The true values of the control parameters are then $k^{*}=1, \theta_{1}^{*}=-2, \theta_{0}^{*}=-4$ and $\theta_{2}^{*}=0$. The plant and the model outputs as well as 
the feedback signal $v$ and the control input $u$ are shown in Fig. 3 for the cases of (a) $r(t) \equiv 0.0$, (b) $r(t) \equiv 5$, (c) $r(t)=5 \sin (0.5 t)$ and (d) $r(t)=5 \operatorname{sgn}[\sin (0.5 t)]$. The initial conditions for the plant and the model were chosen as $y_{p}(0)=-5$ and $y_{m}(0)=$ -5 , and the control parameter vector $\theta(0)=1.1 \theta^{*}$. The following observations were made.

- The output error tends asymptotically to zero in all cases except in case (d) where the input saturates periodically.

- The input saturates for a sufficiently small interval in the initial transients during which time the plant is stabilized. However, the initial conditions exceeded the bounds specified in conditions (i) and (ii) in Theorem 3.3 showing that these conditions are somewhat conservative. As in the simulation study in section 3.1, instability did result when the magnitude of the initial conditions was increased further. 
Plant and Model Outputs

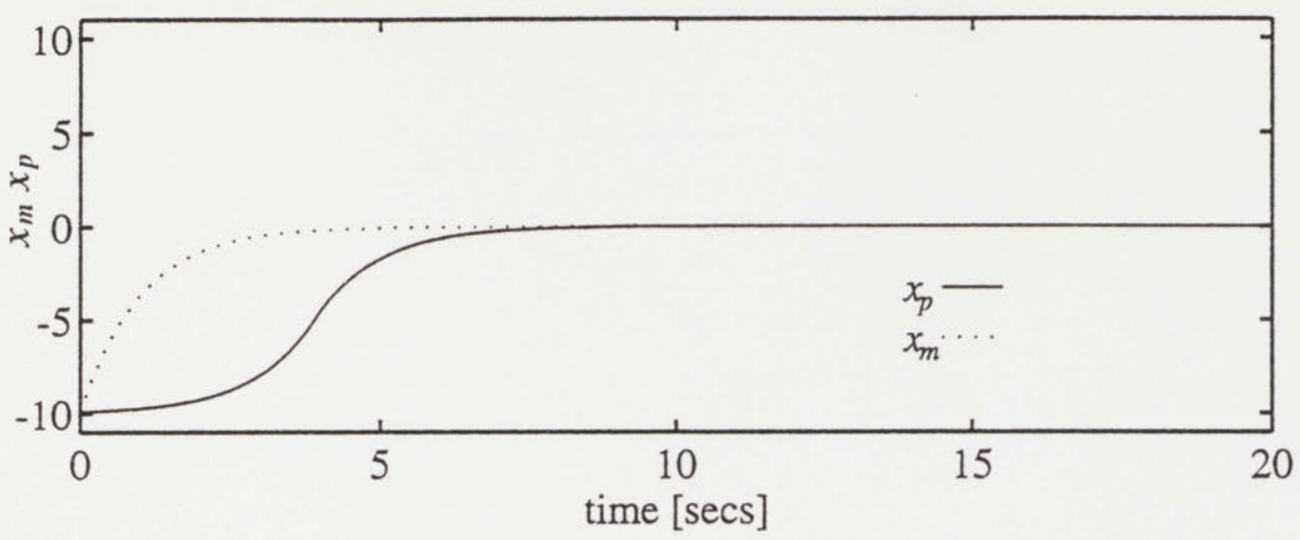

Control Signals

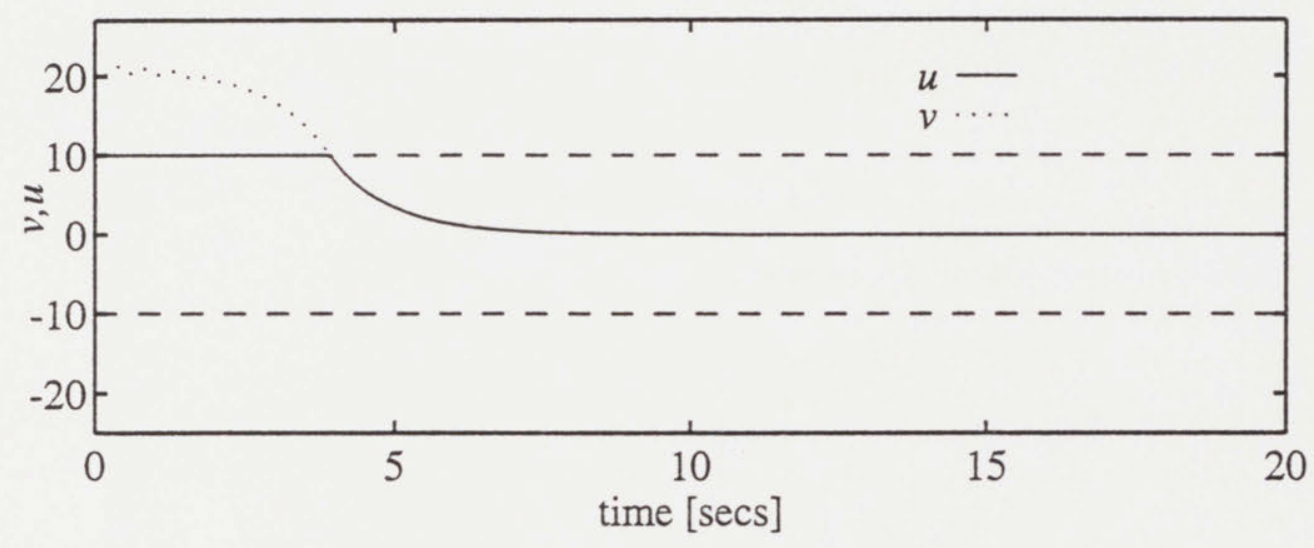

Parameter Errors

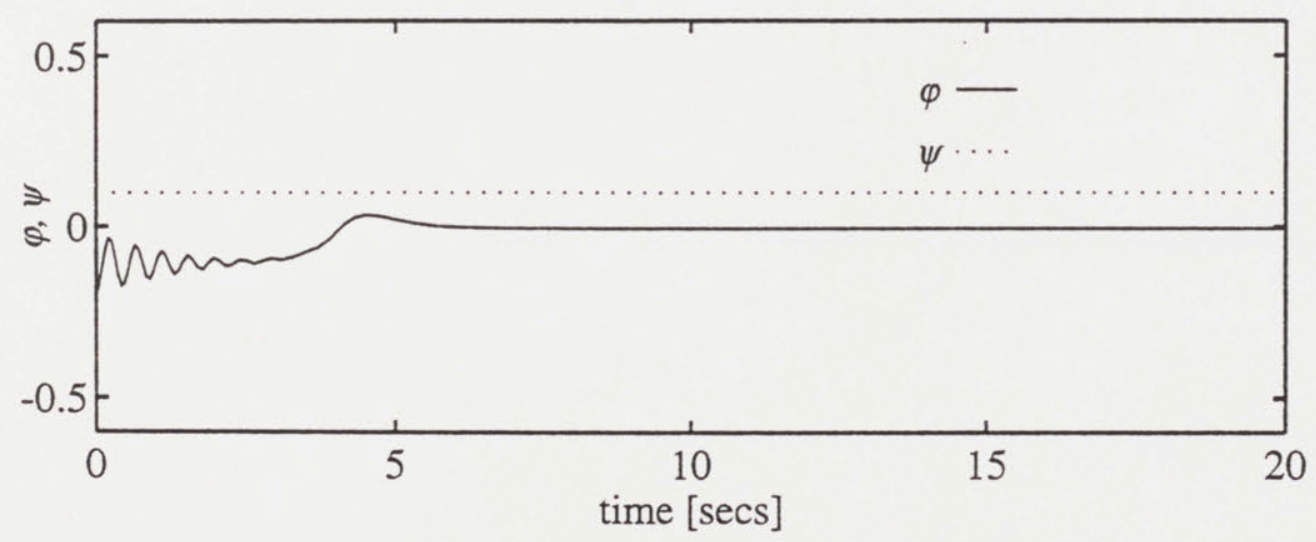

Figure 3-2: Case (a) for Adaptive Control of a 1st-order Continuous-Time Plant. Simulation results for case (a) when the reference input is $r(t) \equiv 0$. The plots show the time history of the plant and model outputs, the control signal before and after saturation, and parameter errors. 
Plant and Model Outputs

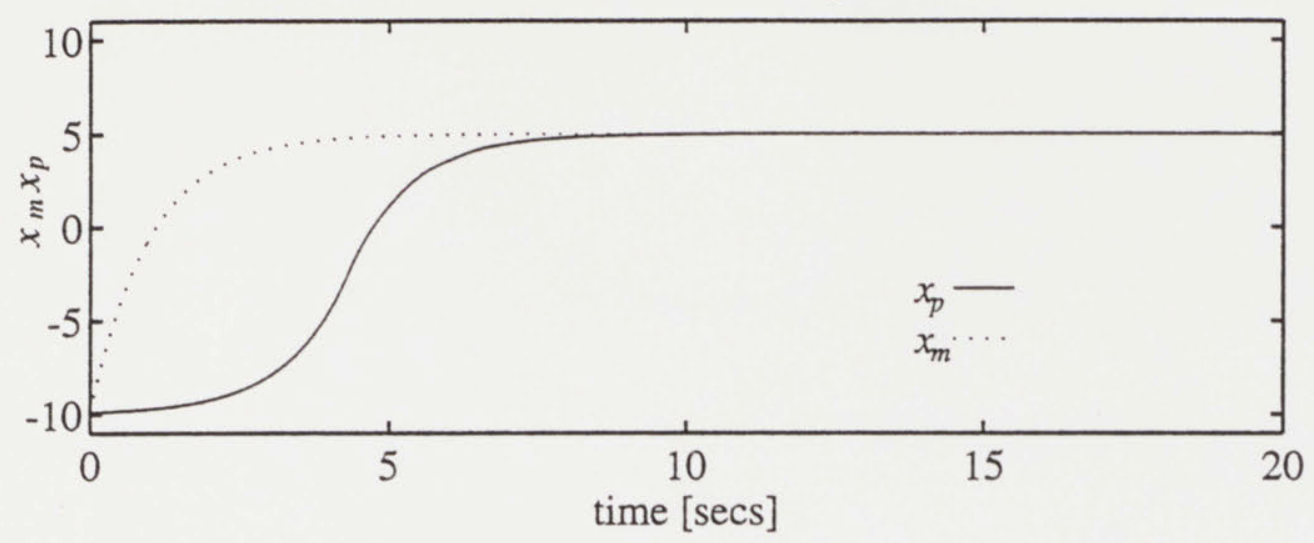

Control Signals

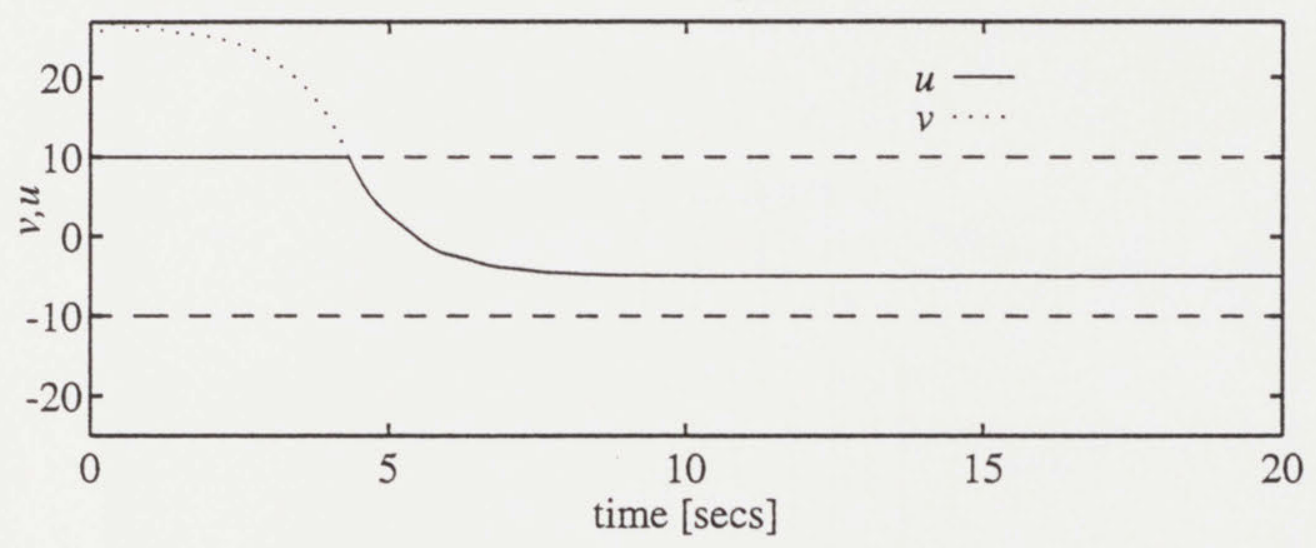

Parameter Errors

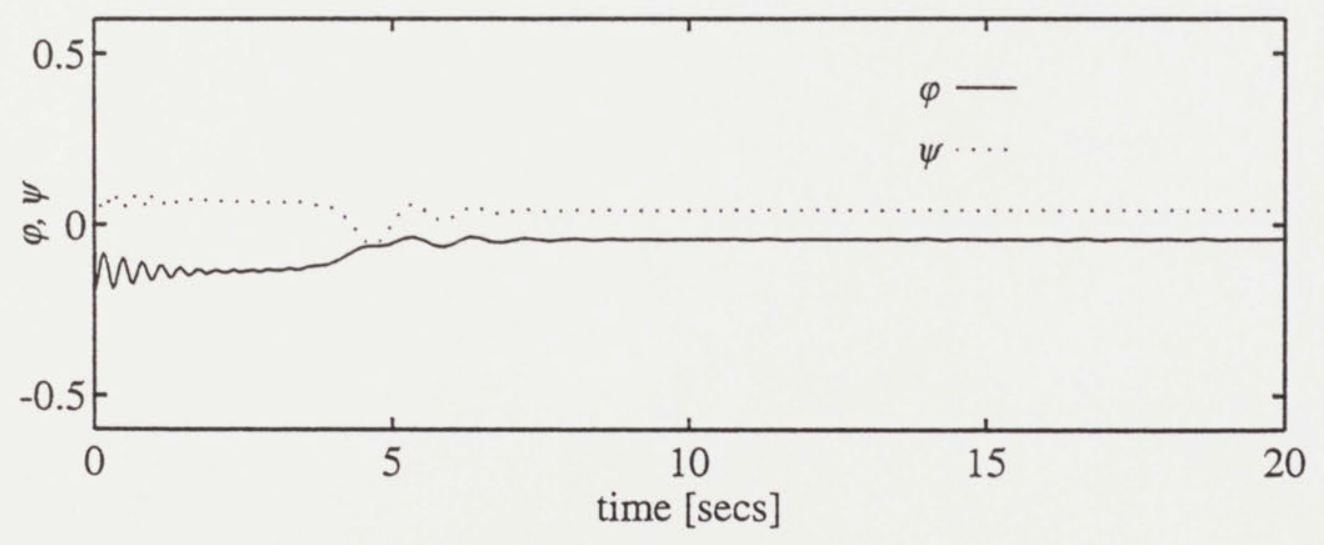

Figure 3-3: Case (b) for Adaptive Control of a 1st-order Continuous-Time Plant. Simulation results for case (b) when the reference input is $r(t) \equiv 5$. The plots show the time history of the plant and model outputs, the control signal before and after saturation, and parameter errors. 
Plant and Model Outputs

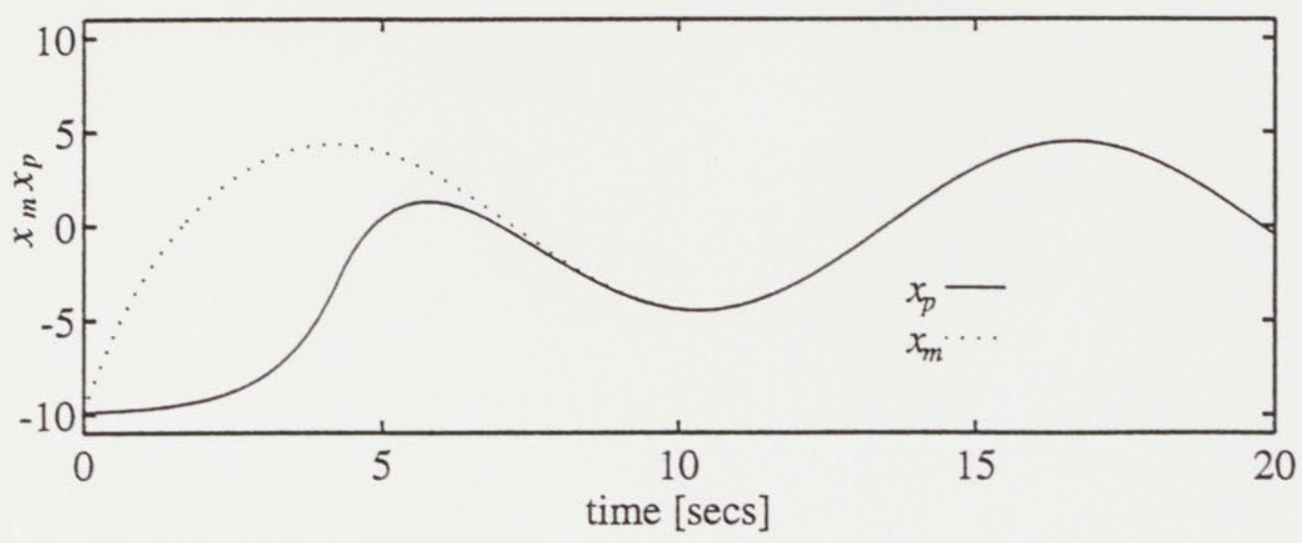

Control Signals

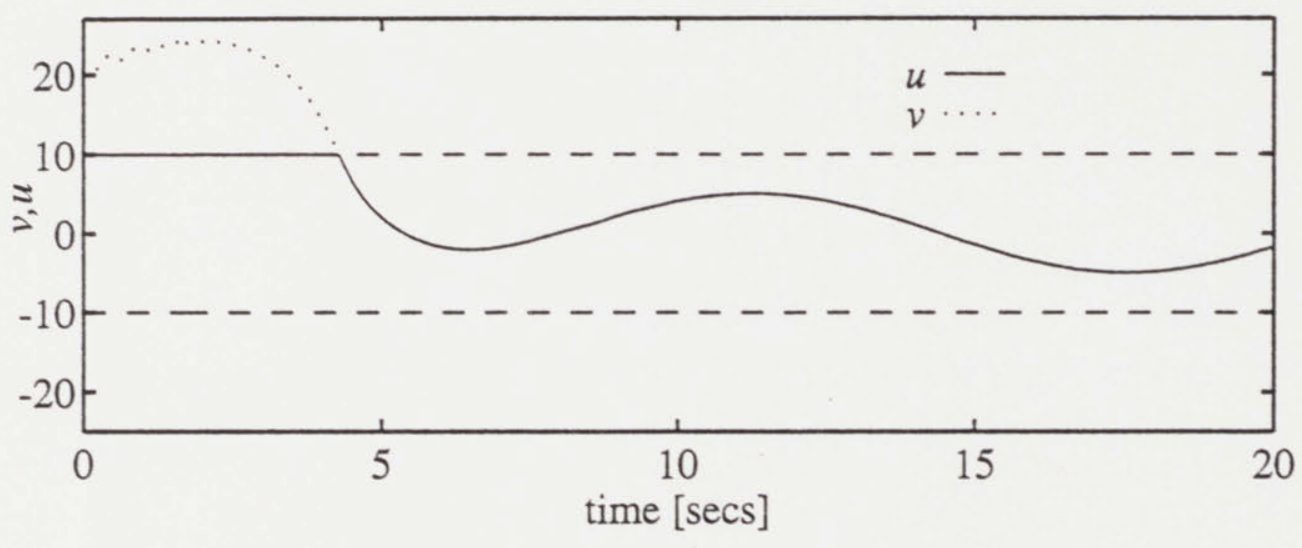

Parameter Errors

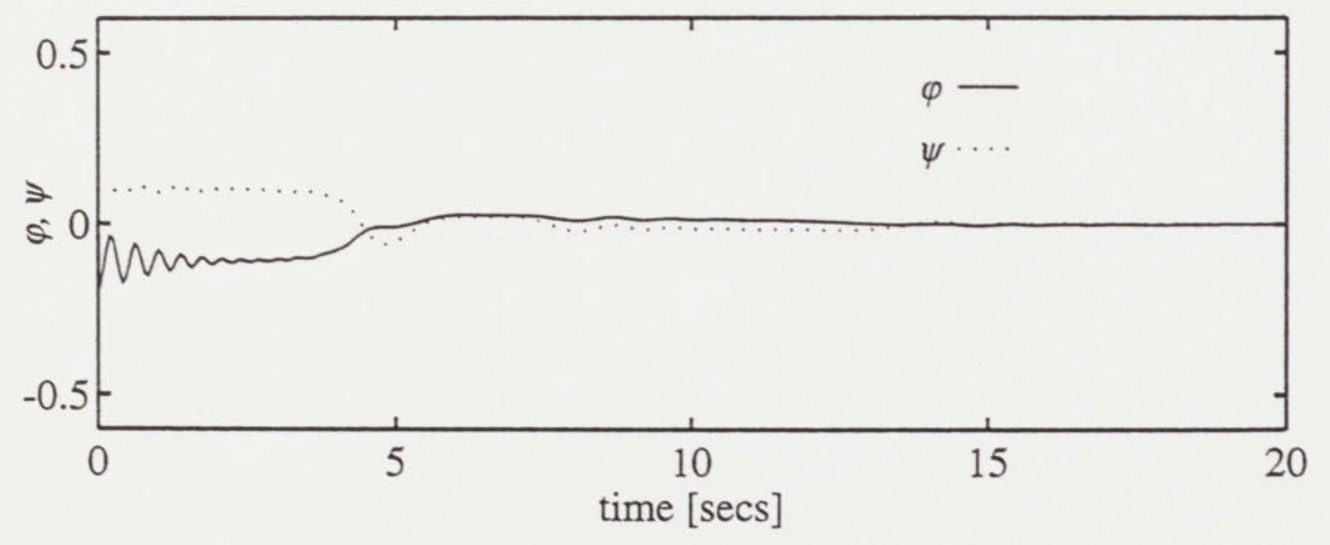

Figure 3-4: Case (c) for Adaptive Control of a 1st-order Continuous-Time Plant. Simulation results for case (c) when the reference input is $r(t)=5 \sin (0.5 t)$. The plots show the time history of the plant and model outputs, the control signal before and after saturation, and parameter errors. 
Plant and Model Outputs

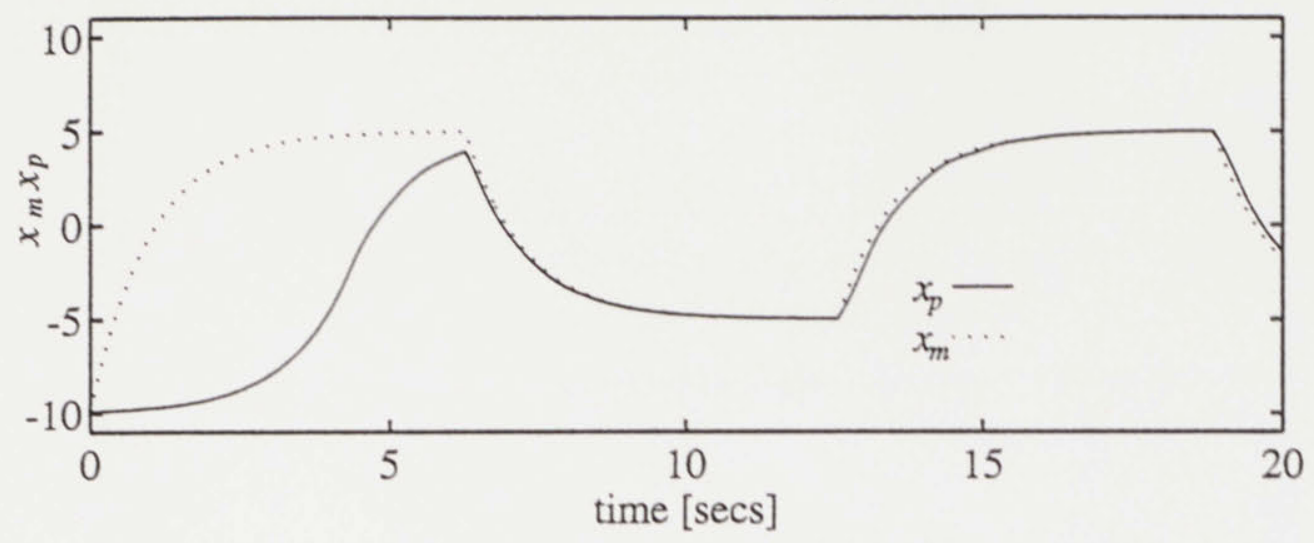

Control Signals

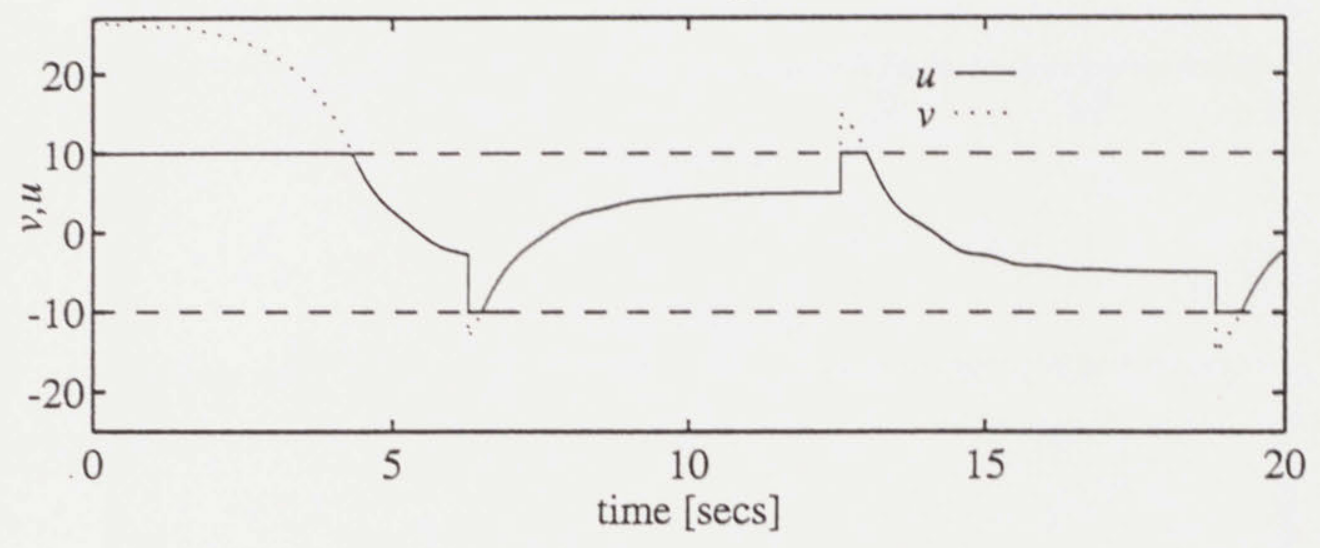

Parameter Errors

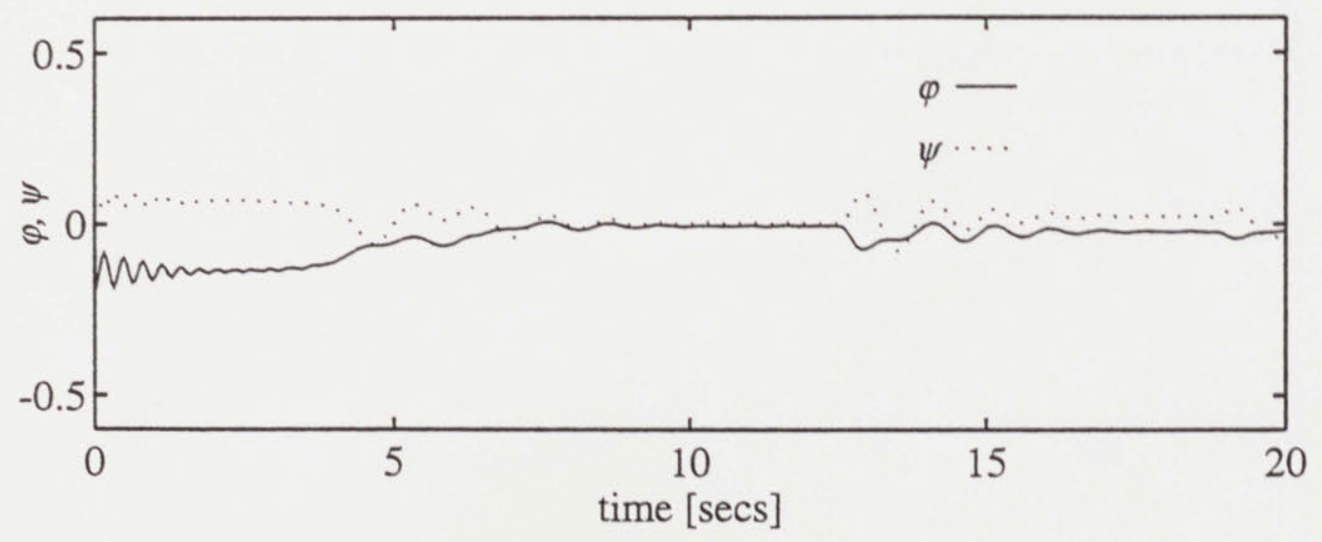

Figure 3-5: Case (d) for Adaptive Control of a 1st-order Continuous-Time Plant. Simulation results for case (d) when the reference input is $r(t)=5 \operatorname{sgn}[\sin (0.5 t)]$. The plots show the time history of the plant and model outputs, the control signal before and after saturation, and parameter errors. 
Plant \& Model Outputs

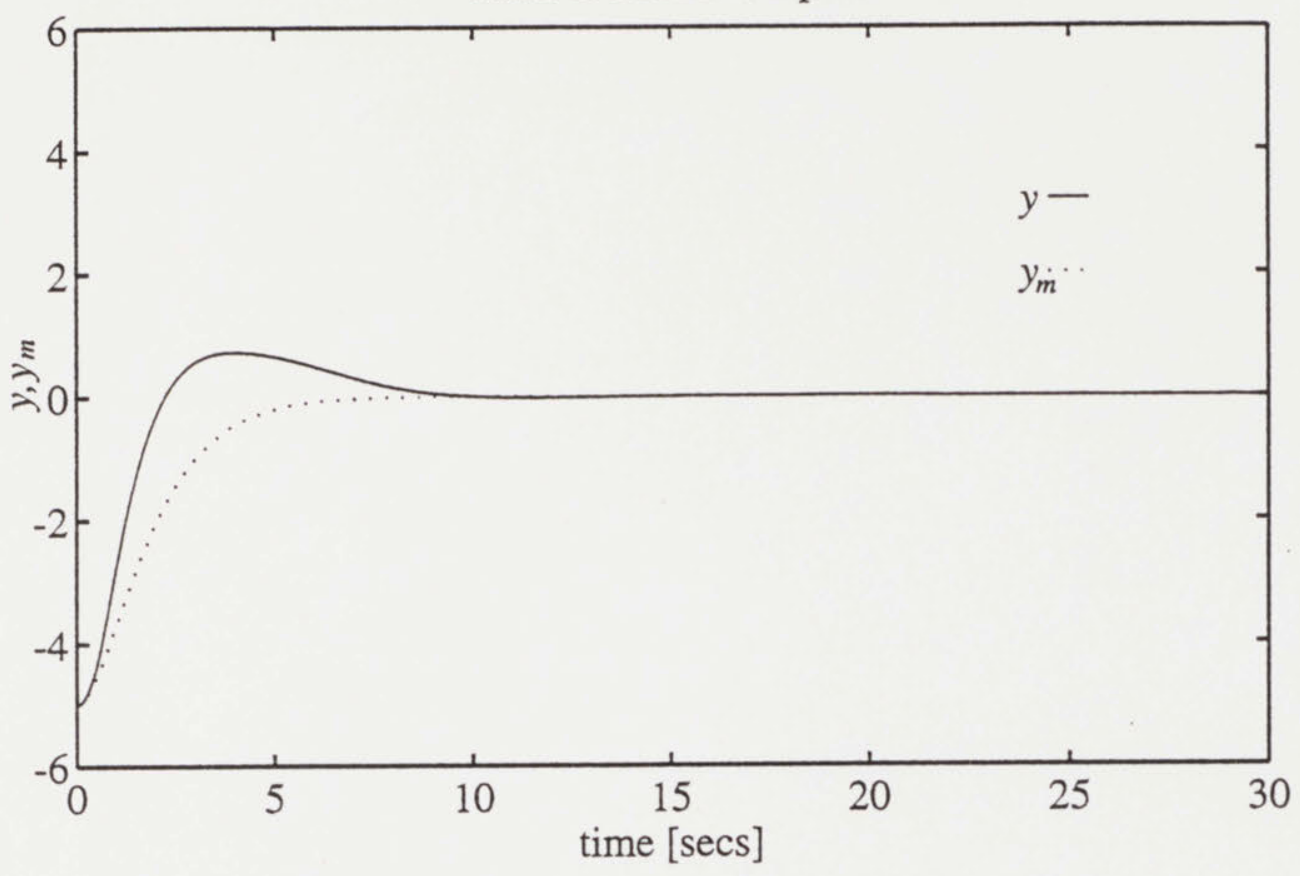

Control Signals

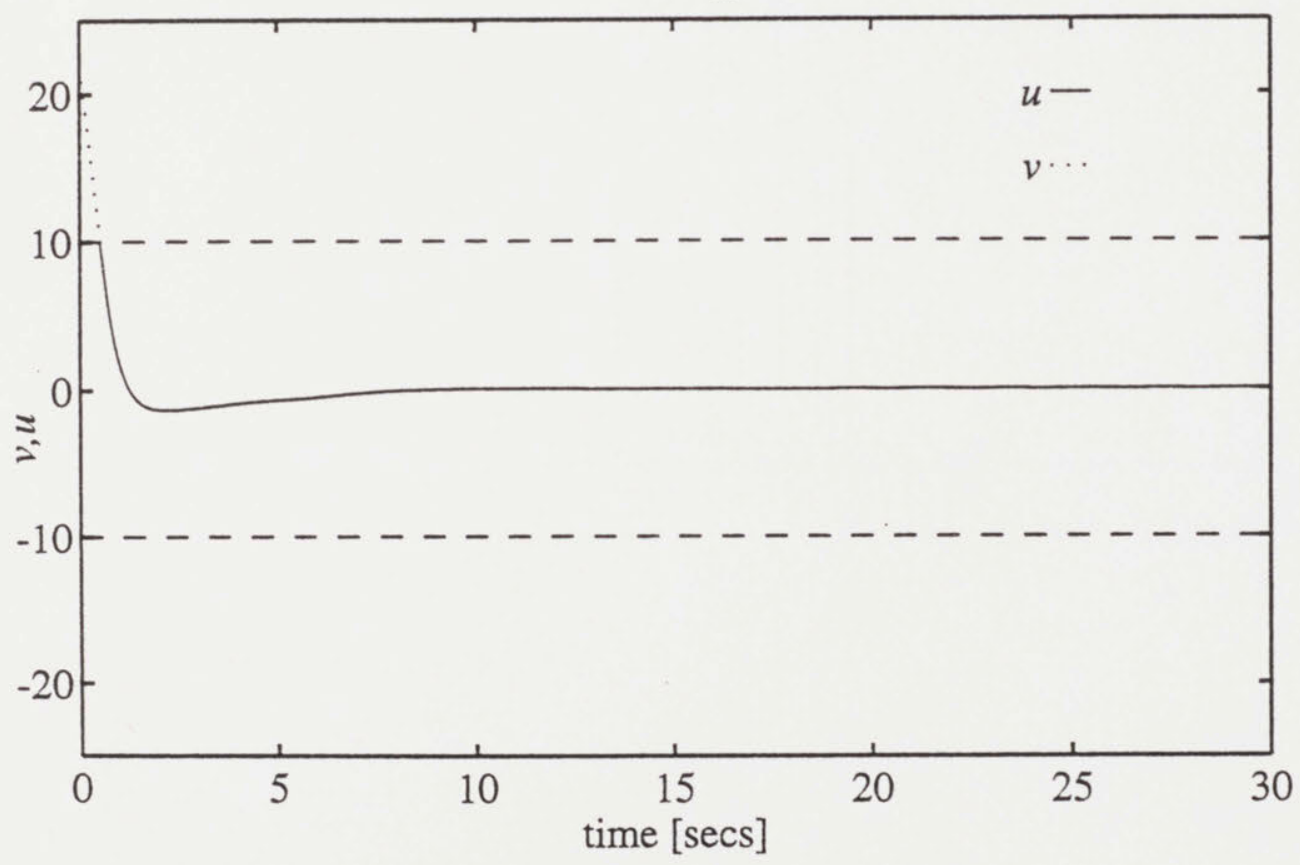

Figure 3-6: Case (a) for Adaptive Control of a 2nd-order Continuous-Time Plant. Simulation results for case (a) when the reference input is $r(t) \equiv 0$. The plots show the time history of the plant and model outputs and the control signal before and after saturation. 
Plant \& Model Outputs

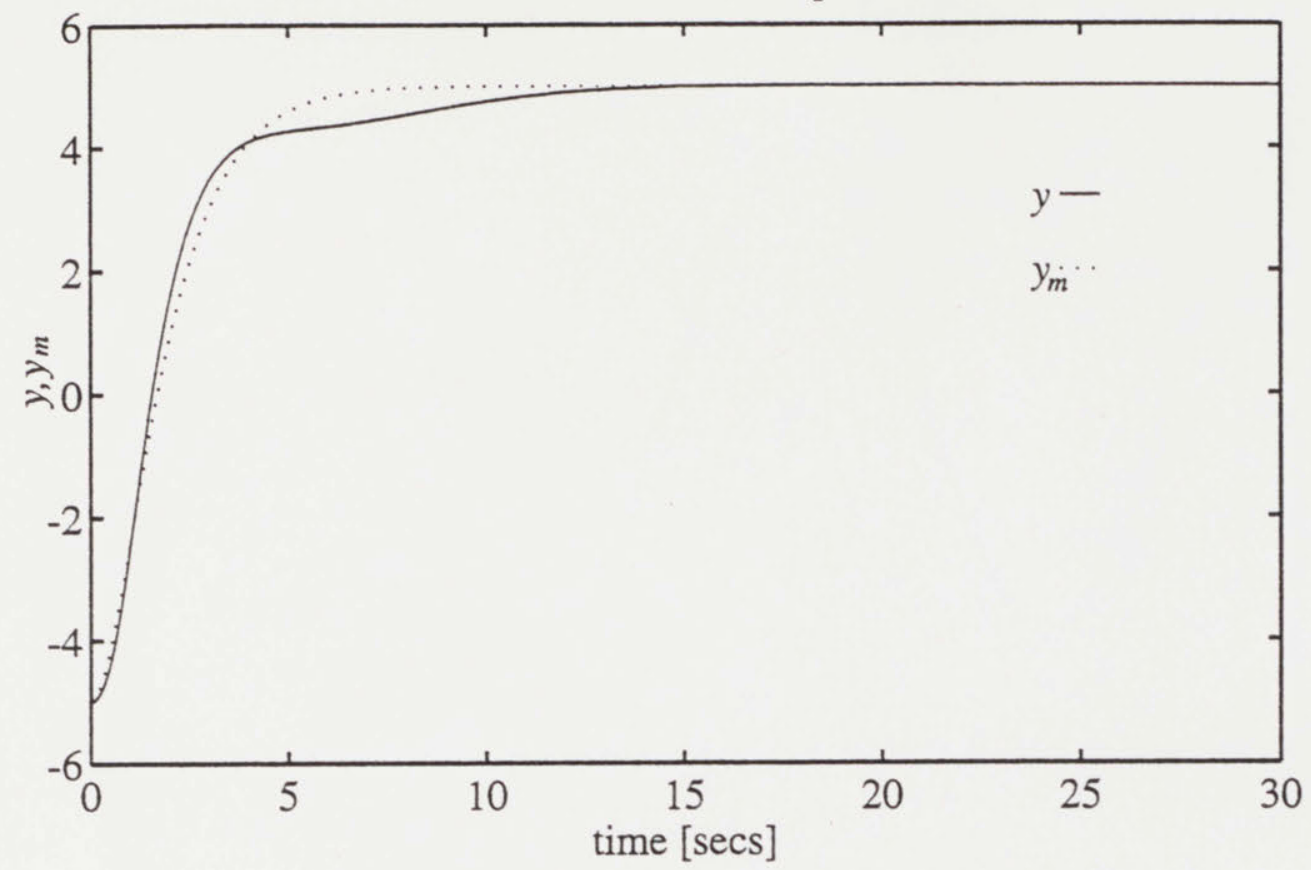

Control Signals

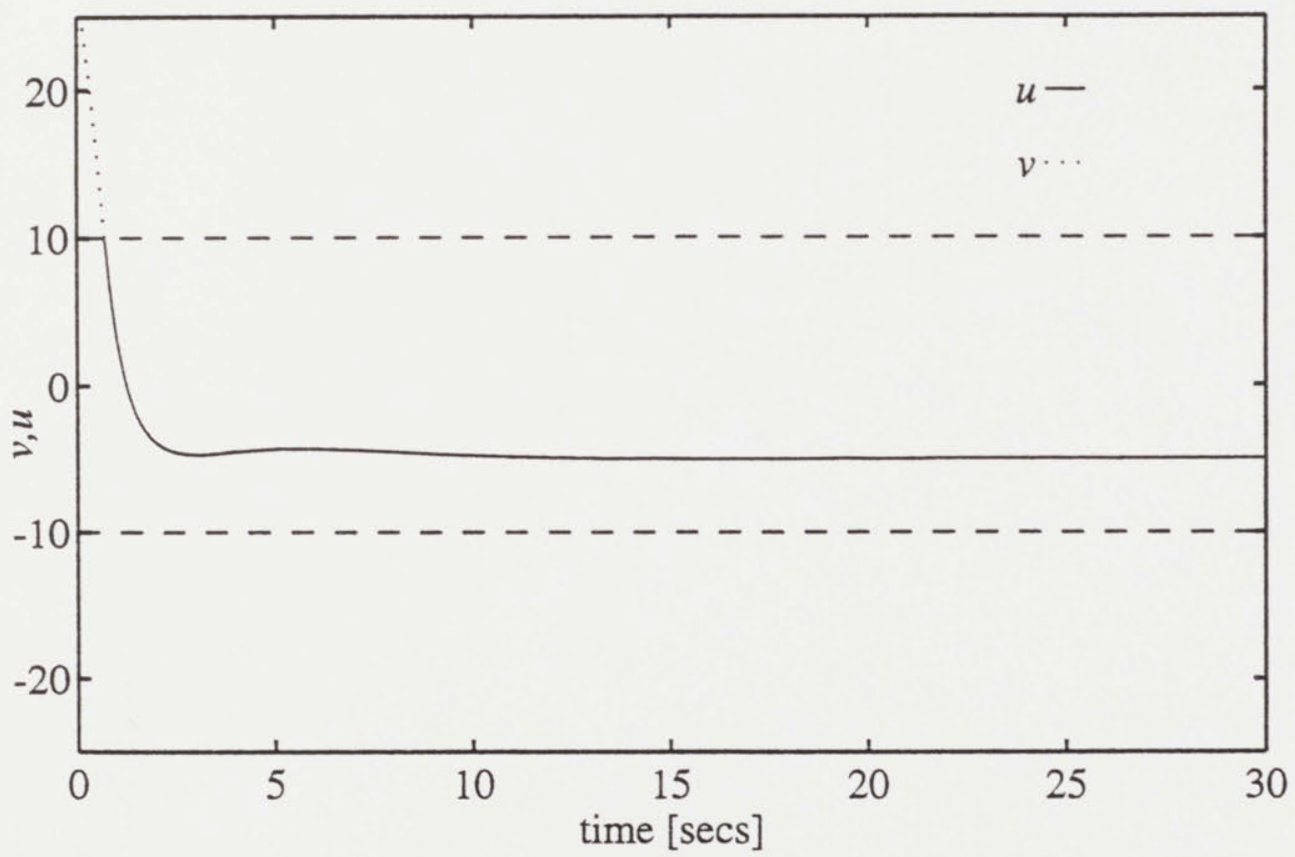

Figure 3-7: Case (b) for Adaptive Control of a 2nd-order Continuous-Time Plant. Simulation results for case (b) when the reference input is $r(t) \equiv 5$. The plots show the time history of the plant and model outputs and the control signal before and after saturation. 
Plant \& Model Outputs

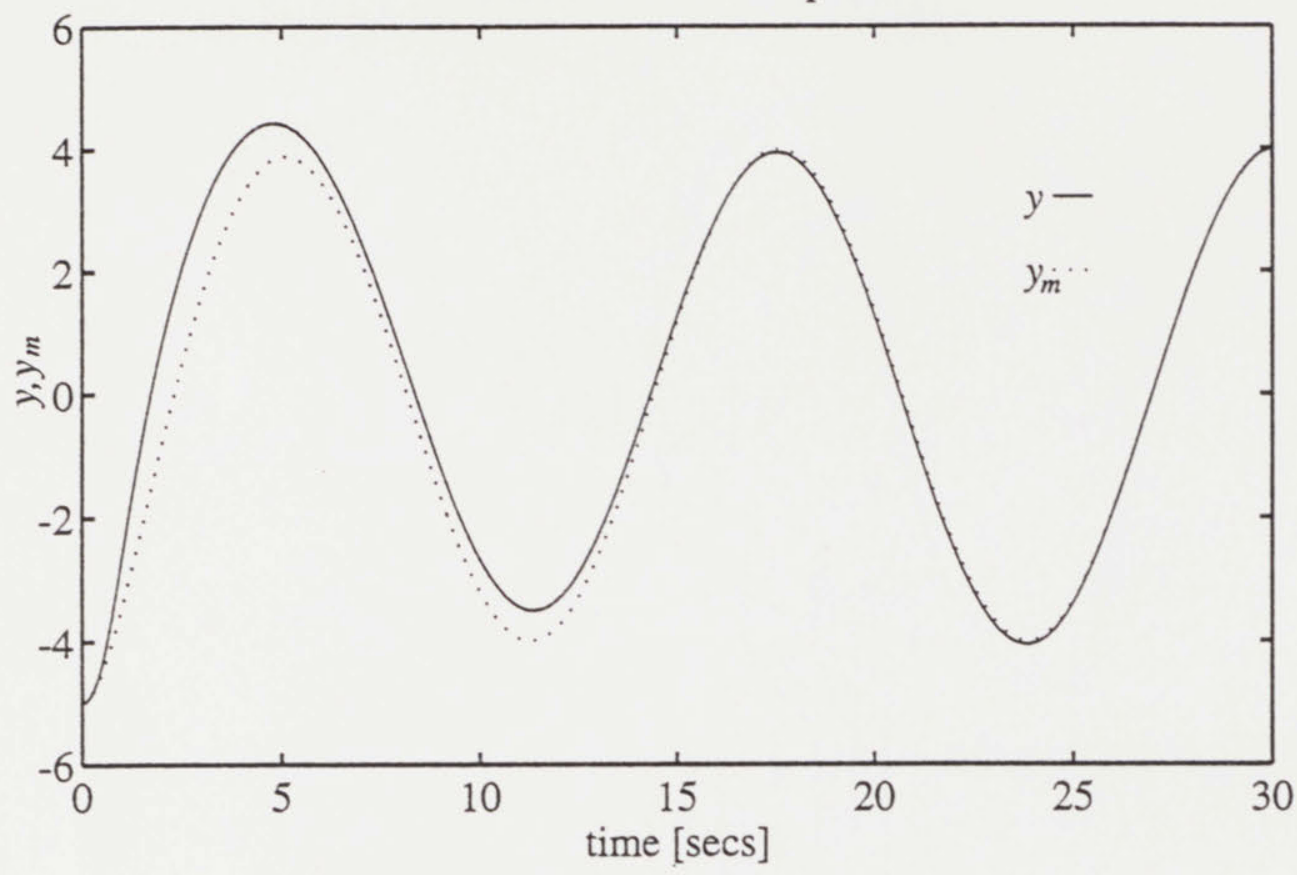

Control Signals

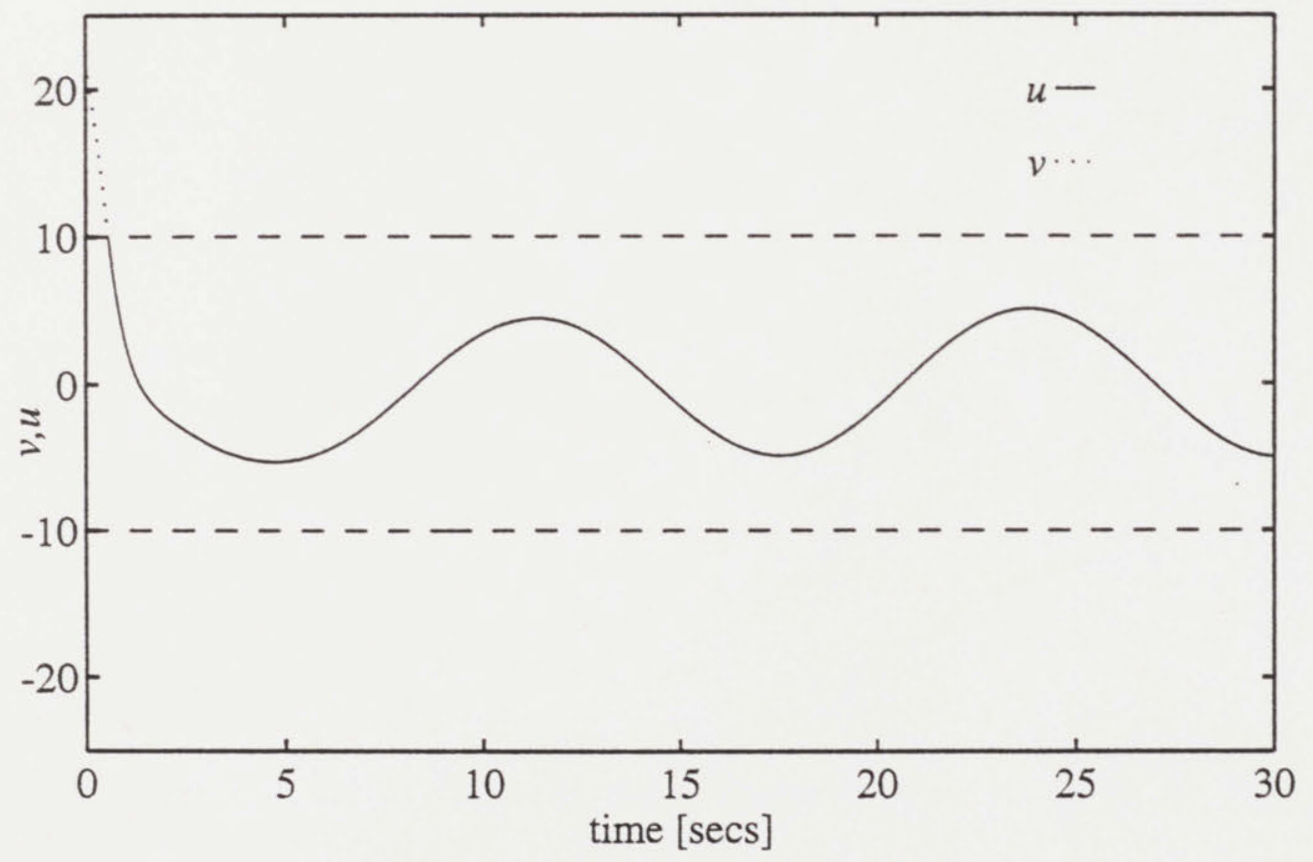

Figure 3-8: Case (c) for Adaptive Control of a 2nd-order Continuous-Time Plant. Simulation results for case (c) when the reference input is $r(t)=5 \sin (0.5 t)$. The plots show the time history of the plant and model outputs and the control signal before and after saturation. 
Plant \& Model Outputs

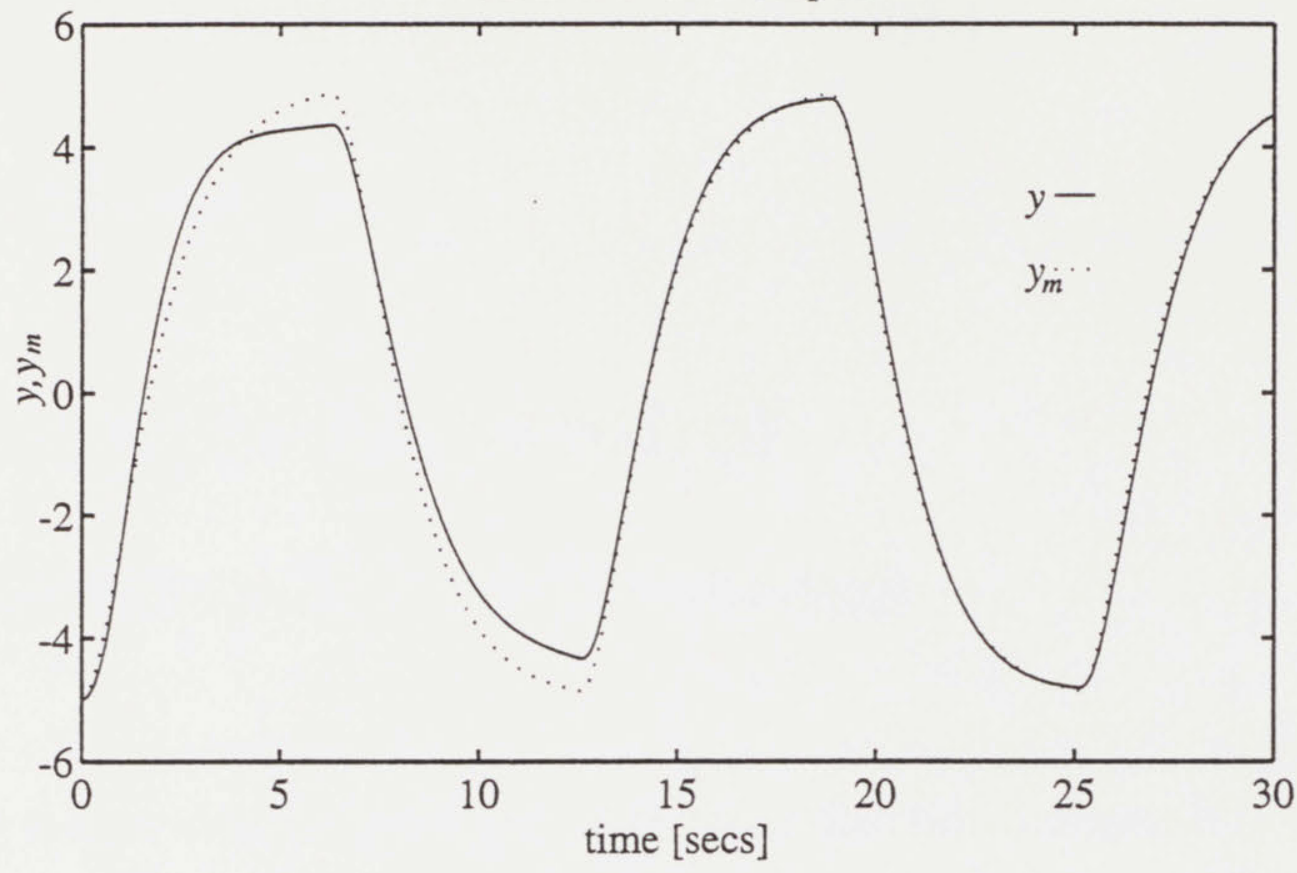

Control Signals

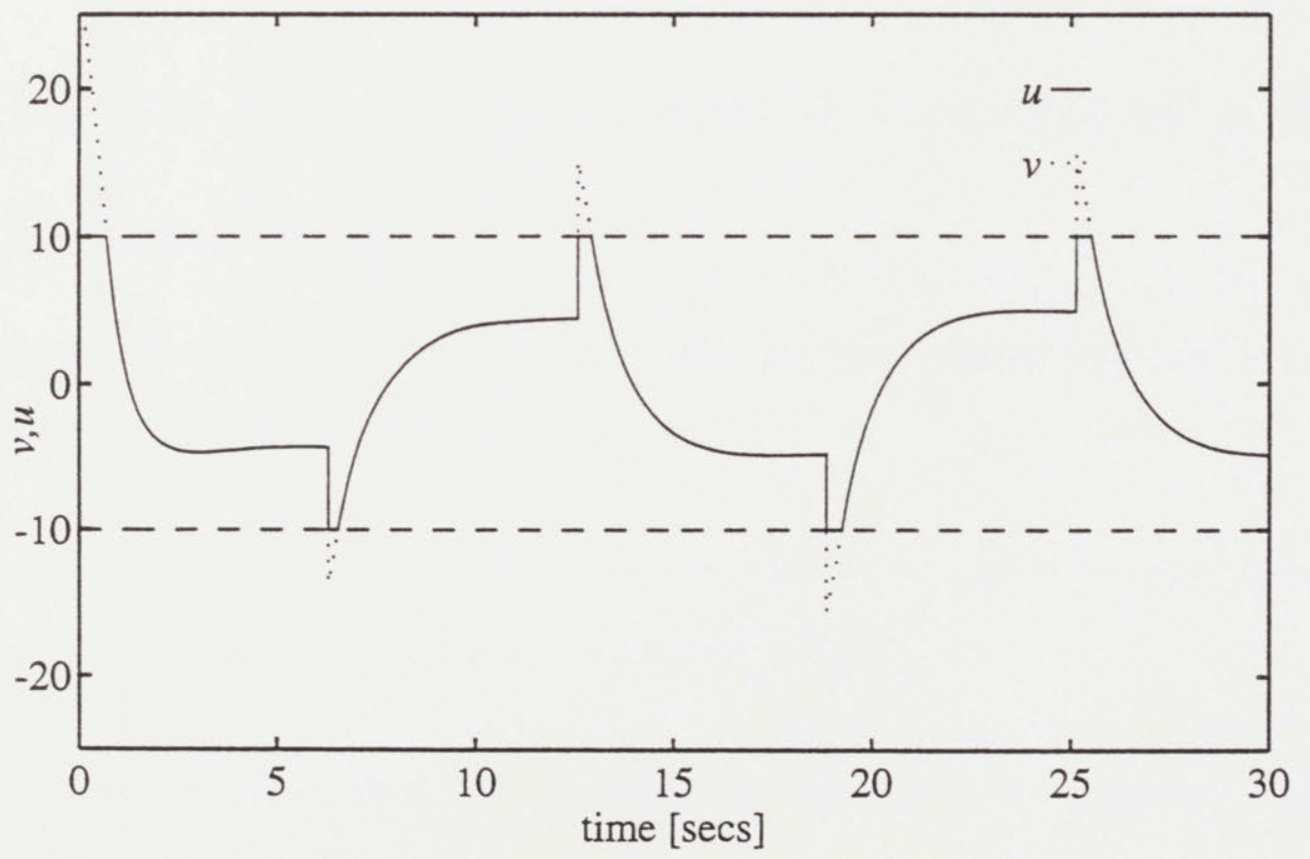

Figure 3-9: Case (d) for Adaptive Control of a 2nd-order Continuous-Time Plant. Simulation results for case (d) when the reference input is $r(t)=5 \operatorname{sgn}[\sin (0.5 t)]$. The plots show the time history of the plant and model outputs and the control signal before and after saturation. 


\section{Chapter 4}

\section{Adaptive Control of \\ Discrete-Time Systems}

In this chapter some new results on the problems described in chapter 2 in the case of discrete-time systems are stated in Theorems 4.1- 4.3 where control schemes and stability of the overall system is established.

\section{Model Reference Adaptive Control of a First Order Plant}

Statement of the Problem: A plant with an input-output pair $\left\{u(\cdot), x_{p}(\cdot)\right\}$ is described by the scalar equation

$$
y(t+1)=a_{p} y(t)+b_{p} u(t)
$$

where $a_{p}$ and $b_{p}$ are unknown, but the sign of $b_{p}$ is assumed to be known. The input $u(t)$ is additionally subject to the magnitude constraint

$$
|u(t)| \leq u_{0}
$$


where $u_{0}$ is a known constant.

A reference model is described by the first-order difference equation

$$
y^{*}(t+1)=a_{m} y^{*}(t)+b_{m} r(t)
$$

where $a_{m}$ is a known constant, $b_{m}$ is known, and $r(t)$ is a piecewise-continuous bounded function whose magnitude is such that

$$
|r(t)| \leq r_{0} .
$$

It is assumed that $a_{m}, b_{m}$ and $r(t)$ have been chosen so that $y^{*}(t)$ represents the output desired of the plant at time $t$. The aim is to determine a bounded control input $u(t)$ so that all signals in the system remain bounded, for a given set of initial conditions, and $y$ tracks $y^{*}$ as closely as possible.

Rewriting the plant equation as

$$
y(t+1)-a_{m} y(t)=\left(a_{p}-a_{m}\right) y(t)+b_{p} u(t)
$$

it follows that a control input of the form

$$
u(t)=-\left[\frac{a_{p}-a_{m}}{b_{p}}\right] y(t)+\frac{1}{b_{p}} b_{m} r(t) \triangleq \theta_{0}^{T} \phi^{\prime}(t)
$$

ensures that $\left|y(t)-y^{*}(t)\right| \rightarrow 0$ as $t \rightarrow \infty$, where

$$
\theta_{0}=\left[\theta_{0 y}, \theta_{0 u}\right]^{T}, \theta_{0 y}=\frac{a_{p}-a_{m}}{b_{p}}, \theta_{0 u}=\frac{1}{b_{p}}, \text { and } \phi^{\prime}(t)=\left[-y(t), y(t+1)-a_{m} y(t)\right]^{T} \text {. }
$$

Obviously, since $\theta_{0}$ is unknown, Eq. (4.2) cannot be implemented. However, from Eq. (4.1), the control input can be expressed as

$$
u(t)=\theta_{0}^{T} \bar{\phi}(t)
$$


where

$$
\bar{\phi}(t)=\left[-y(t), y(t+1)-a_{m} y(t)\right]^{T} .
$$

Using the predictor form in Eq. (4.4), $\theta_{0}$ can be estimated using the well known parameter estimation scheme [3]

$$
\theta(t)=\theta(t-1)+\frac{a \bar{\phi}(t-1)}{c+\bar{\phi}^{T}(t-1) \bar{\phi}(t-1)}\left[u(t-1)-\bar{\phi}^{T}(t-1) \theta(t-1)\right]
$$

where $0<a<2$ and $c>0$ are constants, which ensures that the norm of the parameter estimate error $\|\tilde{\theta}(t)\| \triangleq\left\|\theta(t)-\theta_{0}\right\|$ is nonincreasing with time so the parameter estimate, $\theta(t)$ is bounded for all $t \geq t_{0}$ (see Lemma B.1).

Once it has been obtained that the control parameter is bounded, the stability of the overall adaptive system is established provided the state variables of the plant and the controller are shown to be remain bounded in the presence of constrained plant inputs. The adaptive controller is chosen as

$$
\begin{aligned}
& u(t)=\left\{\begin{array}{ccc}
v(t) & \text { if } & |v| \leq u_{0} \\
u_{0} \operatorname{sgn}(v(t)) & \text { if } & |v|>u_{0}
\end{array}\right. \\
& v(t)=\theta^{T}(t) \phi^{\prime}(t)
\end{aligned}
$$

with the parameter $\theta(t)$ adjusted as in Eq. (4.6). It will be shown in the next section that the adaptive system defined by Eqs. (4.1), (4.6), and (4.7) has bounded solutions for all initial conditions within a compact set.

\subsection{Stability}

If the plant is asymptotically stable, it immediately follows that for any conditions of the plant, $y_{p}$ is bounded. As a result, $\bar{\phi}(t)$ and hence $v(t)$ are bounded, which ensures the global boundedness of the overall system. The question is whether the same can be said for plants that are not necessarily stable. The following theorem addresses this issue. 
Theorem 4.1 The system defined by Eqs. (4.1) (with $\left|a_{p}\right|>1$ ), (4.6), and (4.7) has bounded solutions if

$$
\begin{aligned}
& \text { (i) }\left\|\tilde{\theta}\left(t_{0}\right)\right\| \leq \frac{\left(1-\left|a_{m}\right|\right)-\left|\frac{b_{m}}{b_{p}}\right| \frac{r_{0}}{u_{0}}\left(\left|a_{p}\right|-1\right)}{\frac{\left|b_{m}\right| r_{0}}{u_{0}}\left(\left|a_{p}\right|-1\right)+\left|b_{p}\right|} \\
& \text { (ii) }\left|y\left(t_{0}\right)\right| \leq \frac{\left|b_{p} u_{0}\right|}{\left|a_{p}\right|-1}
\end{aligned}
$$

where $\tilde{\theta}(t)=\theta(t)-\theta_{0}$.

Proof: See Appendix B.

\section{One-Step-Ahead Adaptive Control of an $n$th Order Plant}

Now an $n$th order linear time-invariant plant with unknown parameters considered and an adaptive controller developed for control in the presence of input constraints. In the following, the notation used in [3] will be followed to represent the problem. Statement of the Problem: The plant to be controlled is described by

$$
A\left(q^{-1}\right) y(t)=q^{-d} \bar{B}\left(q^{-1}\right) u(t)
$$

where $q^{-1}$ is the backward shift operator, $d$ stands for pure time delay and

$$
\begin{array}{r}
A\left(q^{-1}\right)=1+a_{1} q^{-1}+\cdots+a_{n} q^{-n} \\
B\left(q^{-1}\right)=b_{0}+b_{1} q^{-1}+\cdots+b_{m} q^{-m}
\end{array}
$$

and the coefficients of $A(\cdot)$ and $B(\cdot)$ are assumed to be unknown. The control input is such that $|u(t)| \leq u_{0}$ for all $t \geq t_{0}$ and the objective is for the plant output $y(t)$ follow some desired trajectory $y^{*}(t)$ as closely as possible. The plant-model can be 
rewritten in the form of a $d$-step-ahead predictor as [3]

$$
y(t+d)=\alpha\left(q^{-1}\right) y(t)+\beta\left(q^{-1}\right) u(t)
$$

where

$$
\begin{aligned}
\alpha\left(q^{-1}\right) & =\alpha_{0}+\alpha_{1} q^{-1}+\cdots+\alpha_{n-1} q^{n-1} \\
\beta\left(q^{-1}\right) & =\beta_{0}+\beta_{1} q^{-1}+\cdots+\beta_{m+d-1} q^{m+d-1} \\
\beta_{0} & =b_{0} \neq 0
\end{aligned}
$$

To get a linear control form, Eq. (4.8) is expressed as

$$
\begin{aligned}
\frac{1}{\beta_{0}} y(t+d) & =\frac{1}{\beta_{0}} \alpha\left(q^{-1}\right) y(t)+\frac{1}{\beta_{0}} \beta\left(q^{-1}\right) u(t) \\
& =\alpha^{\prime}\left(q^{-1}\right) y(t)+\left[\beta^{\prime}\left(q^{-1}\right)+1\right] u(t)
\end{aligned}
$$

where $\alpha\left(q^{-1}\right)=\beta_{0} \alpha^{\prime}\left(q^{-1}\right)$ and $\beta\left(q^{-1}\right)=\beta_{0}\left[1+\beta^{\prime}\left(q^{-1}\right)\right]$. This makes it possible to rewrite Eq. (4.8) as

$$
u(t)=\bar{\phi}^{T}(t) \theta_{0}
$$

where

$$
\begin{aligned}
\theta_{0} & =\left[\theta_{0 y}^{T}, \theta_{0 u}^{T}\right]^{T}, \quad \theta_{0 y}=\left[\alpha_{0}^{\prime}, \ldots, \alpha_{n-1}^{\prime}\right]^{T}, \theta_{0 u}=\left[\beta_{1}^{\prime}, \ldots, \beta_{m+d-1}^{\prime}\right]^{T}, \theta_{0 r}=\left[\frac{1}{\beta_{0}}\right] \\
\bar{\phi}(t) & =[-y(t), \ldots,-y(t-n+1),-u(t-1), \ldots,-u(t-m-d+1), y(t+d)]^{T} \\
\alpha_{i}^{\prime} & =\frac{\alpha_{i}}{\beta_{0}}, \quad i=1, \ldots, n-1 \\
\beta_{j}^{\prime} & =\frac{\beta_{j}}{\beta_{0}}, \quad j=1, \ldots, m+d-1
\end{aligned}
$$

Is is noted that Eq. (4.9) holds regardless of how the control is obtained and is just a reparametrization of the plant-model. If $\theta_{0}$ was known, then a control of the form

$$
u(t)=\phi^{\prime}(t) \theta_{0}
$$


where

$$
\phi^{\prime T}(t)=\left[-y(t), \ldots,-y(t-n+1),-u(t-1), \ldots,-u(t-m-d+1), y^{*}(t+d)\right],
$$

would ensure that $y(t)$ tends to the desired trajectory $y^{*}(t)$. Since $\theta_{0}$ is unknown and the control is subjected to saturation, the following control input is used instead of Eq. (4.10).

$$
\begin{aligned}
& v(t)=\phi^{\prime}(t) \theta(t) \\
& u(t)=\left\{\begin{array}{ccc}
v(t) & \text { if } & |v(t)| \leq u_{0} \\
u_{0} \operatorname{sgn}(v(t)) & \text { if } & |v(t)|>u_{0}
\end{array}\right.
\end{aligned}
$$

where $\theta(t)$ is a parameter estimate adjusted according to the adaptive law

$$
\theta(t)=\theta(t-1)+\frac{a \bar{\phi}(t-d)}{c+\bar{\phi}^{T}(t-d) \bar{\phi}(t-d)}\left[u(t-d)-\bar{\phi}^{T}(t-d) \hat{\theta}(t-1)\right]
$$

where $0<a<2, \quad c>0$ and the estimate of $\theta_{0 r}$ is kept away from zero by using standard projection methods. From lemma B.1, it follows that $\tilde{\theta}(t)=\theta(t)-\theta_{0}$ is always bounded, and that $\|\tilde{\theta}(t)\|$ is a nonincreasing function such that

$$
\|\tilde{\theta}(t)\| \leq\left\|\tilde{\theta}\left(t_{0}\right)\right\|
$$

\subsection{Stability}

As in the previous section, the stability of the closed-loop system follows immediately if the plant is asymptotically stable, for, the boundedness of the plant input $u$ directly leads to boundedness of $\bar{\phi}$, and Lemma B.1 establishes the boundedness of the control parameter $\theta(t)$. Also, the stability result holds globally. Below, it will be shown that the closed-loop system has bounded solutions even for unstable plants. 
Let

$$
\begin{aligned}
\theta^{T}(t) & =\left[\theta_{y}^{T}(t), \theta_{u}^{T}(t), \theta_{r}(t)\right]^{T} \\
\tilde{\theta}^{T}(t)= & {\left[\tilde{\theta}_{y}^{T}(t), \tilde{\theta}_{u}^{T}(t), \tilde{\theta}_{r}(t)\right] } \\
\psi_{y}(t)= & {[-y(t+d-1),-y(t+d-2), \ldots,-y(t-n+1)]^{T} } \\
\psi_{u}(t)= & {[-u(t-1),-u(t-2), \ldots,-u(t-m-d+1)]^{T}, } \\
n_{d}=n+d-1, \quad & m_{d}=m+d-1, P \triangleq \operatorname{diag}\left(n_{d}, n_{d-1}, \ldots, 1\right) \\
\left|y^{*}(t)\right| \leq y_{\max }, \quad & \|\tilde{\theta}(t)\| \leq \tilde{\theta}_{\max } \\
\tilde{\theta}_{y}(t)= & \theta_{y}(t)-\theta_{0 y}, \tilde{\theta}_{u}(t)=\theta_{u}(t)-\theta_{0 u}, \quad \tilde{\theta}_{r}(t)=\theta_{r}(t)-\theta_{0 r} .
\end{aligned}
$$

Let $\|\cdot\|_{1} \triangleq \sum|\cdot|$. The main result is stated in the following theorem.

Theorem 4.2 The adaptive system defined by Eqs. (4.8) and (4.11) has bounded solutions if

(i) $\tilde{\theta}_{\max } \leq \frac{\left(1-\left\|\theta_{0 u}\right\|_{1}\right)-n_{d} \frac{y_{\max }^{*}}{\left|\beta_{0}\right| u_{0}}\left(\left|\beta_{0}\right|\left\|\theta_{0 y}\right\|-\frac{1}{\sqrt{n_{d}}}\right)}{\sqrt{n_{d}}\left|\beta_{0}\right|\left(1-\left\|\theta_{0 u}\right\|_{1}\right)+n_{d}\left(\sqrt{m_{d}}+\frac{y_{\max }^{*}}{u_{0}}\right)\left(\left|\beta_{0}\right|\left\|\theta_{0 y}\right\|-\frac{1}{\sqrt{n_{d}}}\right)}$

(ii)

$$
\psi_{y}^{T}\left(t_{0}-d+1\right) P \psi_{y}\left(t_{0}-d+1\right) \leq\left[\frac{u_{0}\left(1-\left\|\theta_{0 u}\right\|_{1}\right)}{\left\|\theta_{0 y}\right\|-\frac{1}{\sqrt{n_{d}}\left|\beta_{0}\right|}}\right]^{2}
$$

where $P=\operatorname{diag}\left[n_{d},\left(n_{d}-1\right), \ldots, 2,1\right]$ is an $n_{d} \times n_{d}$ positive-definite matrix.

Proof: See Appendix B.

\section{Remarks:}

1. It should be noted that the one-step-ahead controller analyzed here was chosen because of its analytical simplicity and not because of its performance which is questionable in many situations. 
2. Theorems 4.1-4.3 are equally valid for any adaptive law which ensures bounded parameter estimates. For example, if a least-squares adaptive law is used, the theorems will be unchanged provided that the saturation effect is properly accounted for [3].

3. Theorem 4.2 has been shown to hold for a specific choice of $P$ as shown in the theorem statement. It is however believed that the theorem can be stated more generally, similar to Theorems 3.2-3.4, without specifying $P$ except that it is a solution to the discrete Lyapunov equation, $A_{m}^{T} P A_{m}-P=-Q$ where $Q$ is some positive definite matrix. This specific choice of $P$ simplifies both the conditions and the proofs of the theorems, making the problem more tractable.

\section{Control in the presence of Bounded Distur- bances}

The stability results for discrete-time systems given in Theorems 4.1 and 4.2 can, similarly to the continuous-time case, readily be extended to include bounded disturbances. Here only the case of one-step-ahead control of a plant of arbitrary order will be treated as the modifications needed for Theorem 4.1 follow quite trivially. Assume , as in the continuous-time case, that a disturbance $\nu(t)$, where $|\nu(t)| \leq \nu_{0}$, enters the system, between the limiter and the plant (see Fig 3-1). The $\nu$ term denotes a bounded "noise" that can account for for disturbances from many different sources, and is not limited to input disturbances. Computer round-off error, unmodelled dynamics, as well as measurement noises can be cast into this form.

The actual input then becomes

$$
u_{d}(t)=u_{c}(t)+\nu(t)
$$

and system as described as d-step-ahead predictor in Eq (4.8) without disturbance, 
becomes with disturbances

$$
y(t+d)=\alpha\left(q^{-1}\right) y(t)+\beta\left(q^{-1}\right)\left[u_{c}(t)+\nu(t)\right]
$$

Similar to before the predictor is written in linear control form as

$$
u_{c}(t)=\bar{\phi}_{c}^{T}(t) \theta_{0}+\phi_{\nu}^{T}(t) \theta_{0 u}-\nu(t)
$$

where

$$
\begin{aligned}
\bar{\phi}_{c}^{T}(t) & =\left[-y(t), \ldots,-y(t-n+1),-u_{c}(t-1), \ldots,-u_{c}(t-m-d+1), y(t+d)\right] \\
\phi_{\nu}^{T}(t) & =[-\nu(t-1), \ldots,-\nu(t-m-d+1)] \\
\theta_{0 u}^{T} & =\left[\beta_{1}^{\prime}, \ldots, \beta_{m+d-1}^{\prime}\right] .
\end{aligned}
$$

Let $\bar{\nu} \triangleq \phi_{\nu}^{T}(t) \theta_{0 u}-\nu(t)$ and corresponding $\bar{\nu}_{0} \triangleq \nu_{0}\left(1+\left\|\theta_{0 u}\right\|_{1}\right)$. It can be shown that if the following dead-zone modification of the adaptive law is used then $\|\tilde{\theta}(t)\|$ will be nonincreasing [3]. Define

$$
e_{c}(t-1)=u_{c}(t-d)-\bar{\phi}_{c}^{T}(t-d) \hat{\theta}(t-1)
$$

then the modified adaptive law becomes

$$
\hat{\theta}(t)=\hat{\theta}(t-1)+\left\{\begin{array}{ccc}
\frac{a \bar{\phi}_{c}(t-d) e_{c}(t-1)}{c+\bar{\phi}_{c}^{T}(t-d) \bar{\phi}_{c}(t-d)} & \text { if } & \left|e_{c}(t-1)\right|>2 \bar{\nu}_{0} \\
0 & \text { if } & \left|e_{c}(t-1)\right| \leq 2 \bar{\nu}_{0}
\end{array}\right.
$$

Any other scheme that ensures the parameter error is bounded will do but this one is only given as an example.

If the controller is the same as before, or

$$
v(t)=\phi_{c}^{\prime T}(t) \hat{\theta}(t) \quad u_{c}(t)=\left\{\begin{array}{ccc}
v(t) & \text { if } & |v(t)| \leq u_{0} \\
u_{0} \operatorname{sgn}(v(t)) & \text { if } & |v(t)|>u_{0}
\end{array}\right.
$$


where

$$
\phi^{\prime T}(t)=\left[-y(t), \ldots,-y(t-n+1),-u_{c}(t-1), \ldots,-u_{c}(t-m-d+1), y(t+d)^{*}\right],
$$

then the following stability theorem is obtained.

Theorem 4.3 The adaptive system defined by Eqs. (4.8), (4.11) and (4.14) has bounded solutions if

$$
\begin{aligned}
& \text { (i) } \tilde{\theta}_{\max } \leq \frac{1-\left\|\theta_{0 u}\right\|_{1}-\frac{\bar{\nu}_{0}}{u_{0}}-n_{d}\left(\frac{\bar{\nu}_{0}}{u_{0}}+\frac{y_{\max }^{*}}{\left|\beta_{0}\right| u_{0}}\right)\left(\left|\beta_{0}\right|\left\|\theta_{0 y}\right\|-\frac{1}{\sqrt{n_{d}}}\right)}{\sqrt{n_{d}}\left|\beta_{0}\right|\left[1-\left\|\theta_{0 u}\right\|_{1}-\frac{\bar{\nu}_{0}}{u_{0}}\right]+n_{d}\left(\sqrt{m_{d}}+\frac{y_{\max }^{*}}{u_{0}}\right)\left(\left|\beta_{0}\right|\left\|\theta_{0 y}\right\|-\frac{1}{\sqrt{n_{d}}}\right)} \\
& \text { (ii) } \psi_{y}^{T}\left(t_{0}-d+1\right) P \psi_{y}\left(t_{0}-d+1\right) \leq\left[\frac{u_{0}\left(1-\left\|\theta_{0 u}\right\|_{1}\right)}{\left\|\theta_{0 y}\right\|-\frac{1}{\sqrt{n_{d}}\left|\beta_{0}\right|}}\right]^{2}
\end{aligned}
$$

Proof: See Appendix B.

\section{Simulations}

\subsection{Model Reference Control of a 1st Order System}

The following adaptive control problem was simulated to study the behavior of solutions for a first order plant. The continuous-time transfer functions of a sample-andhold system and corresponding model were chosen to be

$$
W_{p}(s)=\frac{1}{s-1} \quad \text { and } \quad W_{m}(s)=\frac{1}{s+1}
$$

respectively. Using a zero order hold and sampling time of $T=0.1 \mathrm{sec}$ equivalent discrete-time transfer functions become [32].

$$
W_{p}(z)=\frac{-0.1052}{z+1.105} \quad W_{m}(z)=\frac{0.09516}{z+0.9048}
$$


. The controller in Eq. (4.7) was implemented with $u_{0}=10$ as the bound on the control signal amplitude. The correct parameters are in this case $\theta_{0}^{T}=[-1.905,-9.508]$. The outputs of the plant and the model output, the feedback signal $v$ and the control input $u(t)$ as well as the parameter estimate errors are shown in Figs. 4-1-4-4 respectively, for the cases (a) $r(t) \equiv 0.0$, (b) $r(t) \equiv 5$, (c) $r(t)=5 \sin (0.5 t)$ and (d) $r(t)=5 \operatorname{sgn}[\sin (0.5 t)]$. Initial conditions for the plant and the model were chosen as $y(0)=-9.9, y_{m}(0)=-9.9, \theta^{T}(0)=[-1.99,-9.932]$. The following observations were made.

- The output error tends asymptotically to zero in all cases except in case (d) where the control saturates periodically even after the parameter estimates have converged to the correct values.

- The initial conditions chosen in the simulation satisfy conditions (i) and (ii) in Theorem 4.1. It was observed that the system became unstable when the conditions were violated even slightly, for example with $y(0)=-10.01$.

\subsection{One-Step-Ahead Control of a 2nd Order System}

The following adaptive control problem was simulated to study the behavior of solutions for a sample-and-hold system when the order of the plant is greater than 1 . The continuous-time transfer functions of the plant was chosen to be

$$
W_{p}(s)=\frac{-10}{s^{2}+25 s-10}=\frac{-10}{(s+25)(s-0.4)} \text {. }
$$

Using a zero-order hold with a sampling time $T=0.33 \mathrm{~s}$ the equivalent discrete-time transfer function becomes

$$
W_{p}(z)=\frac{B(z)}{A(z)}=\frac{-0.1231 z-0.01793}{z^{2}-1.141 z+2.098110^{-3}}
$$

The controller was implemented as in Eq. (4.11). The true value of the control parameter vector is then $\theta_{0}^{T}=[-9.269,0.002421,0.1456,-8.121]$. The plant and the desired outputs, the feedback signal $v$ and the control input $u$ as well as the norm of 
the parameter error vector are shown in Figs. 4-5-4-8 for the cases of (a) $y^{*}(t) \equiv 0.0$, (b) $y^{*}(t) \equiv 0.8480$, (c) $y^{*}(t)=0.8480 \sin (0.5 t)$ and (d) $y^{*}(t)=0.8480 \operatorname{sgn}[\sin (0.5 t)]$. The initial conditions for the plant and the model were chosen as $y(0)=1.713$, $y(-1)=0$ and the control parameter vector $\theta(0)^{T}=[-9.358,0.002444,0.147,-8.199]$.

- The output error tends asymptotically to zero in all cases except in case (d) where the control saturates periodically.

- The input saturates for a sufficiently small interval in the initial transients during which time the plant is stabilized. and also periodically for case (d). The initial conditions satisfy conditions (i) and (ii) in Theorem 4.2. As in the simulation study in section 4.2 , instability did result when the magnitude of the initial conditions was increased further. 
Plant \& Model Outputs

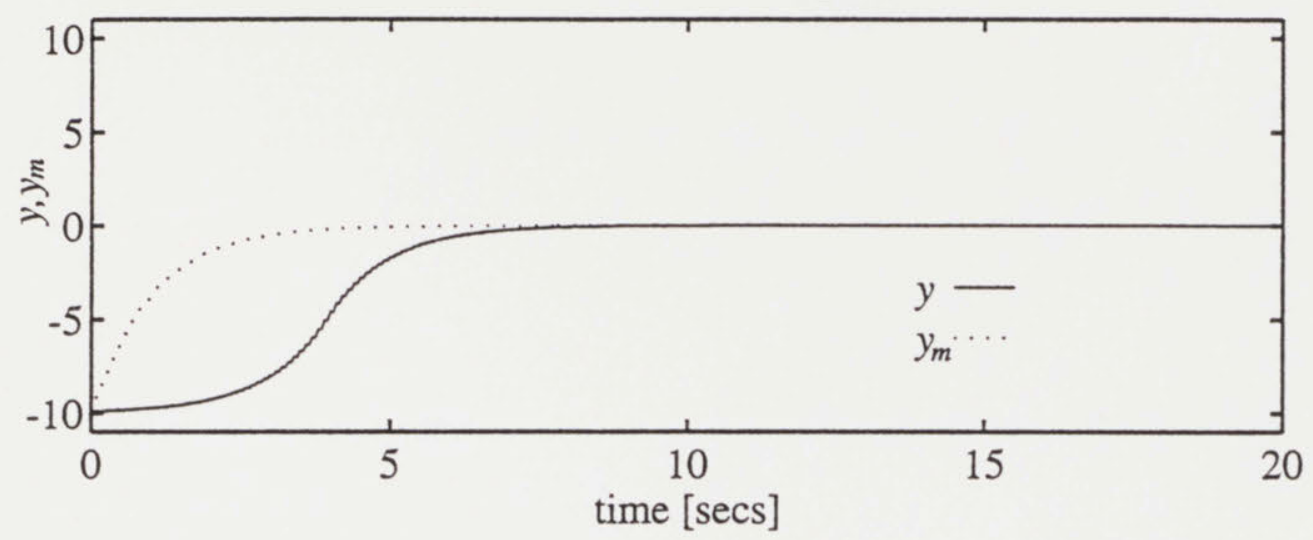

Control Signals

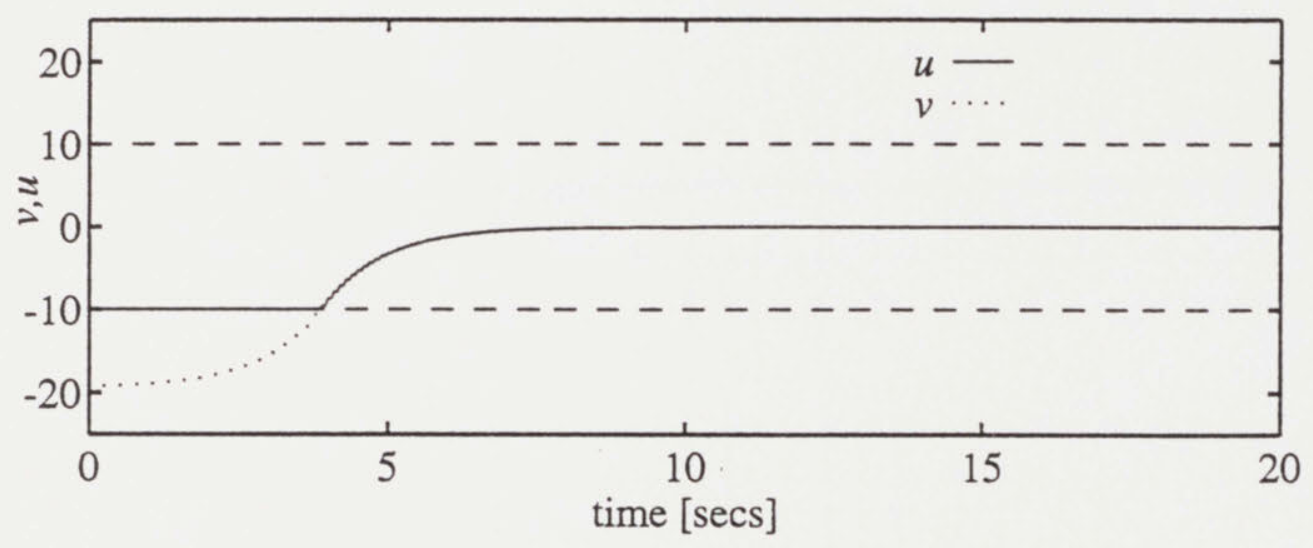

Parameter Errors

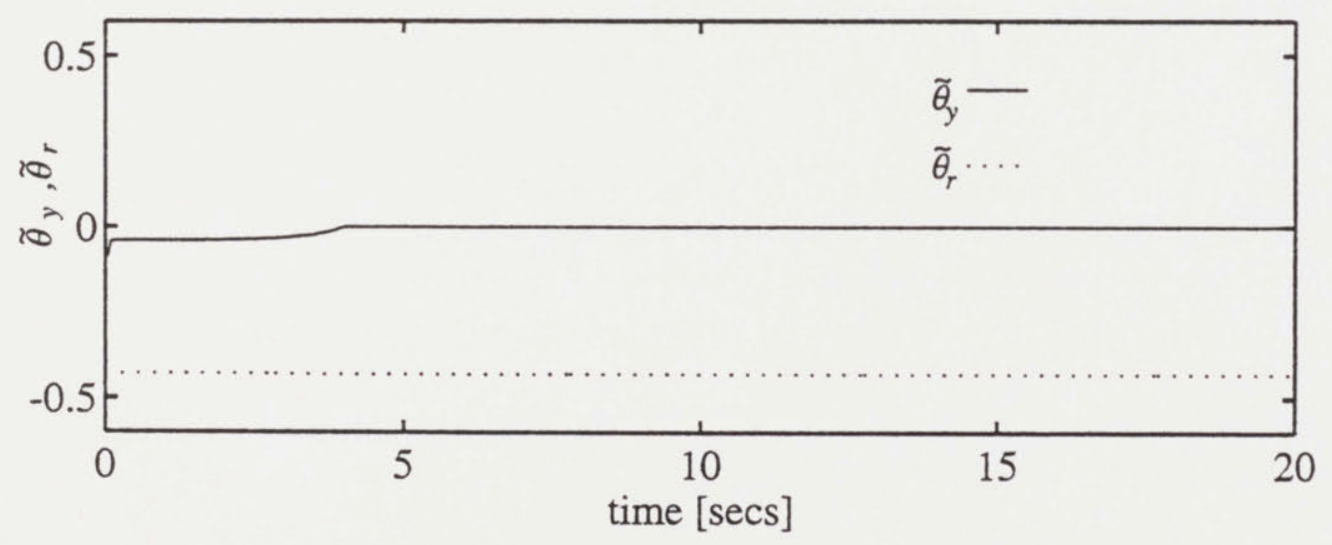

Figure 4-1: Case (a) for Adaptive Control of a 1st-order Discrete-Time Plant. Simulation results for case (a) when the reference input is $r(t) \equiv 0$. The plots show the time history of the plant and model outputs, the control signal before and after saturation, and parameter errors. 
Plant \& Model Outputs

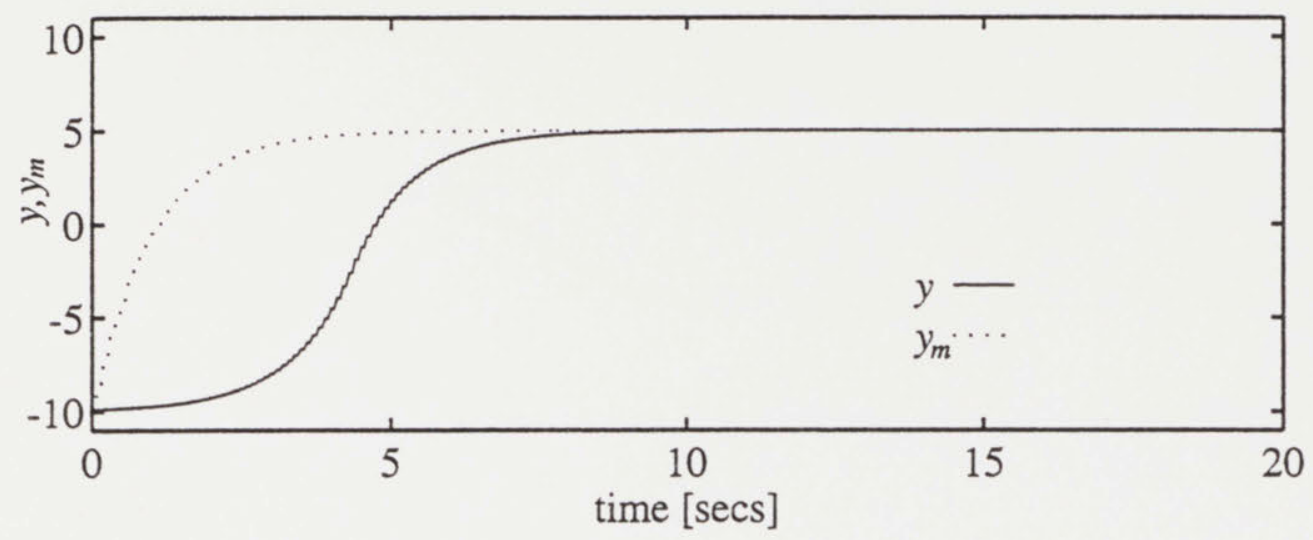

Control Signals

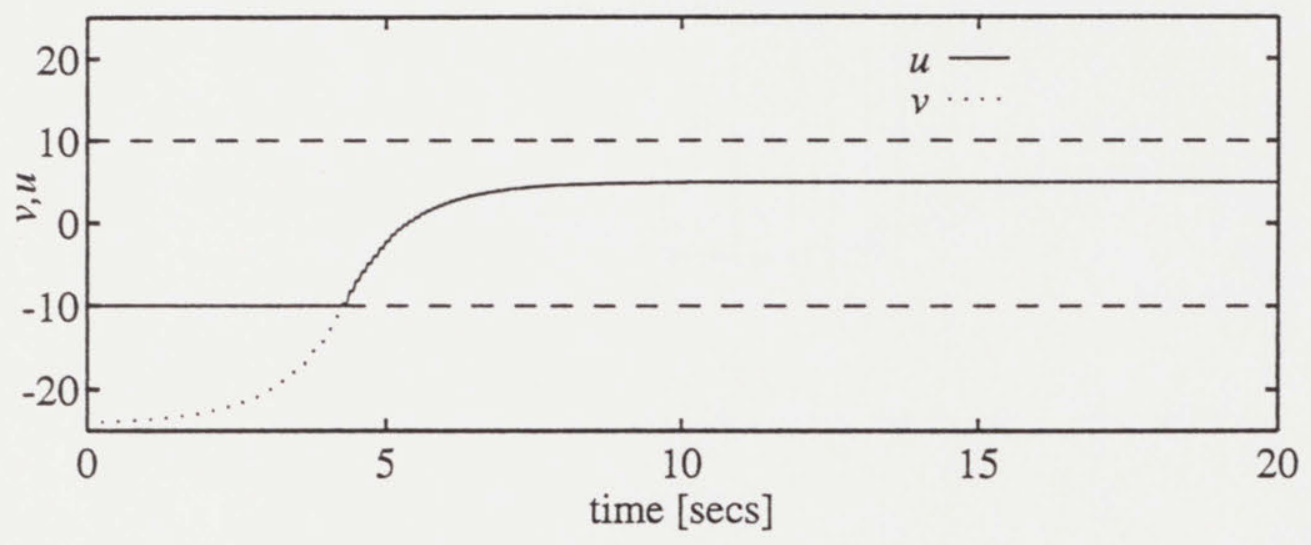

Parameter Errors

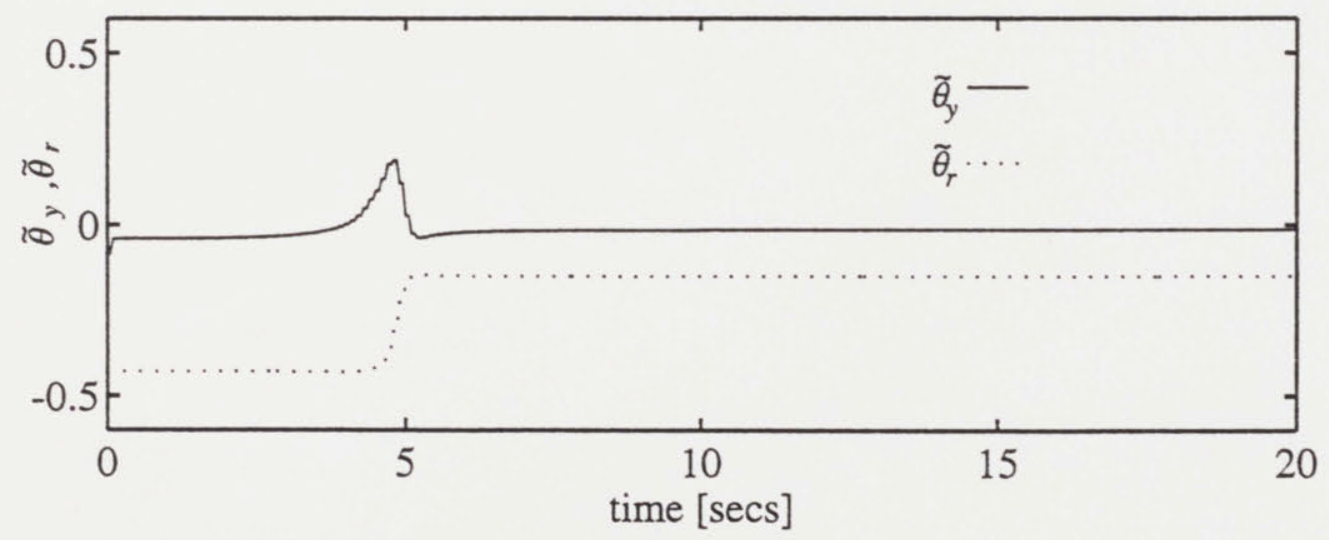

Figure 4-2: Case (b) for Adaptive Control of a 1st-order Discrete-Time Plant. Simulation results for case (b) when the reference input is $r(t) \equiv 5$. The plots show the time history of the plant and model outputs, the control signal before and after saturation, and parameter errors. 


\section{Plant \& Model Outputs}

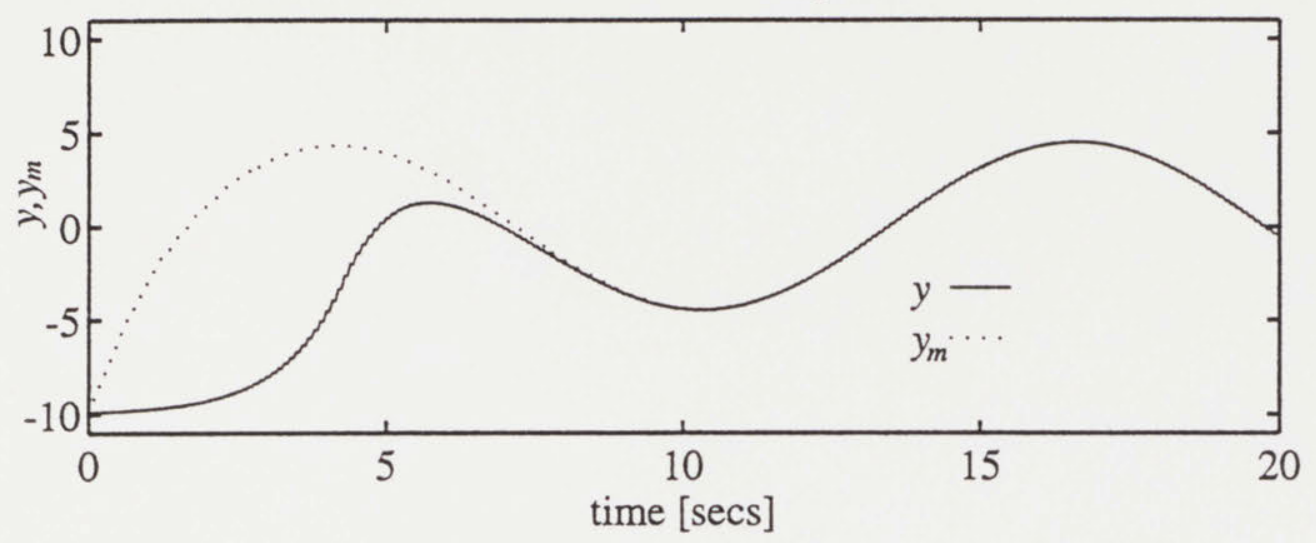

Control Signals

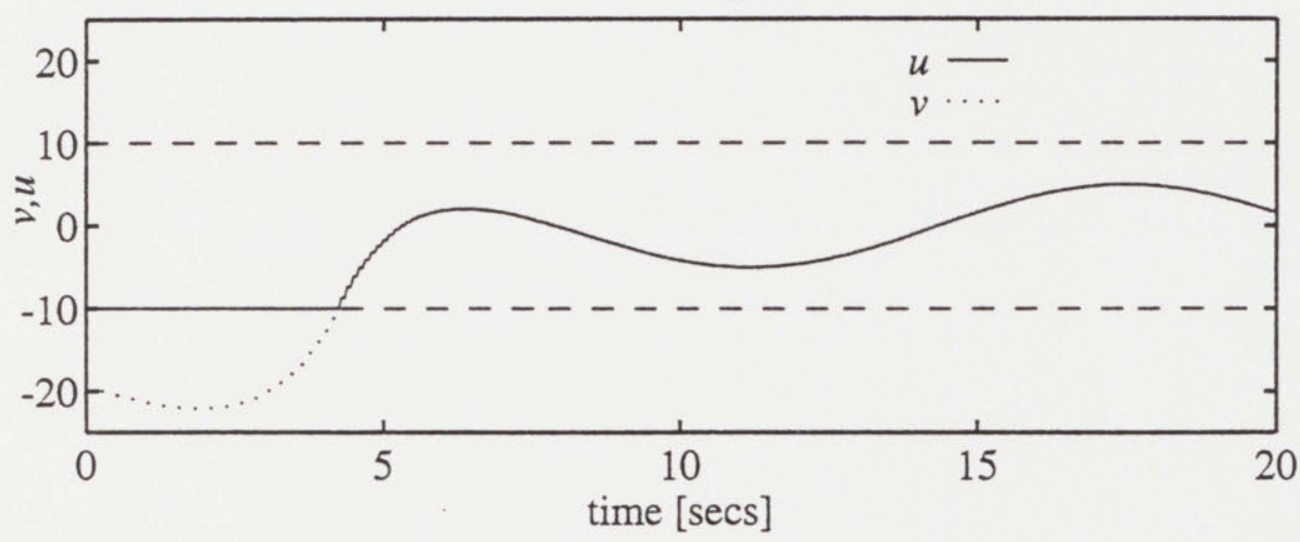

Parameter Errors

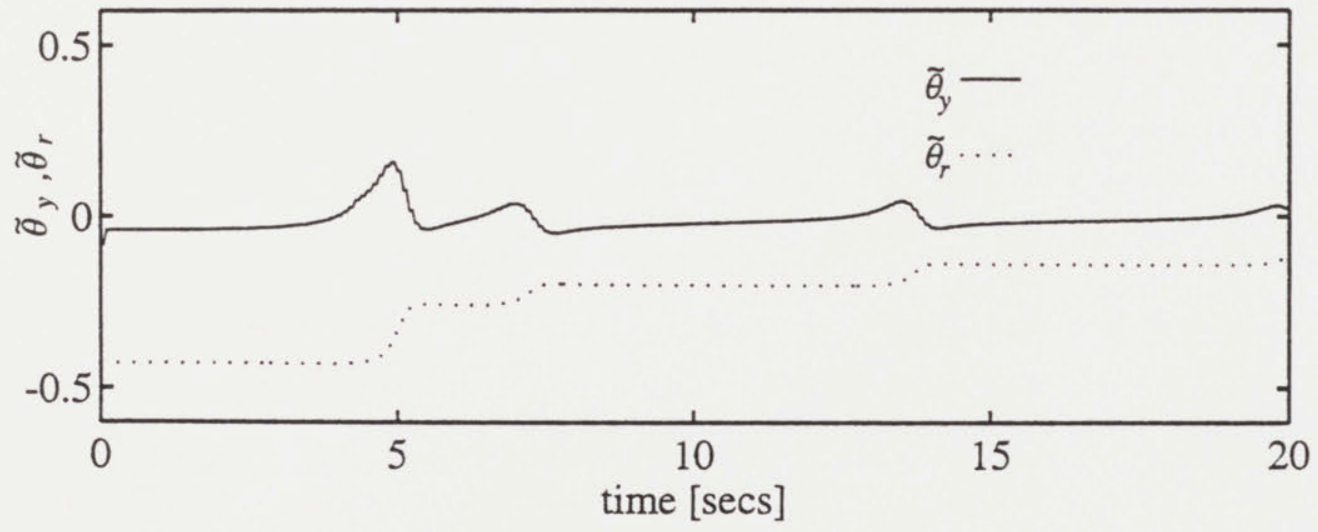

Figure 4-3: Case (c) for Adaptive Control of a 1st-order Discrete-Time Plant. Simulation results for case (c) when the reference input is $r(t)=5 \sin (0.5 t)$. The plots show the time history of the plant and model outputs, the control signal before and after saturation, and parameter errors. 
Plant \& Model Outputs

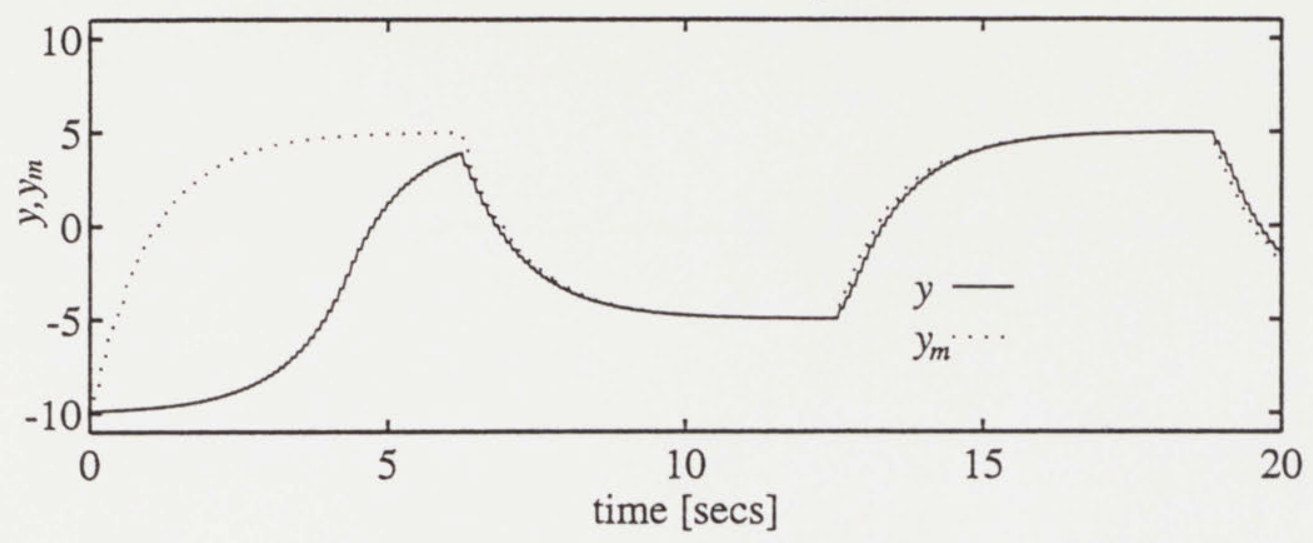

Control Signals

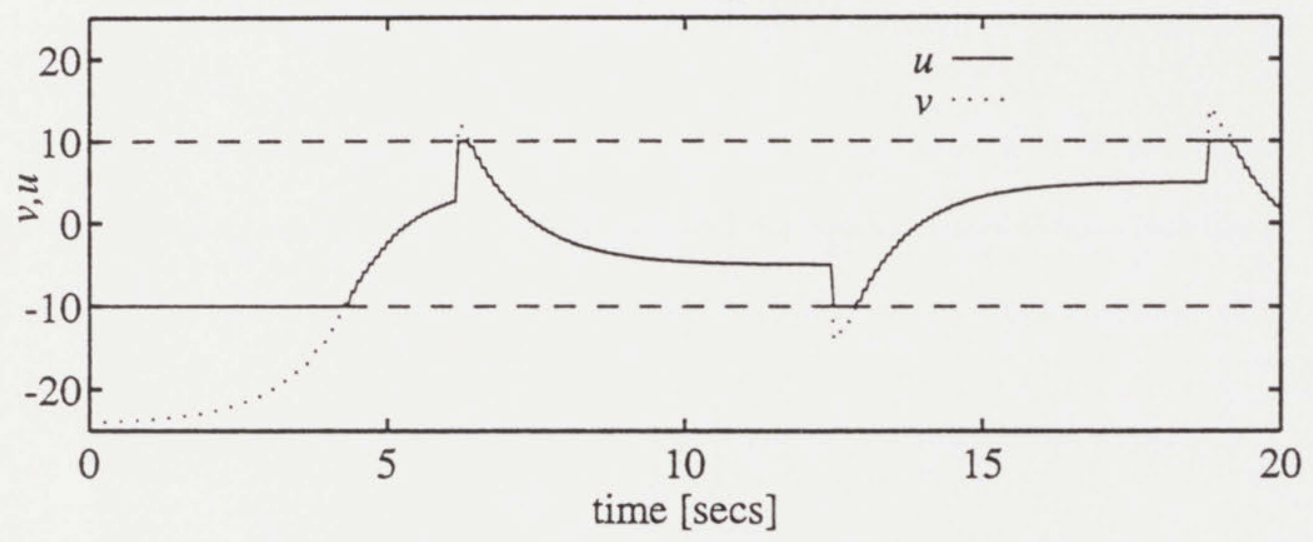

Parameter Errors

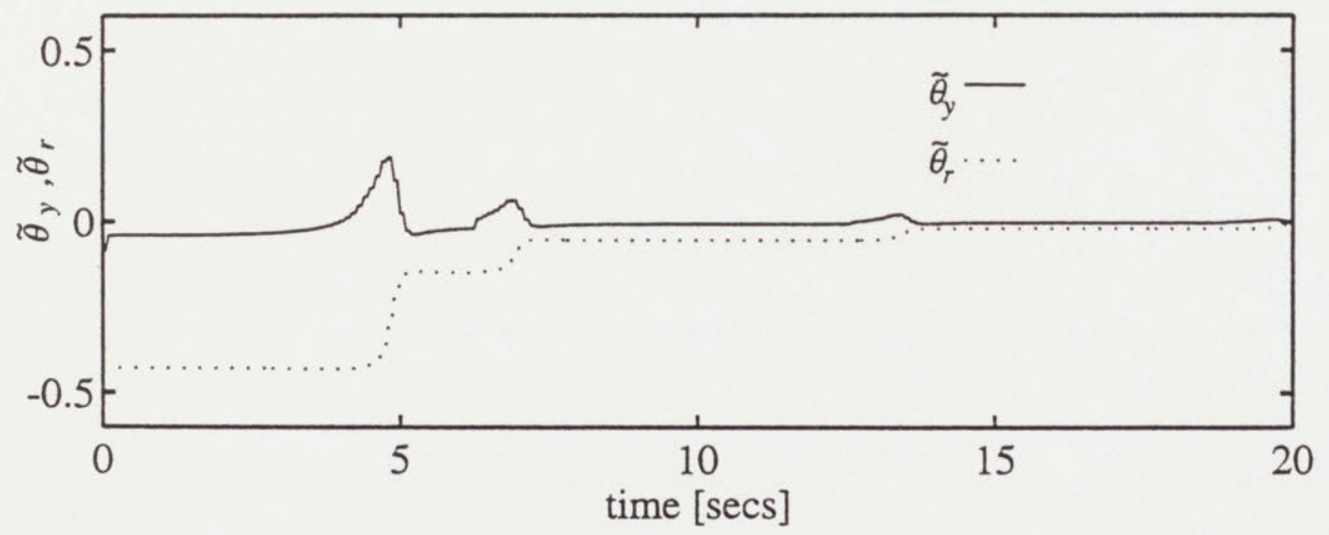

Figure 4-4: Case (d) for Adaptive Control of a 1st-order Discrete-Time Plant. Simulation results for case (d) when the reference input is $r(t)=5 \operatorname{sgn}[\sin (0.5 t)]$. The plots show the time history of the plant and model outputs, the control signal before and after saturation, and parameter errors. 
Plant \& Model Outputs

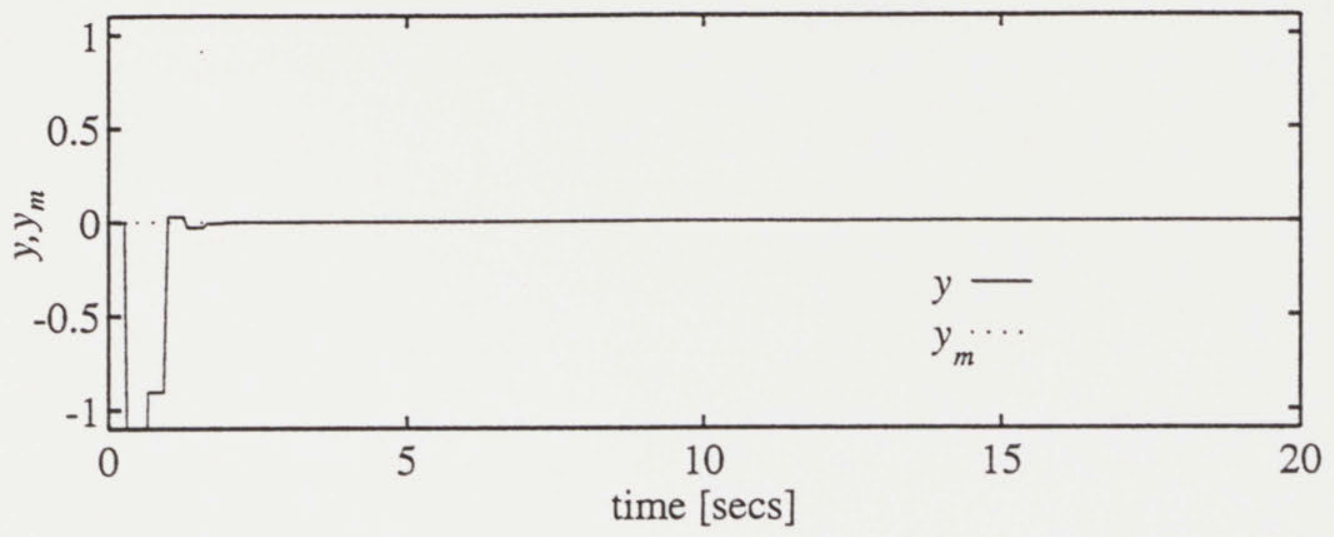

Control Signals

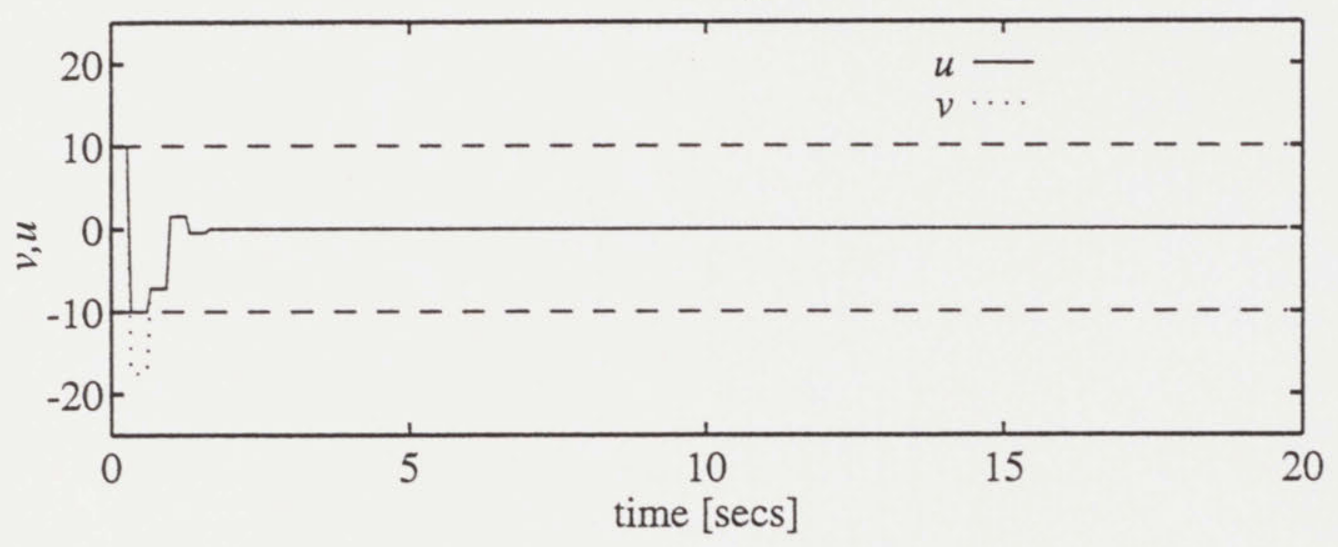

Control Parameter Error

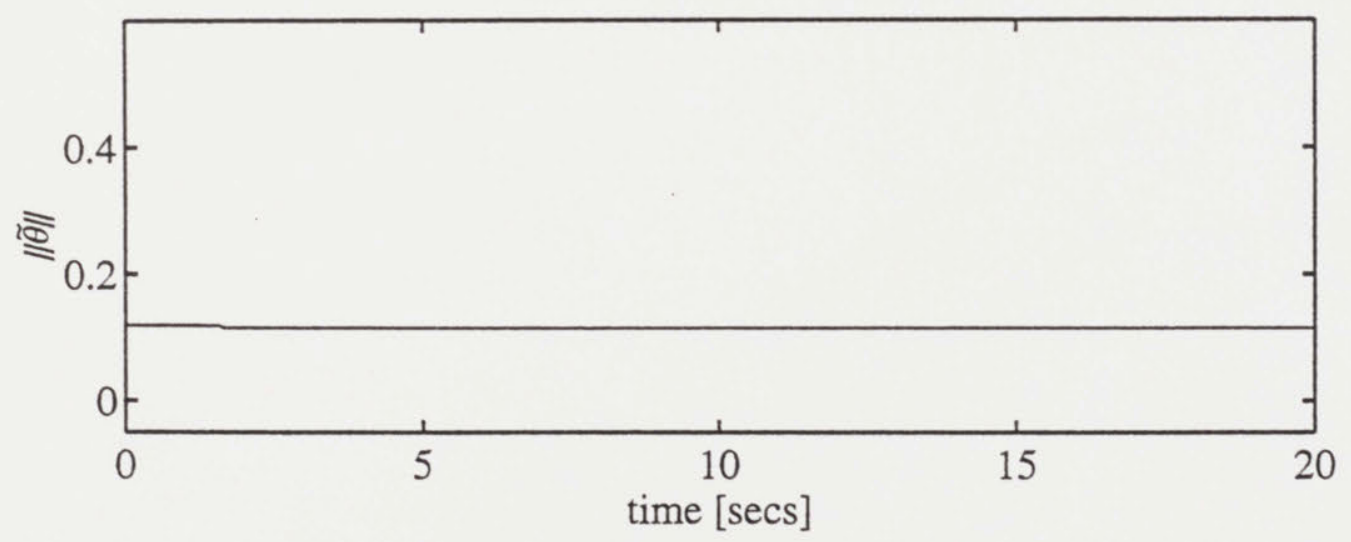

Figure 4-5: Case (a) for One-Step-Ahead Adaptive Control of a Discrete-Time Plant. Simulations for case (a) when the reference output is $y^{*}(t) \equiv 0$. The plots show the time history of the plant and model outputs, the control signal before and after saturation, and the norm of the parameter error vector. 
Plant \& Model Outputs
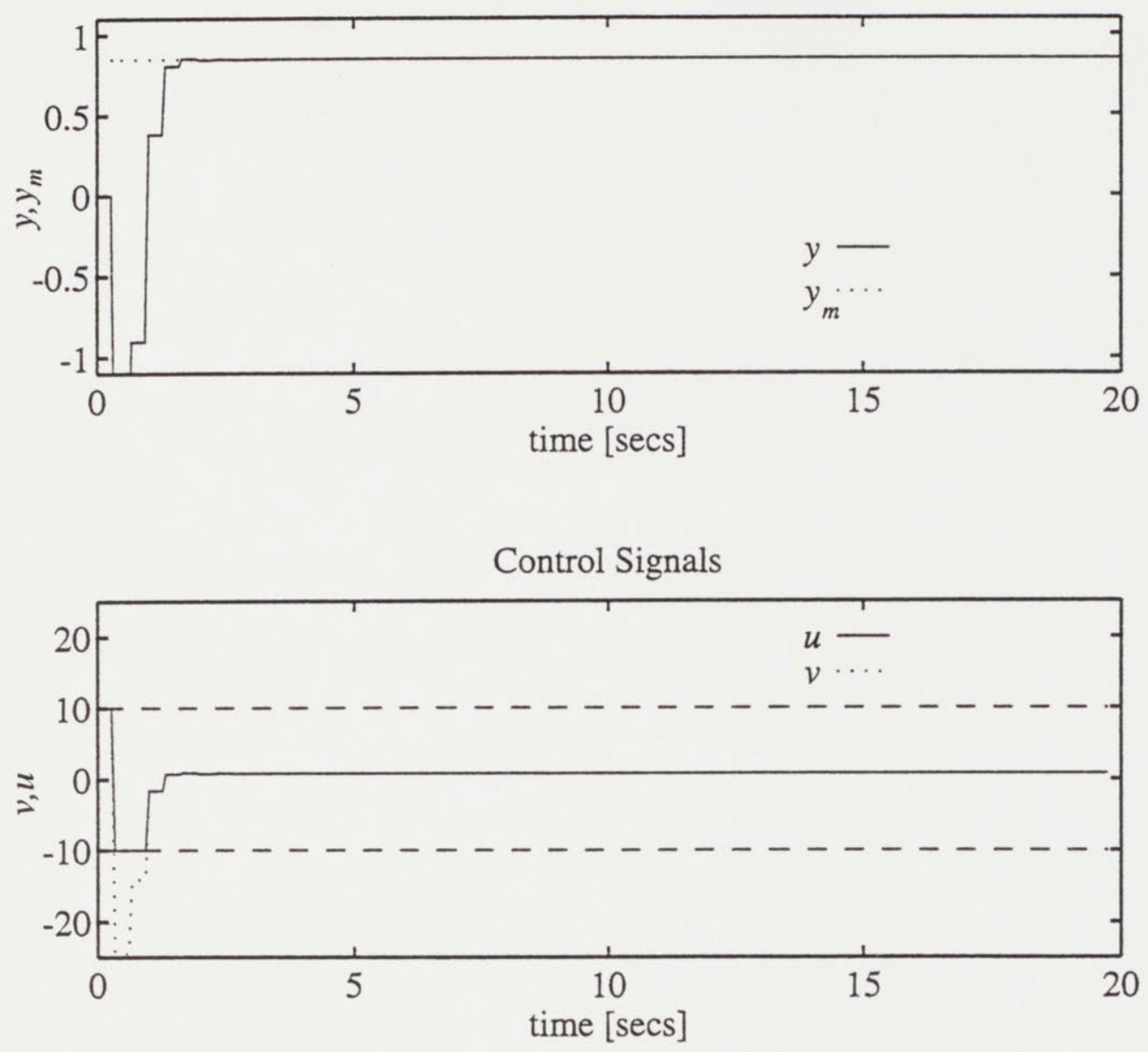

Control Parameter Error

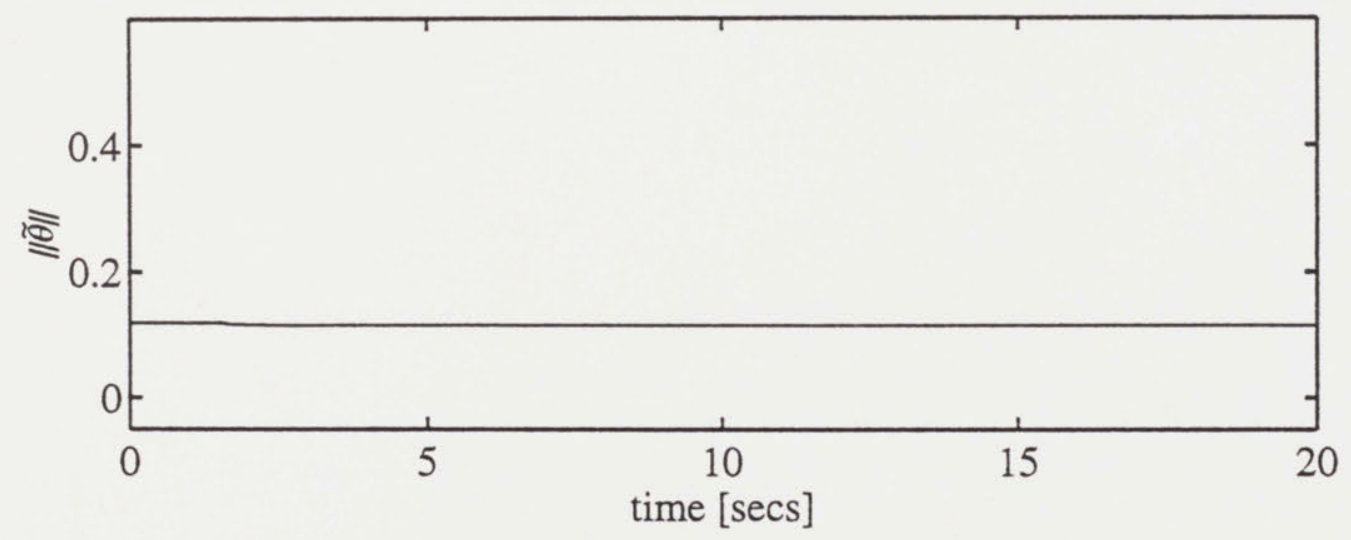

Figure 4-6: Case (b) for One-Step-Ahead Adaptive Control of a Discrete-Time Plant. Simulations for case (b) when the reference output is $y^{*}(t) \equiv 0.8480$. The plots show the time history of the plant and model outputs, the control signal before and after saturation, and the norm of the parameter error vector. 
Plant \& Model Outputs

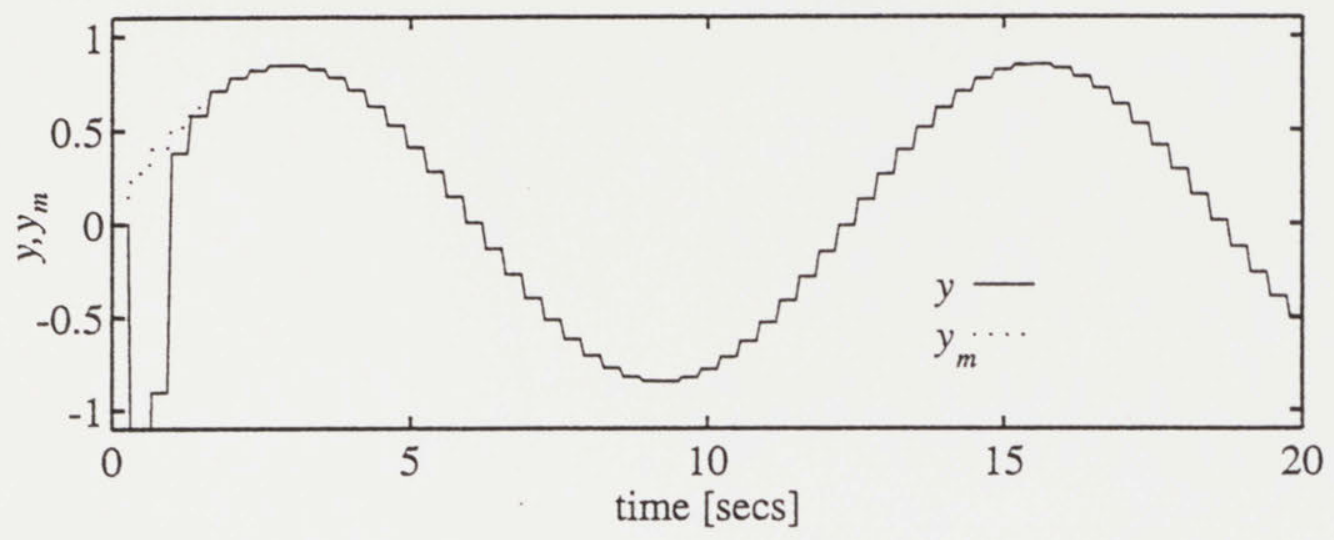

Control Signals

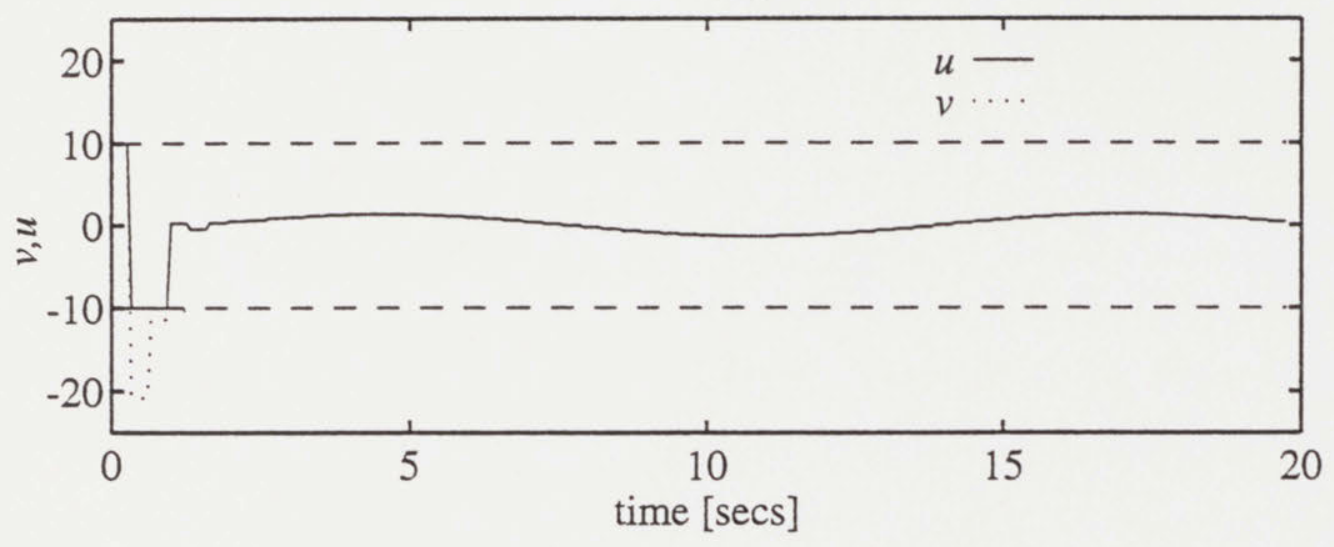

Control Parameter Error

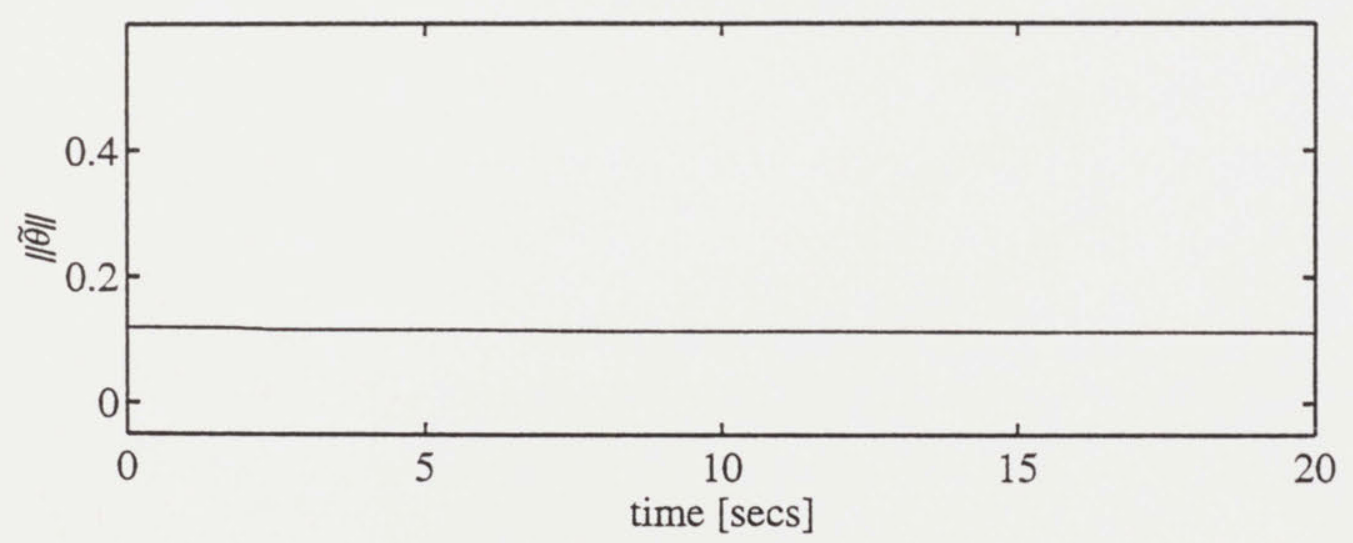

Figure 4-7: Case (c) for One-Step-Ahead Adaptive Control of a Discrete-Time Plant. Simulations for case (c) when the reference output is $y^{*}(t)=0.8480 \sin (0.5 t)$. The plots show the time history of the plant and model outputs, the control signal before and after saturation, and the norm of the parameter error vector. 
Plant \& Model Outputs

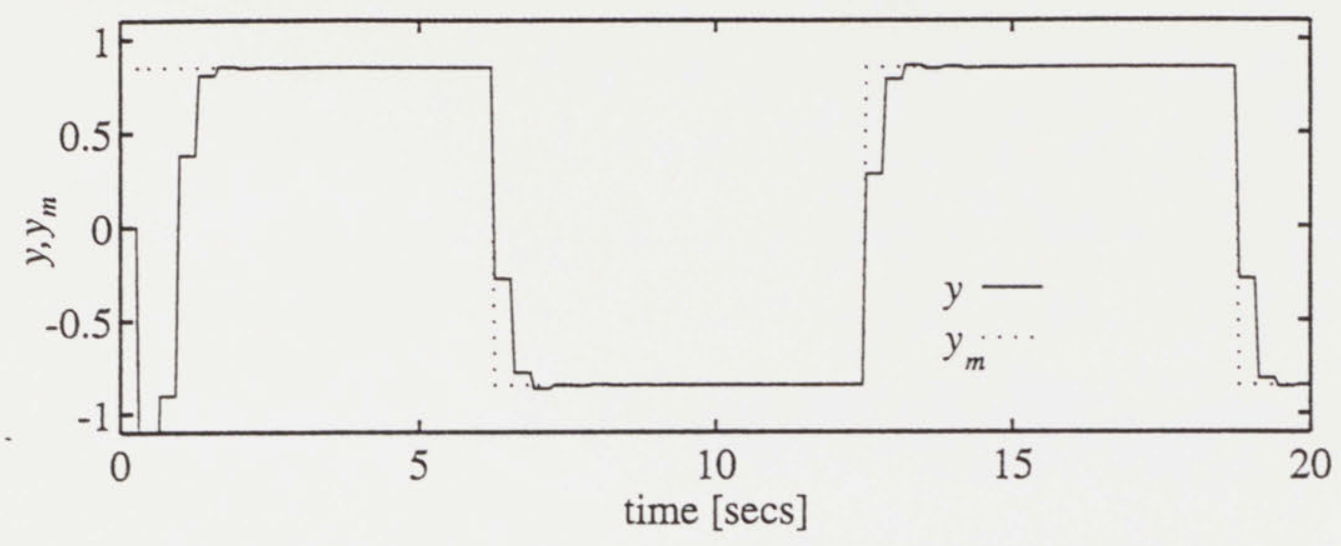

Control Signals

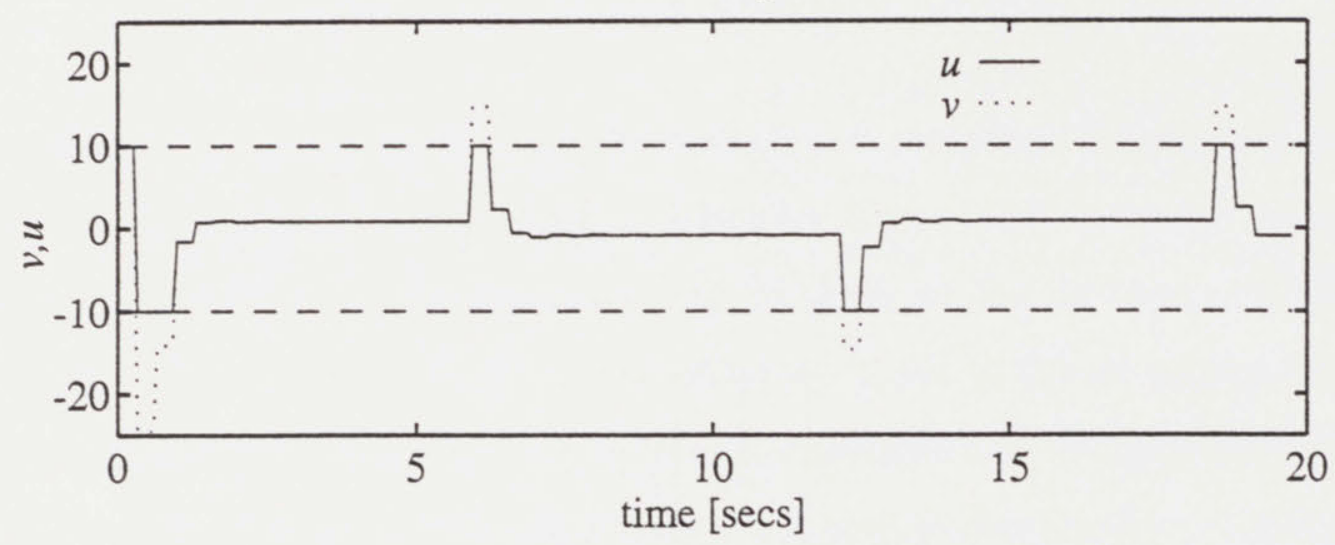

Control Parameter Error

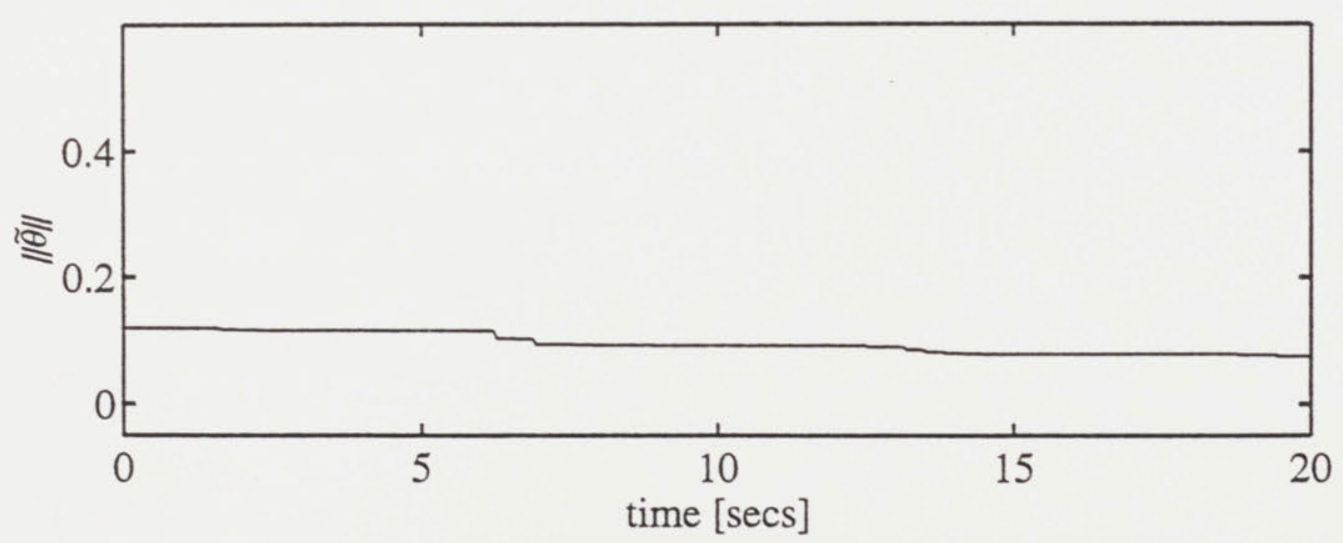

Figure 4-8: Case (d) for One-Step-Ahead Adaptive Control of a Discrete-Time Plant. Simulations for case (d) when the reference output is $y^{*}(t)=0.8480 \operatorname{sgn}[\sin (0.5 t)]$. The plots show the time history of the plant and model outputs, the control signal before and after saturation, and the norm of the parameter error vector. 


\section{Chapter 5}

\section{Application of Bounded Input}

\section{Adaptive Control}

In this chapter it will be shown how the modeling of compliant fingers manipulating a compliant object leads to a problem of adaptive control with bounded input.

In the first section a brief discussion will be given on the problem of object manipulation with human-like hands or end-effectors. Some of the advantages that can be gained from using compliant fingerpads in the end-effectors of multi-multi-purpose robots. Also, an outline is given about how the theory of Haptics describes the touch and manipulation process using models of varying complexity.

The second section is devoted to the analysis of a simplified mathematical model of compliant fingers manipulating a compliant object. Included in the analysis of the model is the identification of the constraints that the manipulation problem imposes on the mathematical model.

Finally the third section deals with the design of an adaptive controller for the dynamic system obtained from the model. It will be shown how this problem naturally leads to amplitude constraints on the control input-force. 


\section{Compliant Fingerpads}

Current robots are in many ways far from achieving a level performance commensurate with that of the human hand. This is especially true for tasks which involve direct manipulation of objects whose characteristics are not necessarily known beforehand.

Robots that are capable of dextrous tasks on a similar level as the human hand are desirable for many reasons. Most obvious are basic industrial applications, such as automated assembly operations. Another very practical application would be the design of robots that are able to perform any kind of critical tasks in a hazardous environment such as high-temperature or high-radiation situations.

One distinguishing characteristic of the human hand is the very sensitive compliant skin-layer on each fingertip. These compliant fingerpads are supported by relatively rigid bones which convey to the skin any forces applied by the muscles attached to the bones. Even though the analysis of the mechanics of the contact is made more complex by allowing the object and the fingers to be compliant, the compliant contact offers several advantages over the rigid contact.

- Compliant fingers contacting a compliant object will generally have a finite area of contact, in contrast to point contact as is the case when the fingers and the object are assumed to be rigid. The introduction of a finite area of contact gives more stable grasp for fewer fingers as then additional resisting and stabilizing torsional torque appears, which resists rotation around the normal to the contact area. The effects of this additional torque can well be seen from the fact that the minimum number of finger contacts to ensure a stable grasp decreases from three to two when the finite are of contact is introduced.

- The stresses resulting in the contact region will be much less in the compliant case as the total force is distributed over the area of contact and not concentrated as in the case of a point contact. This leads to increased dexterity as more fragile objects can then be manipulated.

- Exact shape of the object in the contact region will be less important for stability 
in the compliant case and not as crucial as in the rigid case.

- The explicit modeling of the compliance in the finger and the object will lead to dynamic models of higher order, which to some extent provides increased maneuverability as then the control bandwidth can possibly be increased.

\subsection{Computational Theory of Haptics}

In the computational theory of haptics proposed by Srinivasan and Annaswamy [33], several levels of modeling complexity have been identified and they will now be briefly discussed.

Purely Spatial Sensory is a term used for models which only include the static properties of contact under passive touch. They are mainly useful for the investigation of shape and texture recognition under static situations.

Purely Temporal Sensory-Motor is used to describe models when spatial properties of the contact do not change with deformation during active touch and manipulation. A model of this kind can be realized by assuming that the contact interfaces are rigid, although the sub-surface materials can be compliant. Then the problem can be described using only temporal variables. A model of this type will be analyzed in the next section.

Spatio-Temporal Sensory models describe passive touch when both the spatial an temporal variations contact conditions are is included in the modeling. An example of process a that could be described by a model of this type would be the detection of shape and texture when the object is stroked with a passive hand.

Spatio-Temporal Sensory-Motor are the most general models and include all the factors above. That is, active touch with temporally varying grasping and manipulation, along with spatially and temporally varying contact conditions. 


\section{Dynamic Model}

The general problem of contact between two deformable bodies leads to a spatiotemporal analysis. We simplify the problem by adopting single degree-of-freedom lumped parameter model (See Fig 5-1), thus reducing the variables to be purely temporal. The fingers as well as the object are assumed to deform only in the horizontal direction, defined as $x$. In the $y$ and $z$ directions, with $z$ being positive opposite to the direction of gravity, the fingers and the object behave otherwise as rigid bodies. The aim is to be able to hold a compliant object with unknown dynamic parameters in a two-finger pinch grasp and move it along some desired path in a three-dimensional, gravity environment, while ensuring that the object does not slip or gets crushed.

The dynamic behavior of the two fingers is described by the parameters

$$
\left\{m_{r i}, m_{s i}, b_{c i}, k_{c i}\right\}
$$

where $m_{r i}$ and $m_{s i}$ denote the masses of the rigid backing and the compliant pad, $b_{c i}$ and $k_{c i}$ denote respectively the visco-elastic and elastic coefficients for finger $i$, $i=1,2$. The dynamic properties of the object are also assumed to be described by the parameters

$$
\left\{m_{1}, m_{2}, b, k\right\},
$$

which may be unknown.

The displacements of the rigid backing and the skin of finger $i$ from their equilibrium positions are denoted respectively by the variables $x_{r i}$ and $x_{s i}, i=1,2$. Similarly, $x_{i}$ is the variable describing the displacement of the object mass $m_{i}$ from its equilibrium position.

The variable $f_{x_{i}}$ stands for the external force that is exerted on the rigid backing of finger $i$, and $f_{c i}$ stands for the contact force that results between the fingerpads and the object. The direction of the forces is taken to be positive when they are compressive. This implies that the two fingers are in contact with the object if, $x_{i}=x_{s i}$, and $f_{c i}>0$. When contact is lost, $f_{c i} \equiv 0$ and $x_{s i}$ and $x_{i}$ become independent. 
From now on it will be assumed that the fingers are in contact with the object, then the model for the $x$-direction can be written conveniently as a LTI $8^{\text {th }}$ order 2-input MIMO system in a matrix-vector form:

$$
M \ddot{x}(t)+B \dot{x}+K x=f_{x}
$$

where

$$
\begin{aligned}
& M=\left[\begin{array}{cccc}
m_{r} & 0 & 0 & 0 \\
0 & m_{s}+m_{1} & 0 & 0 \\
0 & 0 & m_{s}+m_{1} & 0 \\
0 & 0 & 0 & m r
\end{array}\right] \quad f_{x}=\left[\begin{array}{c}
f_{x_{1}} \\
0 \\
0 \\
-f_{x_{2}}
\end{array}\right] \quad x=\left[\begin{array}{l}
x_{r_{1}} \\
x_{s_{1}} \\
x_{s_{2}} \\
x_{r_{2}}
\end{array}\right] \\
& B=\left[\begin{array}{cccc}
b_{c_{1}} & -b_{c_{1}} & 0 & 0 \\
-b_{c_{1}} & b+b_{c_{1}} & -b & 0 \\
0 & -b & b+b_{c_{2}} & -b_{c_{2}} \\
0 & 0 & -b_{c_{2}} & b_{c_{2}}
\end{array}\right] \quad K=\left[\begin{array}{cccc}
k_{c_{1}} & -k_{c_{1}} & 0 & 0 \\
-k_{c_{1}} & k+k_{c_{1}} & -k & 0 \\
0 & -k & k+k_{c_{2}} & -k_{c_{2}} \\
0 & 0 & -k_{c_{2}} & k_{c_{2}}
\end{array}\right]
\end{aligned}
$$

By introducing some symmetries into the problem the control task can be simplified further. First it is assumed that the left and right finger are identical in their dynamic characteristics so that $m_{r 1}=m_{r 2} \triangleq m_{r}, m_{s 1}=m_{s 2} \triangleq m_{s}, b_{c_{1}}=b_{c_{2}} \triangleq b_{c}$, and $k_{c_{1}}=k_{c_{2}} \triangleq k_{c}$. Secondly it is assumed that the object is symmetric about its center so that $m_{1}=m_{2}$. It is shown in [34] that with these simplifications this MIMO system can be transformed into two decoupled SISO systems by using state transformations. The state-transformation is realized by defining an asymmetric part and a 
symmetric part for each variable can as follows

$$
\begin{array}{rr}
x_{r_{1}}(t)=x_{r a}(t)+x_{r s}(t) & x_{r_{2}}(t)=x_{r a}(t)-x_{r s}(t) \\
x_{s_{1}}(t)=x_{s a}(t)+x_{s s}(t) & x_{s_{2}}(t)=x_{s a}(t)-x_{s s}(t) \\
\left.f_{x_{1}}\right)(t)=f_{x s}(t)+f_{x a}(t) & f_{x_{2}}(t)=f_{x s}(t)-f_{x a}(t) \\
\left.f_{c x_{1}}\right)(t)=f_{c x s}(t)+f_{c x a}(t) & f_{c x_{2}}(t)=f_{c x s}(t)-f_{c x a}(t) .
\end{array}
$$

Now the system can be written using this state-space representation as two decoupled systems

$$
\dot{x}_{a}=A_{a} x_{a}+b_{a} f_{x a} \quad \dot{x}_{s}=A_{s} x_{s}+b_{s} f_{x s}
$$

where

$$
\begin{aligned}
& A_{a}=\left[\begin{array}{cccc}
0 & 1 & 0 & 0 \\
\frac{-k_{c}}{m_{s}+m_{1}} & \frac{-b_{c}}{m_{s}+m_{1}} & \frac{k_{c}}{m_{s}+m_{1}} & \frac{b_{c}}{m_{s}+m_{1}} \\
0 & 0 & 0 & 1 \\
\frac{k_{c}}{m_{r}} & \frac{b_{c}}{m_{r}} & \frac{-k_{c}}{m_{r}} & \frac{-b_{c}}{m_{r}}
\end{array}\right] b_{a}=\left[\begin{array}{c}
0 \\
0 \\
0 \\
\frac{1}{m_{r}}
\end{array}\right] x_{a}=\left[\begin{array}{c}
x_{s a} \\
\dot{x}_{s a} \\
x_{r a} \\
\dot{x}_{r a}
\end{array}\right] \\
& A_{s}=\left[\begin{array}{cccc}
0 & 1 & 0 & 0 \\
-\frac{k_{c}+2 k}{m_{s}+m_{1}} & -\frac{b_{c}+2 b}{m_{s}+m_{1}} & \frac{k_{c}}{m_{s}+m_{1}} & \frac{b_{c}}{m_{s}+m_{1}} \\
0 & 0 & 0 & 1 \\
\frac{k_{c}}{m_{r}} & \frac{b_{c}}{m_{r}} & \frac{-k_{c}}{m_{r}} & \frac{-b_{c}}{m_{r}}
\end{array}\right] b_{s}=\left[\begin{array}{c}
0 \\
0 \\
0 \\
\frac{1}{m_{r}}
\end{array}\right] x_{a}=\left[\begin{array}{c}
x_{s s} \\
\dot{x}_{s s} \\
x_{r s} \\
\dot{x}_{r s}
\end{array}\right]
\end{aligned}
$$

This model can be used to describe the $x$-dimensional dynamics of the object. In the other directions ( $y$ and $z$ ) it is assumed for simplicity that the system behaves as 
a rigid body in these directions, or

$$
\begin{aligned}
& m_{t} \ddot{y}(t)=f_{y_{1}}(t)+f_{y_{2}}(t) \\
& m_{o} \ddot{y}(t)=f_{c y_{1}}(t)+f_{c y_{2}}(t) \\
& m_{t} \ddot{z}(t)=-m_{t} g+f_{z_{1}}(t)+f_{z_{2}}(t) \\
& m_{o} \ddot{y}(t)=-m_{o} t+f_{c z_{1}}(t)+f_{c z_{2}}(t)
\end{aligned}
$$

where $m_{t} \triangleq 2 m_{\tau}+2 m_{s}+2 m_{1}$ and $m_{o} \triangleq 2 m_{1}$.

\subsection{Constraints}

When writing Eq. (5.1) it was assumed that the contact force was positive, but it must also be ensured that the normal contact force is sufficiently large to prevent slip in either $y$ or $z$ direction. Assuming that Coloumb's friction law can be applied at the contact surface then this constraint becomes

$$
f_{c x_{i}}(t)>\frac{1}{\mu} \sqrt{f_{c z_{i}}(t)^{2}+f_{c y_{i}}(t)^{2}} \quad \forall t \geq t_{0} \quad i=1,2
$$

where $\mu$ is the coefficient of friction. Further, assuming that $f_{y_{1}} \equiv f_{y_{2}}$ and $f_{z_{1}} \equiv f_{z_{2}}$ then the constraint can be expressed using the alternative state variables as

$$
\left|f_{x c a}(t)\right|+\frac{1}{\mu} \sqrt{f_{c z_{i}}(t)^{2}+f_{c y_{i}}(t)^{2}}<f_{x c s}(t) \quad \forall t \geq t_{0}
$$

A second constraint can also be imposed by assuming that the object can only tolerate a finite amount of compression force, $f_{\text {crush }}$, before being crushed. This can be formulated as

$$
f_{x c s}(t) \leq f_{\text {crush }}
$$

\subsection{Satisfaction of Constraints}

Any control scheme considered must ensure that the constraints in Eqs. (5.4) and (5.5) are satisfied at all times. That problem can be reduced to constraining the external forces $f_{a}, f_{s}, f_{y}$ and $f_{z}$ to lie within a compact set, as will now be shown. 
It can shown that the external forces are related to the contact forces through transfer functions as follows

$$
\begin{aligned}
\frac{f_{c a}(s)}{f_{a}(s)} & =\frac{m_{1} b_{c} s+m_{1} k_{c}}{\bar{m} s^{2}+m_{t} b_{c} s+m_{t} b_{c}} \\
\frac{f_{c s}(s)}{f_{s}(s)} & =\frac{m_{1} b_{c} s^{3}+\left(m_{1} k_{c}+2 b b_{c}\right) s^{2}+2\left(b k_{c}+b_{c} k\right) s+2 k k_{c}}{\bar{m} s^{4}+\left(2 m_{r} b+m_{t} b_{c}\right) s^{3}+\left(2 m_{r} b+m_{t} k_{c}+2 b b_{c}\right) s^{2}+2\left(b k_{c}+b_{c} k\right) s+2 k k_{c}} \\
\frac{f_{c y}(s)}{f_{y}(s)} & =\frac{m_{1}}{m_{t}} \\
\frac{f_{c z}(s)}{f_{z}(s)} & =\frac{m_{1}}{m_{t}}
\end{aligned}
$$

where $\bar{m} \triangleq m_{r}\left(m_{s}+m_{1}\right)$. It is noted that all these transfer functions are stable and proper and therefore have finite gain for all frequencies. This means that the gains of the transfer functions give bounds on the time-signals as well or

$$
\begin{aligned}
& \left|f_{c a}(t)\right| \leq \gamma_{a}^{\max } \forall t \geq t_{0} \\
& \left|f_{c y}(t)\right| \leq \gamma_{b}^{\max }\left|f_{y}(t)\right| \forall t \geq t_{0} \\
& \left|f_{c z}(t)\right| \leq \gamma_{b}^{\max }\left|f_{z}(t)\right| \forall t \geq t_{0}
\end{aligned}
$$

where $\gamma_{a}^{\max }, \gamma_{y}^{\max }$ and $\gamma_{z}^{\max }$ are determined from the corresponding transfer function in Eq. (5.6). The signal, $f_{s}$ needs to be generated in a such way that it contains a governing zero-frequency, (dc) component that is of sufficient magnitude to guarantee that

$$
\gamma_{s}^{\min }\left|f_{s}(t)\right| \leq\left|f_{c s}(t)\right| \leq \gamma_{s}^{\max } \forall t \geq t_{0}
$$

where $\gamma_{s}^{\min }$ and $\gamma_{s}^{\max }$ are determined from the transfer function in (5.6). Using the above in Eq. (5.4) and (5.5) it is obtained that the constraints are satisfied as long as

$$
\gamma_{a}^{\max }\left|f_{a}(t)\right|+\frac{\gamma_{b}^{\max }}{\mu} \sqrt{f_{y}^{2}(t)+f_{z}^{2}(t)} \leq \gamma_{s}^{\min } f_{s}(t)
$$


and that the object does not get crushed if

$$
\gamma_{s}^{\max }\left|f_{s}(t)\right| \leq f_{\text {crush }}
$$

. Given bounds, $F_{s}^{\min }$, and $F_{b}^{\max }$ such that $\sqrt{f_{y}^{2}(t)+f_{z}^{2}(t)} \leq F_{b}^{\max } \forall t \geq t_{0}$, and $f_{s}(t) \geq F_{s}^{\min } \forall t \geq t_{0}$, then the final result is that the object does not slip if

$$
\left|f_{a}(t)\right| \leq \frac{1}{\gamma_{a}^{\max }}\left[\gamma_{s}^{\min } F_{s}^{\min }-\frac{\gamma_{b}^{\max } F_{b}^{\max }}{\mu}\right] \triangleq f_{a_{0}}
$$

The problem of preventing slip has now been reduced to a control problem in presence of amplitude constraints.

\section{Control Design}

Often, as in the case of a general purpose robot, the objects encountered can possess a wide range of dynamic characteristics. This implies that the grasping and manipulation tasks have to be performed when the dynamics of the object in question is unknown. In such cases, the control forces on the fingerpads need to be generated using an adaptive rather than a fixed controller.

\subsection{An Adaptive Controller}

The manipulation task is to generate forces $f_{x_{i}}$ so that the object position $x_{s a}$ follows a desired path $x_{s a}^{*}(t)$ for all time $t$, and since the object has unknown characteristics, an adaptive controller is required to control the system in Eq. (5.2). As mentioned in section 2.2, the magnitude constraint on $\left|f_{c a}\right|$ is satisfied if $\left|f_{a}(t)\right| \leq f_{a_{0}}$ as determined in Eq. (5.8). Hence the problem is to determine an adaptive controller to generate the input $f_{a}$ in Eq. (5.2) in the presence of the magnitude constraint

$$
\left|f_{a}(t)\right| \leq f_{a_{0}} \quad \forall t \geq t_{0} .
$$


An input-output relation can be determined from Eq. (5.2) as

$$
\frac{x_{s a}(s)}{f_{x a}(s)}=\frac{b_{c} s+k_{c}}{s^{2}\left[m_{r}\left(m_{1}+m_{s}\right) s^{2}+\left(m_{r}+m_{s}+m_{1}\right)\left(b_{c} s+k_{c}\right)\right]} .
$$

It is seen that this transfer function has relative degree $n^{*}=3$ and hence the results of chapter 3 section 3 can be used when designing a controller. A fourth-order reference model is chosen with a transfer function $W_{m}(s)$ so that it represents the best possible prior information about the object parameters. A reference input $f_{a r}$ is selected so that the corresponding output is the desired path motion $x_{s a}^{*}$ and we let $\left|f_{a r}\right| \leq f_{a r_{0}}$. The adaptive controller is described by the equations

$$
\begin{aligned}
& \dot{\omega}_{1}(t)=\Lambda \omega_{1}(t)+l f_{a}(t) \\
& \dot{\omega}_{2}(t)=\Lambda \omega_{2}(t)+l x_{s a}(t) \\
& \omega(t)=\left[f_{a r}(t), \omega_{1}(t)^{T}, x_{s a}(t), \omega_{2}(t)^{T}\right] \\
& \theta(t)=\left[k(t), \theta_{1}(t)^{T}, \theta_{0}(t), \theta_{2}(t)^{T}\right] \\
& \bar{f}_{a}(t)=\theta(t)^{T} \omega(t) \\
& f_{a}(t)=\left\{\begin{array}{lll}
\bar{f}_{a}(t) & \text { if } & \left|\bar{f}_{a}(t)\right| \leq f_{a_{0}} \\
f_{a_{0}} \operatorname{sgn}\left(\bar{f}_{a}(t)\right) & \text { if } & \left|\bar{f}_{a}(t)\right|>f_{a_{0}}
\end{array}\right. \\
& e_{\Delta}(t)=k_{\Delta}(t) W_{m}(s)\left(f_{a}(t)-\bar{f}_{a}(t)\right) \\
& e_{u 1}(t)=x_{s a}(t)-x_{s a}^{*}(t)-e_{\Delta}(t) \\
& e_{2}(t)=\left[\theta(t)^{T} W_{m}(s) I-W_{m}(s) \theta^{T}(t)\right] \omega(t) \\
& \varepsilon_{1 u}(t)=e_{u 1}(t)+k_{1}(t) e_{2}(t)
\end{aligned}
$$




$$
\begin{aligned}
\dot{\theta}(t) & =\dot{\phi}(t)=-\operatorname{sgn}\left(k_{p}\right) \frac{\varepsilon_{1 u}(t) \xi(t)}{1+\xi^{T}(t) \xi(t)} \\
\dot{k}_{1}(t) & =\dot{\psi}_{1}(t)=-\frac{\varepsilon_{1 u}(t) e_{2}(t)}{1+\xi^{T}(t) \xi(t)} \\
\dot{k}_{\Delta}(t) & =-\dot{\psi}_{\Delta}(t)=\frac{\varepsilon_{1 u}(t) \xi_{\Delta}(t)}{1+\xi^{T}(t) \xi(t)}
\end{aligned}
$$

where $k: \mathbb{R}^{+} \rightarrow \mathbb{R}, \theta_{1}, \omega_{1}: \mathbb{R}^{+} \rightarrow \mathbb{R}^{3}, \theta_{0}: \mathbb{R}^{+} \rightarrow \mathbb{R}, \theta_{2}, \omega_{2}: \mathbb{R}^{+} \rightarrow \mathbb{R}^{3}$, $\Lambda \in \mathbb{R}^{3 \times 3}$ is an asymptotically stable matrix, also $\xi(t)=W_{m}(s) I \omega(t), \xi_{\Delta}(t)=$ $W_{m}(s)\left(f_{a}(t)-\bar{f}_{a}(t)\right)$.

Remark: The controller given above is high-order and quite complicated and needs further simplification before it can be considered practical. It is noted however that it is the structure of the system and the controller that is the reason for this complexity and not the requirement that the control input be bounded in magnitude. 


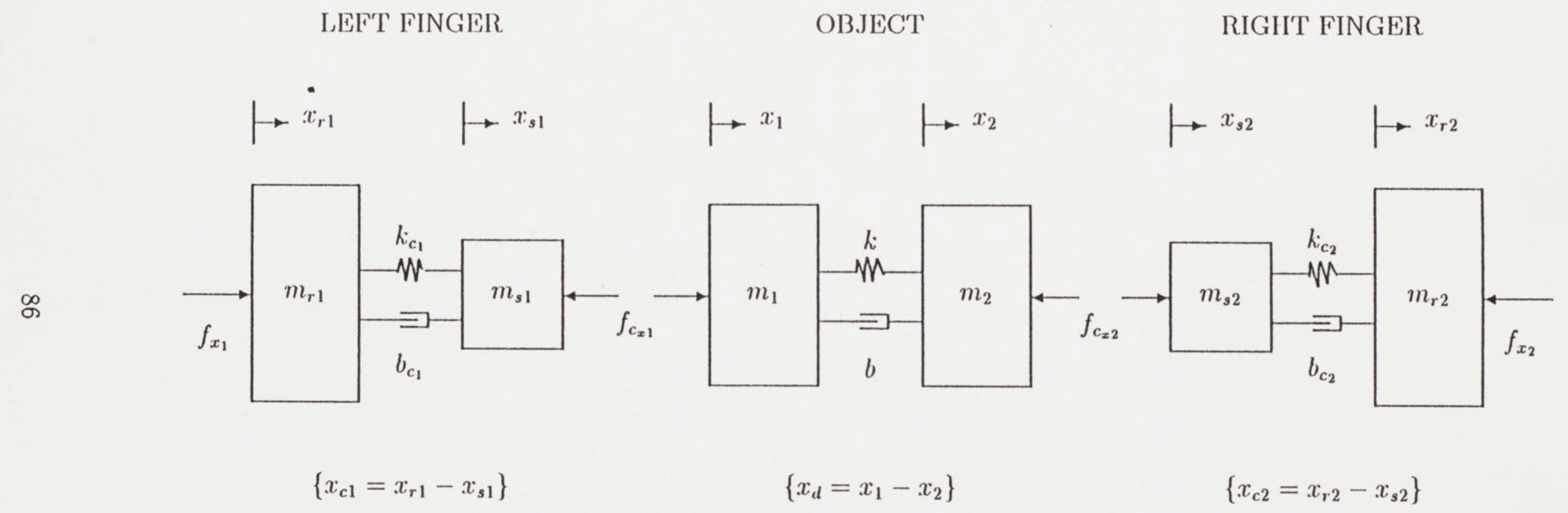

Figure 5-1: A schematic diagram of the dynamic model. 


\section{Chapter 6}

\section{Summary and Conclusions}

\section{Summary}

In this thesis the problem of adaptively controlling a linear time-invariant plant in the presence of constraints on the input amplitude has been solved to some extent. New algoritms were introduced for adaptive control of a continuous-time plants and it shown that they lead to bounded solutions when the initial conditions of the adaptive system are within a compact set. A similar condition was also put forward for discretetime plants which similarily ensured bounded trajectories. In both cases the results are valid for open-loop not necessarily stable plants but minimum phase. It was also shown how the control problem involved in the manipulation of a compliant object with compliant fingerpads, quite naturally reduces to a problem of adaptive control in the presence of input constraints. A controller based on the previously obtained results and which solved that problem was given.

\section{Conclusions}

In this thesis some light has been cast on how saturation effects the stability of adaptive systems. Many questions are however, still unanswered on the various interesting phenomena that can evolve in a non-linear and time-varying system as the one in question. For example, it would be interesting to know under what conditions, a limit 
cycle can appear. Also a interesting question is to ask which initial conditions and reference inputs lead to control signals that are high enough in amplitude to cause saturation. These questions and many more are the subject of future research. 


\section{Appendix A}

\section{Proofs of Theorems in Chapter 3}

\section{Proof of Theorem 3.1}

The proof is given by showing the existence of a compact set $\mathcal{A}$ in the state space which bounds the trajectories.

The following definitions are useful to prove Theorem 3.1.

$$
\begin{aligned}
W\left(x_{p}\right) & \triangleq x_{p}^{2} / 2 \\
f\left(x_{p}\right) & \triangleq a_{m} x_{p}^{2}+b_{p} \phi x_{p}^{2}+b_{p} \psi r+k^{*} b_{p} r x_{p} \\
\mathcal{A} & \triangleq\left\{x_{p}\left|\frac{\left|b_{p}\right| r_{0}\left(\alpha \phi_{\max }+\left|k^{*}\right|\right)}{\left|a_{m}\right|-\left|b_{p}\right| \phi_{\max }} \leq\right| x_{p}|\leq| \frac{u_{0} b_{p}}{a_{p}} \mid\right\} .
\end{aligned}
$$

Condition (ii) in Theorem 3.1 implies that

$$
\phi_{\max } \leq \frac{\left|a_{m}\right|-\left|k^{*}\right| \frac{r_{0}}{u_{0}}\left|a_{p}\right|}{\left|b_{p}\right|+\alpha \frac{r_{0}}{u_{0}}\left|a_{p}\right|} .
$$

Also from the definition of $\mathcal{A}$ and $\phi_{\max }$, it follows that

$$
f\left(x_{p}\right) \leq a_{m}\left|x_{p}\right|\left\{\left(1-\left|\frac{b_{p}}{a_{m}}\right| \phi_{\max }\right)\left|x_{p}\right|-\left|\frac{b_{p} r_{0}}{a_{m}}\right|\left(\alpha \phi_{\max }+\left|k^{*}\right|\right)\right\} \leq 0 \quad \forall x_{p} \in \mathcal{A} .
$$

The proof is provided by considering the cases when (a) $|v| \leq u_{0}$ and (b) $|v|>u_{0}$. 
(a) Let $|v| \leq u_{0}$. Then

$$
u(t)=v(t)=\theta(t) x_{p}(t)+k(t) r(t)
$$

and $\Delta u(t)=0$. Hence

$$
\dot{x}_{p}(t)=a_{m} x_{p}(t)+b_{p} \phi(t) x_{p}(t)+b_{p} \psi(t) r(t)+b_{p} k^{*} r(t) .
$$

This leads to the time derivative

$$
\begin{aligned}
\dot{W}\left(x_{p}\right) & =a_{m} x_{p}^{2}+b_{p} \phi x_{p}^{2}+b_{p} \psi r+k^{*} b_{p} r x_{p} \\
& \leq 0 \quad \forall x_{p} \in \mathcal{A} \quad \text { from Eq. (A.2). }
\end{aligned}
$$

(b) Let $|v|>u_{0}$. Here, $u=u_{0} \operatorname{sgn}(v)$ and $|v|>u_{0}$. As a result, Eq. (3.4) becomes

$$
\dot{x}_{p}(t)=a_{m} x_{p}(t)+b_{p} u_{0} \operatorname{sgn}(v(t))-b_{p} \theta^{*} x_{p}(t)
$$

and correspondingly,

$$
\begin{aligned}
\dot{W}\left(x_{p}\right) & =\left[a_{m} x_{p}^{2}-b_{p} \theta^{*} x_{p}^{2}+b_{p} u_{0} \operatorname{sgn}(v) x_{p}\right] \\
& =\left[a_{m} x_{p}^{2}-b_{p} \theta^{*} x_{p}^{2}+u_{0}\left|b_{p} x_{p}\right| \operatorname{sgn}(v) \operatorname{sgn}\left(b_{p} x_{p}\right)\right] .
\end{aligned}
$$

It will be shown that $\dot{W}\left(x_{p}\right)<0 \forall x_{p} \in \mathcal{A}$ by considering two separate cases, based on the signs of $v$ and $b_{p} x_{p}$.

$\underline{\text { Case I } \operatorname{sgn}(v)=\operatorname{sgn}\left(b_{p} x_{p}\right)}$. Since $|v|>u_{0}, v b_{p} x_{p} \geq u_{0}\left|b_{p} x_{p}\right|$. Hence

$$
b_{p} \theta^{*} x_{p}^{2}+b_{p} \phi x_{p}^{2}+b_{p} k r x_{p} \geq u_{0}\left|b_{p} x_{p}\right|
$$

or

$$
-\left|a_{m}\right| x_{p}^{2}-b_{p} \theta^{*} x_{p}^{2}+\left|b_{p} x_{p}\right| u_{0}+\left[\left|a_{m}\right| x_{p}^{2}-b_{p} \phi x_{p}^{2}-b_{p} \psi x_{p} r-b_{p} k^{*} r x_{p}\right]<0 .
$$


It is also noted that

$$
\dot{W}\left(x_{p}\right)=-\left|a_{m}\right| x_{p}^{2}-b_{p} \theta^{*} x_{p}^{2}+u_{0}\left|b_{p} x_{p}\right|=a_{p} x_{p}^{2}+u_{0}\left|b_{p} x_{p}\right| .
$$

If $\dot{W}\left(x_{p}\right)>0$, it follows from Eq. (A.4) that the bracketed term is negative. This contradicts Eq. (A.2) which implies that Case I cannot occur for any $x_{p} \in \mathcal{A}$.

Case II $\operatorname{sgn}(v)=-\operatorname{sgn}\left(b_{p} x_{p}\right)$.

Here $\dot{W}$ reduces to

$$
\dot{W}\left(x_{p}\right)=a_{p} x_{p}^{2}-\left|b_{p} x_{p} u_{0}\right|
$$

Since $\left|x_{p}\right| \leq\left|\frac{b_{p} u_{0}}{a_{p}}\right|$ in $\mathcal{A}$, once again $\dot{W}\left(x_{p}\right) \leq 0 \forall x_{p} \in \mathcal{A}$ in case II.

It is noted that $\mathcal{A}$ is non-empty since

$$
\phi_{\max } \leq \frac{\left|a_{m}\right|-k^{*} \frac{r_{0}}{u_{0}}\left|a_{p}\right|}{\left|b_{p}\right|+\alpha \frac{r_{0}}{u_{0}}\left|a_{p}\right|} \quad \Rightarrow \quad \frac{\left|b_{p}\right| r_{0}\left(\alpha \phi_{\max }+\left|k^{*}\right|\right)}{\left|a_{m}\right|-\left|b_{p}\right| \phi_{\max }} \leq\left|\frac{u_{0} b_{p}}{a_{p}}\right|
$$

which concludes the proof.

\section{Proof of Theorem 3.2}

Proof: Define $W\left(x_{p}\right)=x_{p}^{T} P x_{p}$ and

$$
\mathcal{A} \triangleq\left\{x_{p} \mid \frac{2 p_{b} r_{0}}{q_{0}-\phi_{\max }\left[2 p_{b}+\alpha\left|k^{*}\right|\left(q_{0}+2 p_{b}\left\|\theta^{*}\right\|\right)\right]}<\left\|x_{p}\right\|<\frac{2 p_{b} u_{0}}{\left|k^{*}\right|\left|2 p_{b}\left\|\theta^{*}\right\|-q_{0}\right|}\right\} .
$$

Condition (ii) implies that

$$
\phi_{\max }<\frac{q_{0}-\rho\left|k^{*}\right| \frac{r_{0}}{u_{0}}\left|2 p_{b}\left\|\theta^{*}\right\|-q_{0}\right|}{2 p_{b}+\alpha\left|k^{*}\right|\left[q_{0}+2 p_{b}\left\|\theta^{*}\right\|\right]} .
$$

As in the proof of Theorem 3.1, two cases are considered.

(a) Let $|v| \leq u_{0}$. Here $\Delta u=0$, and hence

$$
u(t)=v(t)=k(t) \theta^{T}(t) x_{p}(t)+k(t) r(t) .
$$


As a result,

$$
\dot{x}_{p}(t)=A_{m} x_{p}+b_{m}[\phi(t)+\psi(t) k(t) \theta(t)]^{T} x_{p}(t)+b_{p} k(t) r(t) .
$$

This leads to a time-derivative

$$
\begin{aligned}
\dot{W}\left(x_{p}\right) & =x_{p}^{T}\left[A_{m}^{T} P+P A_{m}^{T}\right] x_{p}+2\left(b_{m}^{T} P x_{p}\right)[\phi+\psi k \theta]^{T} x_{p}+2\left(b_{m}^{T} P x_{p}\right) \frac{k}{\left|k^{*}\right|} r \\
& =-q_{0}\left\|x_{p}\right\|^{2}+2 p_{b}\left\|x_{p}\right\|^{2} \frac{\phi_{\max }+\left|k^{*}\right| \alpha \phi_{\max } \theta^{*}}{1-\left|k^{*}\right| \alpha \phi_{\max }}+2 p_{b}\left\|x_{p}\right\| \frac{r_{0}}{1-\left|k^{*}\right| \alpha \phi_{\max }} \\
& < \\
0 & \forall x_{p} \in \mathcal{A}
\end{aligned}
$$

which follows from the definition of $\mathcal{A}$ and the condition in Theorem 3.2 and where it has been used that $|\psi| \leq \alpha \phi_{\max }$ and therefore

$$
\sup _{t}(|k(t) \psi(t)|) \leq \frac{\left|k^{*}\right| \alpha \phi_{\max }}{1-\left|k^{*}\right| \alpha \phi_{\max }}
$$

Note that this implies that stability can only be expected if $\psi(t)<\left|k^{*-1}\right| \quad \forall t \geq t_{0}$. (b) Let $|v|>u_{0}$. Hence,

$$
u=u_{0} \operatorname{sgn}(v) \text { and }|v|>u_{0}
$$

and the system equations in Theorem 3.2 become

$$
\dot{x}_{p}(t)=A_{m} x_{p}(t)+b_{p} u_{0} \operatorname{sgn}(v(t))-b_{m} \theta^{* T} x_{p}(t) .
$$

Correspondingly,

$$
\begin{aligned}
\dot{W} & =x_{p}^{T}\left[A_{m}^{T} P+P A_{m}^{T}\right] x_{p}-2\left(b_{m}^{T} P x_{p}\right)\left(\theta^{* T} x_{p}\right)+2\left(b_{m}^{T} P x_{p}\right) \frac{u_{0}}{\left|k^{*}\right|} \operatorname{sgn}\left(\frac{v}{k^{*}}\right) \\
& =-x_{p}^{T} Q x_{p}+2\left|b_{m}^{T} P x_{p}\right| u_{0} \operatorname{sgn}\left(\frac{v}{k^{*}}\right) \operatorname{sgn}\left(b_{m}^{T} P x_{p}\right)-2\left(b_{m}^{T} P x_{p}\right)\left(\theta^{* T} x_{p}\right) .
\end{aligned}
$$

The proof is given by considering two separate cases, based on the signs of $v$ and $\left(b_{p}^{T} P x_{p}\right)$. 
Case I $\operatorname{sgn}\left(\frac{v}{k^{*}}\right)=\operatorname{sgn}\left(b_{m}^{T} P x_{p}\right)$. In this case Eq. (A.6) becomes

$$
\dot{W}\left(x_{p}\right)=-x_{p}^{T} Q x_{p}-2\left(b_{m}^{T} P x_{p}\right)\left(\theta^{* T} x_{p}\right)+2\left|b_{m}^{T} P x_{p}\right| \frac{u_{0}}{\left|k^{*}\right|} .
$$

Since $|v|>u_{0}$, we have, after some algebraic manipulations on $\left(b_{m}^{T} P x_{p}\right) \frac{v}{k^{*}}>\left\|b_{m}^{T} P x_{p}\right\| \frac{u_{0}}{\left|k^{*}\right|}$, that

$$
\begin{aligned}
-x_{p}^{T} Q x_{p}-2\left(b_{m}^{T} P x_{p}\right)\left(\theta^{* T} x_{p}\right)+2\left|b_{m}^{T} P x_{p}\right| \frac{u_{0}}{\left|k^{*}\right|} \\
+\left[x_{p}^{T} Q x_{p}-2\left(b_{m}^{T} P x_{p}\right)[\phi+\psi k \theta]^{T} x_{p}-2\left(b_{m} T P x_{p}\right) \frac{k}{k^{*}} r\right]<0 .
\end{aligned}
$$

The definition of $\mathcal{A}$ implies that the term within the bracket is positive so that $\dot{W}_{p}<0 \forall x_{p} \in \mathcal{A}$.

Case II $\operatorname{sgn}\left(\frac{v}{k^{*}}\right)=-\operatorname{sgn}\left(b_{m}^{T} P x_{p}\right)$. Here Eq. (A.6) becomes

$$
\begin{aligned}
\dot{W}\left(x_{p}\right) & =-x_{p}^{T} Q x_{p}-2\left(b_{m}^{T} P x_{p}\right)\left(\theta^{* T} x_{p}\right)-2\left|b_{m}^{T} P x_{p}\right| \frac{u_{0}}{\left|k^{*}\right|} \\
& \leq-q_{0}\left\|x_{p}\right\|^{2}+2\left|b_{m} T P x_{p}\right|\left\|\theta^{*}\right\|\left\|x_{p}\right\|-2\left|b_{m} T P x_{p}\right| \frac{u_{0}}{\left|k^{*}\right|} \\
& \leq 0 \quad \text { if }\left\|x_{p}\right\|<\frac{2 p_{b} u_{0}}{\left|k^{*}\right|\left|2 p_{b}\right|\left|\theta^{*} \|-q_{0}\right|}
\end{aligned}
$$

The upper bound of $\left\|x_{p}\right\|$ in the set $\mathcal{A}$ implies that $\dot{W}<0 \forall x_{p} \in \mathcal{A}$ in this case as well. Finally if $\mathcal{B}$ is defined as

$$
\mathcal{B} \triangleq\left\{x_{p} \mid W\left(x_{p}\right)=\lambda_{\min }(P)\left[\frac{2 p_{b} u_{0}}{\left|k^{*}\right||| 2 p_{b}|| \theta^{*}||-q_{0} \mid}\right]^{2}\right\}
$$

then $\mathcal{B}$ defines a level surface of $W\left(x_{p}\right)$. Condition (ii) in Theorem 3.2 ensures that 
$\mathcal{B} \subset \mathcal{A}$ since

$$
\begin{gathered}
\phi_{\max } \quad<\frac{q_{0}-\rho\left|k^{*}\right| \frac{r_{0}}{u_{0}}\left|2 p_{b}\right|\left|\theta^{*} \|-q_{0}\right|}{2 p_{b}+\alpha\left|k^{*}\right|\left[q_{0}+2 p_{b}\left\|\theta^{*}\right\|\right.} \\
\Rightarrow \frac{2 p_{b} u_{0}}{\left|k^{*}\right|\left|2 p_{b}\right|\left|\theta^{*} \|-q_{0}\right|}>\sqrt{\frac{\lambda_{\max }(P)}{\lambda_{\min }(P)}}\left[\frac{2 p_{b} r_{0}}{q_{0}-\phi_{\max }\left[2 p_{b}+\alpha\left|k^{*}\right|\left(q_{0}+2 p_{b} \theta^{*} \|\right)\right.}\right] .
\end{gathered}
$$

Hence $\dot{W}\left(x_{p}\right)<0$ on all points on the level surface and Theorem 3.2 follows.

\section{Proof of Theorem 3.3}

Define $W(x)=x^{T} P x$ and

$$
\mathcal{A} \triangleq\left\{x \mid \frac{2 p_{b}\left(\left|k^{*}\right|+\phi_{\max }\right) r_{0}}{q_{0}-2 p_{b} \phi_{\max }\|C\|} \leq\|x\| \leq \frac{2 p_{b} u_{0}}{\left|2 p_{b}\left\|\bar{\theta}^{* T} C\right\|-q_{0}\right|}\right\} .
$$

Condition (ii) implies that

$$
\phi_{\max } \leq \frac{q_{0}-\left|k^{*}\right| \rho \frac{r_{0}}{u_{0}}\left|2 p_{b}\left\|\bar{\theta}^{* T} C\right\|-q_{0}\right|}{\rho \frac{r_{0}}{u_{0}}\left|2 p_{b}\left\|\bar{\theta}^{*} C\right\|-q_{0}\right|+2 p_{b}\|C\|} .
$$

As in the proof of Theorem 3.1, two cases are considered.

(a) Let $|v| \leq u_{0}$. Here $\Delta u=0$, and hence

$$
u(t)=v(t)=\theta^{T}(t) \omega(t) .
$$

As a result,

$$
\dot{x}(t)=A_{m n} x(t)+b_{m n}\left[\bar{\phi}^{T}(t) \bar{\omega}(t)+k(t) r(t)\right] .
$$


This leads to a time-derivative

$$
\begin{aligned}
& \dot{W}(x)=x^{T}\left[A_{m n}^{T} P+P A_{m n}^{T}\right] x+2\left(b_{m n}^{T} P x\right)\left[\bar{\phi}^{T}(t) \bar{\omega}(t)+k(t) r(t)\right] \\
& \leq \quad-q_{0}\|x\|^{2}+2 p_{b}\|x\|^{2} \phi_{\max }\|C\|+2 p_{b}\|x\|\left[\left|k^{*}\right|+\phi_{\max }\right] r_{0} \\
& \leq \quad 0 \quad \forall x \in \mathcal{A}
\end{aligned}
$$

which follows from the definition of $\mathcal{A}$ and condition (ii) in Theorem 3.3.

(b) Let $|v|>u_{0}$. Hence,

$$
u=u_{0} \operatorname{sgn}(v) \text { and }|v|>u_{0}
$$

and Eq. (3.21) becomes

$$
\dot{x}(t)=A_{m n} x(t)+b_{m n} u_{0} \operatorname{sgn}(v(t))-b_{m n} \bar{\theta}^{* T} \bar{\omega}(t) .
$$

Correspondingly,

$$
\begin{aligned}
\dot{W} & =x^{T}\left[A_{m n}^{T} P+P A_{m n}^{T}\right] x-2\left(b_{m n}^{T} P x\right)\left(\bar{\theta}^{* T} C x\right)+2\left(b_{m n}^{T} P x\right) u_{0} \operatorname{sgn}(v) \\
& =-x^{T} Q x+2\left|b_{m n}^{T} P x\right| u_{0} \operatorname{sgn}(v) \operatorname{sgn}\left(b_{m n}^{T} P x\right)-2\left(b_{m n}^{T} P x\right)\left(\bar{\theta}^{* T} C x\right) .
\end{aligned}
$$

As in the previous sections, the proof is given by considering two separate cases, based on the signs of $v$ and $\left(b_{m n}^{T} P x\right)$.

Case I $\operatorname{sgn}(v)=\operatorname{sgn}\left(b_{m n}^{T} P x\right)$. In this case Eq. (A.8) becomes

$$
\dot{W}(x)=-x^{T} Q x-2\left(b_{m n}^{T} P x\right)\left(\bar{\theta}^{* T} C x\right)+2\left|b_{m n}^{T} P x\right| u_{0} .
$$

Since $|v|>u_{0}$, it is obtained, after some algebraic manipulations on $\left(b_{m n}^{T} P x\right) v \geq$ $\left|b_{m n}^{T} P x\right| u_{0}$, that

$$
\begin{aligned}
-x^{T} Q x-2 & \left(b_{m n}^{T} P x\right)\left(\bar{\theta}^{* T} \bar{\omega}\right)+2\left|b_{m n}^{T} P x\right| u_{0} \\
+ & {\left[x^{T} Q x-2\left(b_{m n}^{T} P x\right)\left(\bar{\phi}^{T} \bar{\omega}\right)-2\left(b_{m n}^{T} P x\right) k r\right] \leq 0 }
\end{aligned}
$$


The definition of $\mathcal{A}$ implies that the term within the bracket is non-negative so that $\dot{W}_{p} \leq 0 \forall x \in \mathcal{A}$.

Case II $\operatorname{sgn}(v)=-\operatorname{sgn}\left(b_{m n}^{T} P x\right)$. Here Eq. (A.8) becomes

$$
\begin{aligned}
\dot{W}(x) & =-x^{T} Q x-2\left(b_{m n}^{T} P x\right)\left(\bar{\theta}^{* T} C x\right)-2\left|b_{m n}^{T} P x\right| u_{0} \\
& \leq-q_{0}\|x\|^{2}+2\left|b_{m n}^{T} P x\right|\left\|\bar{\theta}^{* T} C\right\|\|x\|-2\left|b_{m n}^{T} P x\right| u_{0} \\
& \leq 0 \quad \text { if }\|x\|<\frac{2 p_{b} u_{0}}{\left|2 p_{b}\left\|\bar{\theta}^{* T} C\right\|-q_{0}\right|} .
\end{aligned}
$$

The upper bound of $\|x\|$ in the set $\mathcal{A}$ implies that $\dot{W} \leq 0 \forall x \in \mathcal{A}$ in this case as well. Finally if a level surface of $W(x)$ is defined as

$$
\mathcal{B} \triangleq\left\{x \mid W(x)=\lambda_{\min }(P)\left[\frac{2 p_{b} u_{0}}{\left|2 p_{b}\left\|\bar{\theta}^{* T} C\right\|-q_{0}\right|}\right]^{2}\right\}
$$

condition (ii) in Theorem 3.3 ensures that $\mathcal{B} \subset \mathcal{A}$ since

$$
\begin{aligned}
\phi_{\max } & \leq \frac{q_{0}-\left|k^{*}\right| \rho \frac{r_{0}}{u_{0}}\left|q_{0}-2 p_{b}\left\|\bar{\theta}^{* T} C\right\|\right|}{\rho \frac{r_{0}}{u_{0}}\left|q_{0}-2 p_{b}\right|\left|\bar{\theta}^{* T} C\left\|\mid+2 p_{b}\right\| C \|\right.} \\
\Rightarrow \frac{2 p_{b} u_{0}}{\left|2 p_{b}\left\|\bar{\theta}^{* T} C\right\|-q_{0}\right|} & \geq \sqrt{\frac{\lambda_{\max }(P)}{\lambda_{\min }(P)}}\left[\frac{2 p_{b}\left(\left|k^{*}\right|+\phi_{\max }\right) r_{0}}{q_{0}-2 p_{b} \phi_{\max }\|C\|}\right] .
\end{aligned}
$$

Hence $\dot{W}(x) \leq 0$ on all points on the level surface and Theorem 3.3 follows.

\section{Proof of Theorem 3.4}

Define $W(x)=x^{T} P x$ and

$$
\mathcal{A} \triangleq\left\{x \mid \frac{2 p_{b}\left(\left|k^{*}\right| r_{0}+\nu_{0}+\phi_{\max } r_{0}\right)}{q_{0}-2 p_{b} \phi_{\max }\|C\|} \leq\|x\| \leq \frac{2 p_{b}\left(u_{0}-\nu_{0}\right)}{\left|2 p_{b}\left\|\bar{\theta}^{* T} C\right\|-q_{0}\right|}\right\}
$$


Condition (ii) implies that

$$
\phi_{\max } \leq \frac{q_{0}-\rho \frac{\left|k^{*}\right| r_{0}+\nu_{0}}{u_{0}-\nu_{0}}\left|2 p_{b}\left\|\bar{\theta}^{* T} C\right\|-q_{0}\right|}{\rho \frac{r_{0}}{u_{0}-\nu_{0}}\left|2 p_{b}\left\|\bar{\theta}^{* T} C\right\|-q_{0}\right|+2 p_{b}\|C\|} .
$$

As in the proof of Theorem 3.3, two cases are considered.

(a) Let $|v| \leq u_{0}$. Here $\Delta u=0$, and hence

$$
u_{c}(t)=v(t)=\theta^{T}(t) \omega(t) .
$$

As a result,

$$
\dot{x}(t)=A_{m n} x(t)+b_{m n}\left[\bar{\phi}^{T}(t) \bar{\omega}(t)+k(t) r(t)+\nu(t)\right] .
$$

This leads to a time-derivative

$$
\begin{aligned}
& \dot{W}(x)=x^{T}\left[A_{m n}^{T} P+P A_{m n}^{T}\right] x+2\left(b_{m n}^{T} P x\right)\left[\bar{\phi}^{T}(t) \bar{\omega}(t)+k(t) r(t)+\nu(t)\right] \\
& \leq \quad-q_{0}\|x\|^{2}+2 p_{b}\|x\|^{2} \phi_{\max }\|C\|+2 p_{b}\|x\|\left[\left|k^{*}\right| r_{0}+\nu_{0}+\phi_{\max } r_{0}\right] \\
& \leq \quad 0 \quad \forall x \in \mathcal{A}
\end{aligned}
$$

which follows from the definition of $\mathcal{A}$ and condition (ii) in Theorem 3.4.

(b) Let $|v|>u_{0}$. Hence,

$$
u_{c}=u_{0} \operatorname{sgn}(v) \text { and }|v|>u_{0}
$$

and Eq. (3.21) becomes

$$
\dot{x}(t)=A_{m n} x(t)+b_{m n}\left(u_{0} \operatorname{sgn}(v(t))+\nu(t)\right)-b_{m n} \bar{\theta}^{* T} \bar{\omega}(t) .
$$


Correspondingly,

$$
\begin{aligned}
\dot{W} & =x^{T}\left[A_{m n}^{T} P+P A_{m n}^{T}\right] x-2\left(b_{m n}^{T} P x\right)\left(\bar{\theta}^{* T} C x\right)+2\left(b_{m n}^{T} P x\right) \operatorname{sgn}(v)\left(u_{0}+\nu \operatorname{sgn}(v)\right) \\
& =-x^{T} Q x+2\left|b_{m n}^{T} P x\right| \operatorname{sgn}(v)\left(u_{0}+\nu \operatorname{sgn}(v)\right) \operatorname{sgn}\left(b_{m n}^{T} P x\right)-2\left(b_{m n}^{T} P x\right)\left(\bar{\theta}^{* T} C x\right) .
\end{aligned}
$$

As in the previous sections, the proof is given by considering two separate cases, based on the signs of $v$ and $\left(b_{m n}^{T} P x\right)$. It is noted that as $\nu_{0}<u_{0}$ then $\operatorname{sgn}(v)=$ $\operatorname{sgn}\left(u_{0} \operatorname{sgn}(v)+\nu\right)$ when $|v|>u_{0}$.

Case I $\operatorname{sgn}(v)=\operatorname{sgn}\left(b_{m n}^{T} P x\right)$. In this case Eq. (A.10) becomes

$$
\dot{W}(x)=-x^{T} Q x-2\left(b_{m n}^{T} P x\right)\left(\bar{\theta}^{* T} C x\right)+2\left|b_{m n}^{T} P x\right|\left[u_{0}+\nu \operatorname{sgn}(v)\right] .
$$

Since $|v|>u_{0}$, it is obtained, after some algebraic manipulations on $\left(b_{m n}^{T} P x\right)(v+\nu) \geq$ $\left|b_{m n}^{T} P x\right|\left(u_{0}+\nu \operatorname{sgn}(v)\right)$, that

$$
\begin{aligned}
-x^{T} Q x- & 2\left(b_{m n}^{T} P x\right)\left(\bar{\theta}^{* T} \bar{\omega}\right)+2\left|b_{m n}^{T} P x\right|\left(u_{0}+\nu \operatorname{sgn}(v)\right) \\
& +\left[x^{T} Q x-2\left(b_{m n}^{T} P x\right)\left(\bar{\phi}^{T} \bar{\omega}\right)-2\left(b_{m n}^{T} P x\right)(k r+\nu)\right] \leq 0
\end{aligned}
$$

The definition of $\mathcal{A}$ implies that the term within the bracket is non-negative so that $\dot{W}_{p} \leq 0 \forall x \in \mathcal{A}$.

Case II $\operatorname{sgn}(v)=-\operatorname{sgn}\left(b_{m n}^{T} P x\right)$. Here Eq. (A.10) becomes

$$
\begin{aligned}
\dot{W}(x) & =-x^{T} Q x-2\left(b_{m n}^{T} P x\right)\left(\bar{\theta}^{* T} C x\right)-2\left|b_{m n}^{T} P x\right|\left(u_{0}+\nu \operatorname{sgn}(v)\right) \\
& \leq-q_{0}\|x\|^{2}+2\left|b_{m n}^{T} P x\right|\left\|\bar{\theta}^{* T} C\right\|\|x\|-2\left|b_{m n}^{T} P x\right|\left(u_{0}-\nu_{0}\right) \\
& \leq 0 \quad \text { if }\|x\|<\frac{2 p_{b}\left(u_{0}-\nu_{0}\right)}{\left|2 p_{b}\left\|\bar{\theta}^{* T} C\right\|-q_{0}\right|} .
\end{aligned}
$$

The upper bound of $\|x\|$ in the set $\mathcal{A}$ implies that $\dot{W} \leq 0 \forall x \in \mathcal{A}$ in this case as 
well. Finally if a level surface of $W(x)$ is defined as

$$
\mathcal{B} \triangleq\left\{x \mid W(x)=\lambda_{\min }(P)\left[\frac{2 p_{b}\left(u_{0}-\nu_{0}\right)}{\left|2 p_{b}\left\|\bar{\theta}^{* T} C\right\|-q_{0}\right|}\right]^{2}\right\}
$$

condition (ii) in Theorem 3.3 ensures that $\mathcal{B} \subset \mathcal{A}$ since

$$
\begin{aligned}
\phi_{\max } & \leq \frac{q_{0}-\rho \frac{\left|k^{*}\right| r_{0}+\nu_{0}}{u_{0}-\nu_{0}}\left|q_{0}-2 p_{b}\left\|\bar{\theta}^{* T} C\right\|\right|}{\rho \frac{r_{0}}{u_{0}-\nu_{0}}\left|q_{0}-2 p_{b}\left\|\bar{\theta}^{* T} C\right\|\right|+2 p_{b}\|C\|} \\
\Rightarrow \frac{2 p_{b}\left(u_{0}-\nu_{0}\right)}{\left|2 p_{b}\right|\left|\bar{\theta}^{* T} C \|-q_{0}\right|} & \geq \sqrt{\frac{\lambda_{\max }(P)}{\lambda_{\min }(P)}}\left[\frac{2 p_{b}\left(\left|k^{*}\right| r_{0}+\nu_{0}+\phi_{\max } r_{0}\right)}{q_{0}-2 p_{b} \phi_{\max }\|C\|}\right] .
\end{aligned}
$$

Hence $\dot{W}(x) \leq 0$ on all points on the level surface and Theorem 3.4 follows. 


\section{Appendix B}

\section{Proofs for Theorems in Chapter 4}

\section{Lemma B.1}

Lemma B.1 If an input-output pair $u$ and $\phi$ are related by the regression equation

$$
u(t)=\bar{\phi}^{T}(t) \theta_{0}
$$

and the unknown parameter $\theta_{0}$ is estimated using the update law

$$
\theta(t)=\theta(t-1)+\frac{a \bar{\phi}(t-d)}{c+\bar{\phi}^{T}(t-d) \bar{\phi}(t-d)}\left[u(t-d)-\bar{\phi}^{T}(t-d) \theta(t-1)\right]
$$

where $d \geq 1$ is the pure time delay. Then (i) $\|\tilde{\theta}(t)\|$ is nonincreasing, and (ii) $\|\tilde{\theta}(t)\| \leq$ $\left\|\tilde{\theta}\left(t_{0}\right)\right\|$.

Proof: Defining the parameter error $\tilde{\theta}(t)=\theta(t)-\theta_{0}$, and a Lyapunov function candidate $V(t)=\|\tilde{\theta}(t)\|^{2}$, it is obtained that

$$
\tilde{\theta}(t)=\tilde{\theta}(t-1)-a \frac{\bar{\phi}(t-1)}{c+\bar{\phi}^{T}(t-1) \bar{\phi}(t-1)}\left[\bar{\phi}^{T}(t-1) \tilde{\theta}(t-1)\right]
$$

and hence,

$$
V(t)-V(t-1)=a\left[-2+\frac{a \bar{\phi}^{T}(t-1) \bar{\phi}(t-1)}{c+\bar{\phi}^{T}(t-1) \bar{\phi}(t-1)}\right] \frac{\left[\bar{\phi}^{T}(t-1) \tilde{\theta}(t-1)\right]^{2}}{c+\bar{\phi}^{T}(t-1) \bar{\phi}(t-1)}
$$




$$
\leq 0 \quad \text { if } 0<a<2 \text { and } c>0
$$

Hence, (i) and (ii) in Lemma B.1 follows.

\section{Proof of Theorem 4.1}

Define $W(t)=y^{2}(t), \Delta W(t)=W(t)-W(t-1)$, and $\mathcal{A} \triangleq\left\{y \mid y_{\min } \leq y \leq y_{\max }\right\}$ where

$$
\begin{aligned}
& y_{\text {min }}=\frac{1+\mid b_{p}\left\|\tilde{\theta}\left(t_{0}\right)\right\|}{1-\left|a_{m}\right|-\left|b_{p}\right|\left|\tilde{\theta}\left(t_{0}\right)\right| \mid}\left|b_{m}\right| r_{0} \\
& y_{\max }=\frac{\left|b_{p} u_{0}\right|}{\left|a_{p}\right|-1} .
\end{aligned}
$$

It is noted that condition (i) in Theorem 4.1 implies that $\mathcal{A}$ is non-empty. The proof is given by showing that

$$
|y(t)| \leq y_{\max } \quad \text { if }\left|y\left(t_{0}\right)\right| \leq y_{\max }
$$

This is established by showing that

$$
\Delta W(t) \leq 0 \quad \text { if } y(t) \in \mathcal{A}
$$

and

$$
W(t+1) \leq y_{\max }^{2} \quad \text { if }|y(t)| \leq y_{\min }
$$

It is easy to see that (I) and (II) imply Eq. (B.3). Since

$$
\Delta W(t+1)=b_{p}^{2}\left[u(t)+\frac{a_{p}}{b_{p}} y(t)\right]^{2}-y^{2}(t),
$$


defining $z(t)$ as

$$
z(t)=\left|b_{p}\right|\left|u(t)+\frac{a_{p}}{b_{p}} y(t)\right|
$$

(I) follows if it can be shown that

$$
|y(t)| \geq z(t) \quad \forall y(t) \in \mathcal{A} .
$$

Also, Eq. (II) follows if the following inequality can be established:

$$
z(t) \leq y_{\min } \quad \text { if }|y(t)| \leq y_{\min } .
$$

The proof is completed by considering three separate cases and showing in each case that $z(t)$ satisfies the inequalities (B.4) and (B.5).

Case (a) $|v| \leq u_{0}$.

Since $u(t)=v(t)$ and the latter can be expressed as

$$
v(t)=-\theta_{0 y} y(t)-\tilde{\theta}_{y}(t) y(t)+\theta_{0 u} u(t)+\tilde{\theta}_{u}(t) u(t)
$$

it is obtained that

$$
\begin{aligned}
z(t) & =\left|b_{p}\right|\left|\frac{a_{m}}{b_{p}} y(t)+\frac{b_{m}}{b_{p}} r(t)-\tilde{\theta}_{y}(t) y(t)+\tilde{\theta}_{u}(t) b_{m} r(t)\right| \\
& \leq\left(\left|a_{m}\right|+\left|b_{p}\right|\left\|\tilde{\theta}\left(t_{0}\right)\right\|\right)|y|+\left(1+\left|b_{p}\right|\left\|\tilde{\theta}\left(t_{0}\right)\right\|\right)\left|b_{m}\right| r_{0} .
\end{aligned}
$$

If $|y(t)| \geq y_{\min }$, it follows that (B.6) can be simplified as $z(t) \leq|y(t)|$ and hence, (B.4) holds. If $|y(t)| \leq y_{\min }$, by direct substitution in Eq. (B.6), it follows that

$$
z(t) \leq y_{\min } \text { if }|y(t)| \leq y_{\min }
$$

and hence, (B.5) holds.

Case (b) $|v|>u_{0}$ and $\operatorname{sgn}(v)=\operatorname{sgn}\left(\frac{a_{p}}{b_{p}} y\right)$. 
In this case,

$$
z(t)=\left|b_{p}\right|\left|u(t)+\frac{a_{p}}{b_{p}} y(t)\right| \leq\left|b_{p}\right|\left|v(t)+\frac{a_{p}}{b_{p}} y(t)\right|
$$

which reduces to the inequality (B.6), and hence, as in case (a), (B.4) and (B.5) hold. Case (c) $|v|>u_{0}$ and $\operatorname{sgn}(v)=-\operatorname{sgn}\left(\frac{a_{p}}{b_{p}} y\right)$.

This results in

$$
z(t)=\left|b_{p}\right|\left|u_{0}-\right| \frac{a_{p}}{b_{p}} y(t)||
$$

and two subcases evolve.

$\underline{\text { Subcase (i) }} u_{0} \geq\left|\frac{a_{p}}{b_{p}} y\right|$. Here,

$$
\begin{aligned}
z(t)=\left|b_{p}\right|\left|u_{0}-\right| \frac{a_{p}}{b_{p}} y(t)|| & =\left|b_{p}\right|\left[u_{0}-\left|\frac{a_{p}}{b_{p}} y(t)\right|\right] \\
& \leq\left|b_{p}\right|\left[|v(t)|-\left|\frac{a_{p}}{b_{p}} y(t)\right|\right] .
\end{aligned}
$$

Since $\operatorname{sgn}(v)=-\operatorname{sgn}\left(\frac{a_{p}}{b_{p}} y\right)$, in this subcase then

$$
z(t) \leq\left|b_{p}\right|\left|v(t)+\frac{a_{p}}{b_{p}} y(t)\right|
$$

As in cases (a) and (b), this implies that (B.4) and (B.5) hold.

Subcase (ii) $u_{0}<\left|\frac{a_{p}}{b_{p}} y\right|$. Unlike all the cases above, it is required that $y(t)$ be bounded above by $y_{\max }$ to establish the necessary inequalities. It is noted that

$$
\begin{aligned}
z(t)=\left|b_{p}\right|\left|u_{0}-\right| \frac{a_{p}}{b_{p}} y(t)|| & =\left|b_{p}\right|\left[\left|\frac{a_{p}}{b_{p}} y(t)\right|-u_{0}\right] \\
& \leq|y(t)| \quad \text { if } y(t) \leq y_{\max }
\end{aligned}
$$

and hence (B.4) holds. Since the inequality in (B.7) holds for any $y(t)$ such that $|y(t)| \leq y_{\max }$, it follows that (B.5) holds as well.

Since the cases considered above are mutually exclusive and collectively exhaustive, it has been established that (I) and (II) follow in all cases. Hence the proof. 


\section{Proof of Theorem 4.2}

The vector $\psi_{y} \in \mathbb{R}^{n_{d}}$ can be viewed as a state vector for the plant with the description

$$
\psi_{y}(t+1)=A_{m} \psi_{y}(t)-\beta_{0} b_{m}\left[u(t)-\psi_{y}^{T}(t) C \theta_{0 y}-\psi_{u}^{T}(t) \theta_{0 u}\right]
$$

where

$$
C=\left[\begin{array}{c}
0_{(d-1) \times n} \\
I_{n \times n}
\end{array}\right], \quad A_{m}=\left[\begin{array}{ccc}
0 & \cdots & 0 \\
& I_{\left(n_{d}-1\right) \times\left(n_{d}-1\right)} & \vdots \\
& & 0
\end{array}\right], \quad b_{m}=\left[\begin{array}{c}
1 \\
0 \\
\vdots \\
0
\end{array}\right] .
$$

It can easily be shown that

$$
A_{m}^{T} P A_{m}-P=-I, \quad b_{m}^{T} P A_{m}=0, \quad b_{m}^{T} P b_{m}=n_{d} .
$$

Now is 'hyper-shell' $\mathcal{A}$, in $\mathbb{R}^{n_{d}}$ defined as

$$
\mathcal{A} \triangleq\left\{\psi \mid \psi_{\min } \leq\|\psi\| \leq \psi_{\max }\right\}
$$

where

$$
\psi_{\min }=\frac{\sqrt{m_{d}} u_{0} \tilde{\theta}_{\max }+\left(\frac{1}{\left|\beta_{0}\right|}+\tilde{\theta}_{\max }\right) y_{\max }^{*}}{\frac{1}{\sqrt{n_{d}}\left|\beta_{0}\right|}-\tilde{\theta}_{\max }}, \quad \psi_{\max }=\frac{u_{0}\left(1-\left\|\theta_{0 u}\right\|_{1}\right)}{\left\|\theta_{0 y}\right\|-\frac{1}{\sqrt{n_{d}}\left|\beta_{0}\right|}} .
$$

Let a scalar quantity $W(t) \triangleq \psi_{y}^{T}(t) P \psi_{y}(t)$, and $\Delta W(t)=W(t)-W(t-1)$. It 
will be shown below that equations (I) and (II) given by

$$
\begin{gathered}
\Delta W \leq 0 \quad \text { if } \psi_{y}(t) \in \mathcal{A} \\
W(t+1) \leq n_{d} \psi_{\min }^{2} \leq \psi_{\max }^{2} \quad \text { if }\left\|\psi_{y}(t)\right\| \leq \psi_{\min }
\end{gathered}
$$

hold. It is noted that (I) and (II) imply that $W(t) \leq \psi_{\max }^{2}$ for all $W\left(t_{0}\right) \leq \psi_{\max }^{2}$ which proves the theorem.

From Eqs. (B.8) and (B.9), $\Delta W(t)$ is given by

$$
\Delta W(t)=-\left\|\psi_{y}(t)\right\|^{2}+n_{d} \beta_{0}^{2}\left[u(t)-\psi_{y}^{T}(t) C \theta_{0 y}-\psi_{u}^{T}(t) \theta_{0 u}\right]^{2}
$$

If a nonnegative quantity $z(t)$ is defined as

$$
z(t)=\sqrt{n_{d}}\left|\beta_{0}\right|\left|u(t)-\psi_{y}^{T}(t) C \theta_{0 y}-\psi_{u}^{T}(t) \theta_{0 u}\right|
$$

then (I) follows if

$$
z(t) \leq\left\|\psi_{y}(t)\right\| \quad \text { if } \psi_{y}(t) \in \mathcal{A} \text {. }
$$

Also,

$$
\begin{aligned}
& W(t)=\psi_{y}^{T}(t)[P-I] \psi_{y}^{T}(t)+n_{d} \beta_{0}^{2}\left[u(t)-\psi_{y}^{T}(t) C \theta_{0 y}-\psi_{u}^{T}(t) \theta_{0 u}\right]^{2} \\
& \leq\left(n_{d}-1\right) \psi_{\min }^{2}+n_{d} \beta_{0}^{2}\left[u(t)-\psi_{y}^{T}(t) C \theta_{0 y}-\psi_{u}^{T}(t) \theta_{0 u}\right]^{2} \\
& \text { if }\left\|\psi_{y}(t)\right\| \leq \psi_{\min } .
\end{aligned}
$$

Hence, Eq. (II) is satisfied if

$$
z(t) \leq \psi_{\min } \quad \text { if }\left\|\psi_{y}(t)\right\| \leq \psi_{\min }
$$

As in the proof of Theorem 4.1, three cases are considered below, in each of which, it is shown that Eq. (B.11) and (B.12) hold, which suffices to establish the proof. Case (a) $|v| \leq u_{0}$. 
Since $u(t)=v(t)$, it is obtained that

$$
u(t)=\psi_{y}^{T}(t) C \theta_{0 y}+\psi_{u}^{T}(t) \theta_{0 u}+\psi_{y}^{T}(t) C \tilde{\theta}_{y}(t)+\psi_{u}^{T}(t) \tilde{\theta}_{u}(t)+y^{*}(t+d)\left(\theta_{0 r}+\tilde{\theta}_{r}(t)\right) .
$$

This simplifies $z(t)$ which becomes

$$
\begin{aligned}
z(t) & =\sqrt{n_{d}}\left|\beta_{0}\right|\left|\psi_{y}^{T}(t) C \tilde{\theta}_{y}(t)+\psi_{u}^{T}(t) \tilde{\theta}_{u}(t)+y^{*}(t+d)\left(\theta_{0 r}+\tilde{\theta}_{r}(t)\right)\right| \\
& \leq \sqrt{n_{d}}\left|\beta_{0}\right|||\left|\psi_{y}(t)\right|\left|\tilde{\theta}_{\max }+\sqrt{m_{d}} u_{0} \tilde{\theta}_{\max }+\left(\frac{1}{\left|\beta_{0}\right|}+\tilde{\theta}_{\max }\right) y_{\max }^{*}\right|
\end{aligned}
$$

If $\left\|\psi_{y}(t)\right\| \geq \psi_{\min }$, then Eq. (B.13) can be simplified further as

$$
z(t) \leq\left\|\psi_{y}(t)\right\|
$$

and hence Eq. (B.11) holds. If $\left\|\psi_{y}(t)\right\| \leq \psi_{\min }$, by direct substitution in Eq. (B.13), it follows that $z(t) \leq \psi_{\min }$ and hence Eq. (B.12) holds.

Case (b) $|v| \leq u_{0}$ and $\operatorname{sgn}(v)=\operatorname{sgn}\left(-\psi_{y}^{T}(t) C \theta_{0 y}-\psi_{u}^{T}(t) \theta_{0 u}\right)$.

Then

$$
z(t) \leq \sqrt{n_{d}}\left|\beta_{0}\right|\left|v(t)-\psi_{y}^{T}(t) C \theta_{0 y}-\psi_{u}^{T}(t) \theta_{0 u}\right|
$$

Since Eq. (B.14) is similar to Eq. (B.13) in case (a), same arguments as above can be used to conclude (B.11) and (B.12).

Case (c) $|v| \leq u_{0}$ and $\operatorname{sgn}(v)=\operatorname{sgn}\left(\psi_{y}^{T} C \theta_{0 y}+\psi_{u}^{T} \theta_{0 u}\right)$.

Here,

$$
\left|u(t)-\psi_{y}^{T}(t) C \theta_{0 y}-\psi_{u}^{T}(t) \theta_{0 u}\right|=\left|u_{0}-\right| \psi_{y}^{T}(t) C \theta_{0 y}+\psi_{u}^{T}(t) \theta_{0 u}||
$$

and two subcases evolve. 
$\underline{\text { Subcase (i) }} u_{0} \geq\left|\psi_{y}^{T} C \theta_{0 y}+\psi_{u}^{T} \theta_{0 u}\right|$. Here,

$$
\begin{aligned}
z(t) & =\sqrt{n_{d}}\left|\beta_{0}\right|\left[u_{0}-\left|\psi_{y}^{T}(t) C \theta_{0 y}+\psi_{u}^{T}(t) \theta_{0 u}\right|\right] \\
& \leq \sqrt{n_{d}}\left|\beta_{0}\right|\left[|v|-\left|\psi_{y}^{T}(t) C \theta_{0 y}+\psi_{u}^{T}(t) \theta_{0 u}\right|\right] .
\end{aligned}
$$

Since $\operatorname{sgn}(v)=\operatorname{sgn}\left(-\psi_{y}^{T} C \theta_{0 y}-\psi_{u}^{T} \theta_{0 u}\right)$, we have that

$$
z(t) \leq \sqrt{n_{d}}\left|\beta_{0}\right|\left|v-\psi_{y}^{T}(t) C \theta_{0 y}-\psi_{u}^{T}(t) \theta_{0 u}\right|
$$

once again, this reduces to a form similar to Eq. (B.13) and hence (B.11) and (B.12) hold.

Subcase (ii) $u_{0} \leq\left|\psi_{y}^{T} C \theta_{0 y}+\psi_{u}^{T} \theta_{0 u}\right|$. As in the proof of Theorem 4.1, this is the occasion to use the upper bound on $\left\|\psi_{y}\right\|$ in this subcase. Here, $z(t)$ can be expressed as

$$
\begin{aligned}
z(t) & =\sqrt{n_{d}}\left|\beta_{0}\right|\left[\left|\psi_{y}^{T} C \theta_{0 y}+\psi_{u}^{T} \theta_{0 u}\right|-u_{0}\right] \\
& \leq\left\|\psi_{y}(t)\right\| \text { if } \psi_{y}(t) \leq \psi_{\max }
\end{aligned}
$$

and hence (B.11) holds. Since the inequality in Eq. (B.15) holds for any $\psi_{y}(t)$ such that $\left\|\psi_{y}(t)\right\| \leq \psi_{\max }$, Eq. (B.12) is valid too.

Cases (a), (b), and (c) cover all possible situations and hence the theorem is proved.

\section{Proof of Theorem 4.3}

The vector $\psi_{y} \in \mathbb{R}^{n_{d}}$ defined as in Eq. (4.14) satisfies the description

$$
\psi_{y}(t+1)=A_{m} \psi_{y}(t)-\beta_{0} b_{m}\left[u_{c}(t)+\nu(t)-\psi_{y}^{T}(t) C \theta_{0 y}-\psi_{u_{c}}^{T}(t) \theta_{0 u}-\psi_{\nu}^{T}(t)\left(B_{\beta_{c}}\right] 6\right)
$$


where

$$
\begin{aligned}
\psi_{u_{c}}^{T}(t) & =\left[-u_{c}(t-1),-u_{c}(t-2), \ldots,-u_{c}(t-m-d+1)\right] \\
\psi_{\nu}^{T}(t) & =[-\nu(t-1),-\nu(t-2), \ldots,-\nu(t-m-d+1)],
\end{aligned}
$$

and

$$
C=\left[\begin{array}{c}
0_{(d-1) \times n} \\
I_{n \times n}
\end{array}\right], \quad A_{m}=\left[\begin{array}{ccc}
0 & \cdots & 0 \\
& I_{\left(n_{d}-1\right) \times\left(n_{d}-1\right)} & \vdots \\
& & 0
\end{array}\right], \quad b_{m}=\left[\begin{array}{c}
1 \\
0 \\
\vdots \\
0
\end{array}\right]
$$

As in Theorem 4.2, it can be shown that

$$
A_{m}^{T} P A_{m}-P=-I \quad b_{m}^{T} P A_{m}=0 \quad b_{m}^{T} P b_{m}=n_{d} .
$$

A hypershell $\mathcal{A}$ is once again defined as

$$
\mathcal{A} \triangleq\left\{\psi_{y} \mid \psi_{\min } \leq\left\|\psi_{y}\right\| \leq \psi_{\max }\right\}
$$

with

$$
\psi_{\min }=\frac{\sqrt{m_{d}} u_{0} \tilde{\theta}_{\max }+\bar{\nu}_{0}+\left(\left|\theta_{0 r}\right|+\tilde{\theta}_{\max }\right) y_{\max }^{*}}{\frac{1}{\sqrt{n_{d}}\left|\beta_{0}\right|}-\tilde{\theta}_{\max }} \quad \psi_{\max }=\frac{u_{0}\left(1-\left\|\theta_{0 u}\right\|_{1}\right)-\bar{\nu}_{0}}{\left\|\theta_{0 y}\right\|-\frac{1}{\sqrt{n_{d}}\left|\beta_{0}\right|}}
$$

and $W(t)=\psi_{y}^{t}(t) P \psi_{y}(t)$.

The boundedness of all the signals will be shown by demonstrating that

$$
W(t) \leq \psi_{\max }^{2} \quad \forall W\left(t_{0}\right) \leq \psi_{\max }^{2} .
$$

This in turn is proved by showing that the equations

$$
\Delta W(t) \leq 0 \text { if } \psi_{y}(t) \in \mathcal{A}
$$


and

$$
W(t+1) \leq n_{d} \psi_{\min }^{2} \leq \psi_{\max }^{2} \text { if }\left\|\psi_{y}(t)\right\| \leq \psi_{\min }
$$

hold. If $z(t)$ is defined as

$$
z(t) \triangleq \sqrt{n_{d}}\left|\beta_{0}\right|\left|u_{c}+\nu-\psi_{y}^{T} C \theta_{0 y}-\psi_{u_{c}}^{T} \theta_{0 u}-\psi_{\nu}^{T} \theta_{0 u}\right|
$$

we can show that $\Delta W(t)$ is given by

$$
\Delta W(t)=-\left\|\psi_{y}(t)\right\|^{2}+z^{2}(t)
$$

Hence, Eq. (I) holds if

$$
\left\|\psi_{y}\right\| \geq z(t) \text { if } \psi_{y}(t) \in \mathcal{A} \text {. }
$$

Also, $W(t)$ can be expressed as

$$
W(t)=\psi_{y}^{T}(t)[P-I] \psi_{y}^{T}(t)+z^{2}(t)
$$

Therefore, Eq. (II) is valid if

$$
z(t) \leq \psi_{\min } \quad \text { if }\left\|\psi_{y}(t)\right\| \leq \psi_{\min } .
$$

As in Theorems 4.1 and 4.2, we proceed to establish Theorem 4.3 by showing in three distinct cases that Eqs. (B.17) and (B.18) hold.

Case (a) $|v| \leq u_{0}$.

Since $u_{c}(t)=v(t)$, then

$$
u_{c}(t)=\psi_{y}^{T}(t) C \theta_{0 y}+\psi_{u_{c}}^{T}(t) \theta_{0 u}+\psi_{y}^{T}(t) C \tilde{\theta}_{y}(t)+\psi_{u_{c}}^{T}(t) \tilde{\theta}_{u}(t)+y^{*}(t+d)\left(\theta_{0 r}+\tilde{\theta}_{r}(t)\right) .
$$


This makes it possible to simplify $z(t)$ as

$$
\begin{aligned}
& z(t) \\
& =\sqrt{n_{d}}\left|\beta_{0}\right|\left|\psi_{y}^{T}(t) C \tilde{\theta}_{y}(t)+\psi_{u}^{T}(t) \tilde{\theta}_{u}(t)+y^{*}(t+d)\left(\theta_{0 r}+\tilde{\theta}_{r}(t)\right)+\nu(t)-\psi_{\nu}^{T}(t) \theta_{0 u}\right| \\
& \leq \sqrt{n_{d}}\left|\beta_{0}\right|\left|\left\|\psi_{y}(t)\right\| \tilde{\theta}_{\max }+\sqrt{m_{d}} u_{0} \tilde{\theta}_{\max }+\left(\frac{1}{\left|\beta_{0}\right|}+\tilde{\theta}_{\max }\right) y_{\max }^{*}+\bar{\nu}_{0}\right| .
\end{aligned}
$$

If $\left\|\psi_{y}(t)\right\| \geq \psi_{\min }$, Eq. (B.19) implies that

$$
z(t) \leq\left\|\psi_{y}(t)\right\| \quad \text { if }\left\|\psi_{y}(t)\right\| \in \mathcal{A}
$$

i.e., Eq. (B.17) holds. If $\left\|\psi_{y}(t)\right\| \leq \psi_{\min }$, it is easy to that Eq. (B.18) holds, which completes the proof in this case.

Case (b) $|v| \leq u_{0}$ and $\operatorname{sgn}(v)=\operatorname{sgn}\left(\nu-\psi_{y}^{T} C \theta_{0 y}-\psi_{u_{c}}^{T} \theta_{0 u}-\psi_{\nu}^{T} \theta_{0 u}\right)$.

In this case, it is obtained that

$$
z(t)=\sqrt{n_{d}}\left|\beta_{0}\right|\left|v(t)+\nu(t)-\psi_{y}(t)^{T} C \theta_{0 y}-\psi_{u_{c}}^{T}(t) \theta_{0 u}-\psi_{\nu}^{T}(t) \theta_{0 u}\right| .
$$

Since the right hand sides of Eqs. (B.20) and (B.19) are identical, the same arguments as in case (a) can be used here as well to complete the proof.

Case (c) $|v| \leq u_{0}$ and $\operatorname{sgn}(v)=\operatorname{sgn}\left(-\nu+\psi_{y}^{T} C \theta_{0 y}+\psi_{u_{c}}^{T} \theta_{0 u}+\psi_{\nu}^{T} \theta_{0 u}\right)$.

Here, $z(t)$ can be expressed as

$$
z(t)=\left|u_{0}-\right|-\nu(t)+\psi_{y}^{T}(t) C \theta_{0 y}+\psi_{u_{c}}^{T}(t) \theta_{0 u}+\psi_{\nu}^{T}(t) \theta_{0 u}||
$$

and two subcases evolve.

Subcase (i) $u_{0} \geq\left|-\nu+\psi_{y}^{T} C \theta_{0 y}+\psi_{u_{c}}^{T} \theta_{0 u}+\psi_{\nu}^{T} \theta_{0 u}\right|$.

Then

$$
\begin{aligned}
z(t) & =\sqrt{n_{d}}\left|\beta_{0}\right|\left[u_{0}-\left|-\nu+\psi_{y}^{T} C \theta_{0 y}+\psi_{u_{c}}^{T} \theta_{0 u}+\psi_{\nu}^{T} \theta_{0 u}\right|\right] \\
& \leq \sqrt{n_{d}}\left|\beta_{0}\right|\left[|v|-\left|-\nu+\psi_{y}^{T} C \theta_{0 y}+\psi_{u_{c}}^{T} \theta_{0 u}+\psi_{\nu}^{T} \theta_{0 u}\right|\right] \\
& \leq \sqrt{n_{d}}\left|\beta_{0}\right|\left|v+\nu-\psi_{y}^{T} C \theta_{0 y}-\psi_{u_{c}}^{T} \theta_{0 u}-\psi_{\nu}^{T} \theta_{0 u}\right|
\end{aligned}
$$


Eq. (B.21) is similar to Eq. (B.19) and hence, (I) and (II) hold as in cases (a) and (b).

Subcase (ii) $u_{0} \leq\left|-\nu+\psi_{y}^{T} C \theta_{0 y}+\psi_{u_{c}}^{T} \theta_{0 u}+\psi_{\nu}^{T} \theta_{0 u}\right|$.

Then

$$
\begin{aligned}
z(t) & =\sqrt{n_{d}}\left|\beta_{0}\right|\left[\left|-\nu+\psi_{y}^{T} C \theta_{0 y}+\psi_{u_{c}}^{T} \theta_{0 u}+\psi_{\nu}^{T} \theta_{0 u}\right|-u_{0}\right] \\
& \leq\left\|\psi_{y}(t)\right\| \text { if } \psi_{y}(t) \in \mathcal{A}
\end{aligned}
$$

since $\left\|\psi_{y}(t)\right\| \leq \psi_{\max }$. If $\left\|\psi_{y}(t)\right\| \leq \psi_{\min }$, direct substitution shows that $z(t) \leq \psi_{\min }$ and hence Eq. (B.18) holds, which proves (I) and (II).

Cases (a), (b), and (c) are mutually exclusive and collectively exhaustive and hence, the proof of Theorem 4.3 is completed. 


\section{Bibliography}

[1] K.S. Narendra and A.M. Annaswamy. Stable Adaptive Systems. Prentice Hall, Englewood Cliffs, N.J., 1989.

[2] K.J. Åström and B. Wittenmark. Adaptive Control. Addison Wesley, New York, 1988.

[3] G.C. Goodwin and K.S. Sin. Adaptive Filtering, Prediction, and Control. Prentice-Hall, 1984.

[4] M. Vidyasagar. "Nonlinear Systems Analysis". Prentice-Hall, New Jersey, 1993.

[5] Dragoslav D. Šiljak. "Nonlinear Systems". John Wiley \& Sons, Inc, New York, 1969.

[6] M.A. Aizerman and F.R. Gantmacher. Absolute Stability of Regulator Systems. HoldenDay, Inc., 1964.

[7] K.S. Narendra and R.M. Goldwin. "A geometric criterion for the stability of certain nonlinear nonautonomous systems". IEEE Transactions on Circuit Theory, CT11:406-408, 1964.

[8] G. Zames. "On the input-output stability of time-varying nonlinear feedback systems, part i: Conditions derived using concepts of loop gain, conicity and positivity". IEEE Transactions on Automatic Control, 11:228-238, 1966.

[9] G. Zames. "On the input-output stability of time-varying nonlinear feedback systems, part ii: Conditions involving circles in the frequency plane and sector nonlinearities". IEEE Transactions on Automatic Control, 11:465-476, 1966.

[10] K.S. Narendra and J.H. Taylor. Frequency Domain Criteria for Absolute Stability. Academic Press, New York, 1973. 
[11] R.V. Monopoli. "Adaptive control for systems with hard saturation". In IEEE Conference on Decision and Control, pages 841-843, Houston, Tx, 1975.

[12] I. Horowitz. "Feedback systems with rate and amplitude limiting". International Journal of Control, 40:1215-1229, 1984.

[13] G. Feng M.Palanswami and Y. Zhu. "Stability of rate constrained robust pole placement adaptive control systems". Systems \& Control Letters, 19:99-107, 1992.

[14] R.S. Smith J.C. Doyle and D.F. Enns. "Control of plants with input saturation nonlinearities". In Proceedings of the 1987 ACC, pages 1034-1039, Minnesota, 1987.

[15] I. Horowitz. "A synthesis theory for a class of saturation systems". International Journal of Control, 38:169-187, 1983.

[16] A.H. Glattfelder and W. Schauffelberger. "Stability analysis of single loop control systems with saturation and antireset-windup circuits". IEEE Transactions on Automatic Control, AC-28, December 1983.

[17] Petros. Kapasouris. "Design for Performance enhancement in Feedback Control Systems with Multiple Saturating Nonlinearites". $\mathrm{PhD}$ thesis, Massachusetts Institute of Technology, 1988.

[18] Per-Olof Gutman and Per Hagander. "A new design of constrained controllers for linear systems". IEEE Transactions on Automatic Control, AC-30:22-33, January 1985.

[19] Von J.S. Karmakar and Dragoslav D. Šiljak. "Maximization of absolute stability regions by mathematical programming methods". Regelungstechnik, 1975.

[20] J.A. Walker and N.H. McClamroch. "Finite regions of attractions for the problem of luré". International Journal of Control, 6:331-336, 1967.

[21] A.R. Teel. "Global stabilization and restricted tracking for multiple integrators with bounded controls". Systems \& Control Letters, 18:165-171, 1992.

[22] H. J. Sussmann and Y. Yang. "On the stabilizability of multiple integrators by means of bounded feedback controls". In Proceedings of the 30th IEEE Conference on Decision and Control, Brighton, England, December 1991. 
[23] F. Ohkawa and Y. Yonezawa. "A discrete model reference adaptive control system for a plant with input amplitude constraints". International Journal of Control, 36:747-753, 1982.

[24] A.N. Payne. "Adaptive one-step-ahead control subject to an input-amplitude constraint". International Journal of Control, 43:1257-1269, 1986.

[25] C. Zhang and R.J. Evans. "Amplitude constrained adaptive control". International Journal of Control, 46:53-64, 1987.

[26] C. Zhang and R.J. Evans. "Amplitude constrained direct self-tuning control". In IFAC Symposium on Identification and System Parameter Estimation, pages 325-329, Beijing, China, 1988.

[27] C. Zhang and R.J. Evans. "Adaptive pole-assignment subject to saturation constraints". International Journal of Control, 46:1391-1398, 1987.

[28] D.Y. Abramovitch and G.F. Franklin. "On the stability of adaptive pole-placement controllers with a saturating actuator". IEEE Transactions on Automatic Control, 35:303-306, 1990.

[29] C. Zhang G. Feng and M. Palaniswamy. "Stability analysis of input constrained continuous time indirect adaptive control". Systems \& Control Letters, 17:209-215, 1991.

[30] C. Zhang. "Discrete time saturation constrained adaptive pole assignment control". IEEE Transactions on Automatic Control, (to appear), 1993.

[31] H. Wang and J. Sun. "Modified model reference adaptive control with saturated inputs". In Proceedings of the 31st Conference on Decision and Control, Tucson, Arizona, 1992.

[32] Gene F. Franklin J. David Powell and Michael L. Workman. "Digital Control of Dynamic Systems". Addison-Wesley, Inc., 1990.

[33] A.M. Annaswamy and M.A. Srinivasan. The computational theory of haptics. Private Communication, 1990. 
[34] A.M. Annaswamy and M.A. Srinivasan. "Identification for grasping and manipulation of compliant objects with compliant fingerpads". Submitted to the IEEE Transactions on Automatic Control, 1991. 